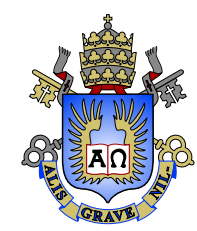

Jéferson Rômulo Pereira Coêlho

\title{
Uma Solução Eficiente para Subdivisão de Malhas Triangulares
}

Dissertação apresentada como requisito parcial para obtenção do grau de Mestre pelo Programa de Pósgraduação em Informática do Departamento de Informática da PUC-Rio

Orientador: Prof. Marcelo Gattass 


\title{
Jéferson Rômulo Pereira Coêlho
}

\section{Uma Solução Eficiente para Subdivisão de Malhas Triangulares}

\begin{abstract}
Dissertação apresentada como requisito parcial para obtenção do grau de Mestre pelo Programa de Pósgraduação em Informática do Departamento de Informática do Centro Técnico Científico da PUC-Rio. Aprovada pela Comissão Examinadora abaixo assinada.
\end{abstract}

Prof. Marcelo Gattass

Orientador

Departamento de Informática - PUC-Rio

Prof. Hélio Côrtes Vieira Lopes

Departamento de Informática - PUC-Rio

Prof. Luiz Fernando Martha Departamento de Engenharia Civil - PUC-Rio

Dr. Pedro Mário Cruz e Silva Instituto Tecgraf/PUC-Rio

Prof. Waldemar Celes

Departamento de Informática - PUC-Rio

Prof. José Eugenio Leal Coordenador Setorial do Centro Técnico Científico -

PUC-Rio

Rio de Janeiro, 26 de Março de 2013 
Todos os direitos reservados. É proibida a reprodução total ou parcial do trabalho sem autorização da universidade, do autor e do orientador.

\section{Jéferson Rômulo Pereira Coêlho}

Graduou-se em Bacharel em Ciência da Computação pela Universidade Federal de Viçosa em janeiro de 2011.

Ficha Catalográfica

Coêlho, Jéferson Rômulo Pereira

Uma Solução Eficiente para Subdivisão de Malhas Triangulares / Jéferson Rômulo Pereira Coêlho; orientador: Prof. Marcelo Gattass. - Rio de Janeiro : PUC-Rio, Departamento de Informática, 2013.

v., 92 f: il. ; 29,7 cm

1. Dissertação (mestrado) - Pontifícia Universidade Católica do Rio de Janeiro, Departamento de Informática.

Inclui referências bibliográficas.

1. Informática - Tese. 2. Subdivisão de Malhas Triangulares. 3. Estruturas de Dados Topológicas. 4. Subdivisão Global. 5. Subdivisão Local. I. Gattass, Marcelo. II. Pontifícia Universidade Católica do Rio de Janeiro. Departamento de Informática. III. Título. 


\section{Agradecimentos}

A Deus por ter me dado força para suportar e superar todas as dificuldades.

Ao meu orientador, professor Marcelo Gattass, pelo apoio, atenção, incentivo e amizade durante toda a realização deste trabalho.

À minha familia, em especial aos meus pais Romilda Santos e Hélio Gilson os quais privei da minha companhia para me dedicar a este trabalho.

À Juliette Zanetti pelo companherismo e pelo apoio.

À todos os meus colegas do v3o2, em especial ao André Campos pelas ideias sempre interessantes e paciência.

À CAPES, a PUC-Rio e ao TECGRAF, pelo auxilios concedidos, sem os quais este trabalho não poderia ser realizado. 


\section{Resumo}

Coêlho, Jéferson Rômulo Pereira; Gattass, Marcelo. Uma Solução Eficiente para Subdivisão de Malhas Triangulares. Rio de Janeiro, 2013. 92p. Dissertação de Mestrado — Departamento de Informática, Pontifícia Universidade Católica do Rio de Janeiro.

Subdivisão de superfícies triangulares é um problema importante nas atividades de modelagem e animação. Ao deformar uma superfície a qualidade da triangulação pode ser bastante prejudicada na medida em que triângulos, antes equiláteros, se tornam alongados. Uma solução para este problema consiste em refinar a região deformada. As técnicas de refinamento requerem uma estrutura de dados topológica que seja eficiente em termos de memória e tempo de consulta, além de serem facilmente armazenadas em memória secundária. Esta dissertação propõe um framework baseado na estrutura Corner Table com suporte para subdivisão de malhas triangulares. O framework proposto foi implementado numa biblioteca $\mathrm{C}++$ de forma a dar suporte a um conjunto de testes que comprovam a eficiência pretendida.

\section{Palavras-chave}

Subdivisão de Malhas Triangulares; Estruturas de Dados Topológicas; Subdivisão Global; Subdivisão Local; 


\section{Abstract}

Coêlho, Jéferson Rômulo Pereira; Gattass, Marcelo (advisor). An efficient solution for Triangular Mesh Subdivision. Rio de Janeiro, 2013. 92p. MSc Dissertation - Departamento de Informática, Pontifícia Universidade Católica do Rio de Janeiro.

Subdivision of triangular surfaces is an important problem in modeling and animation activities. Deforming a surface can be greatly affected the quality of the triangulation when as equilateral triangles become elongated. One solution to this problem is to refine the deformed region. Refinement techniques require the support of topological data structure. These structures must be efficient in terms of memory and time. An additional requirement is that these structures must also be easily stored in secondary memory. This dissertation proposes a framework based on the Corner Table data structure with support for subdivision of triangular meshes. The proposed framework was implemented in a $\mathrm{C}++$ library. With this library this work presents a set of test results that demonstrate the desired efficiency.

\section{Keywords}

Subdivision of triangle meshes; Topological Data Structures; Global Subdivision; Local Subdivision; 


\section{Sumário}

$\begin{array}{lr}\text { Lista de figuras } & 8\end{array}$

$\begin{array}{lr}\text { Lista de tabelas } & 11\end{array}$

Lista de Algoritmos $\quad 12$

1 Introdução 14

1.1 Motivação 14

1.2 Contribuições desta dissertação 15

1.3 Estrutura da Dissertação 15

2 Métodos de subdivisão $\quad 17$

2.1 Subdivisão Global 17

2.2 Subdivisão Adaptativa 23

3 Estruturas de Dados Topológicas $\quad 30$

3.1 Corner Table 30

3.2 CHE - Compact Half-Edge 36

3.3 Corner Table vs CHE 41

4 Operadores na Corner Table $\mathbf{4 2}$

4.1 Operadores Estelares para Malhas Triangulares 42

4.2 Edge Flip 43

4.3 Edge Flip Inverso $\quad 45$

4.4 Edge Split $\quad 46$

4.5 Edge Weld 48

5 Subdivisão em Corner Table 51

5.1 Composição de operações 51

5.2 Subdivisão Global 53

5.3 Subdivisão Local $\quad 58$

$\begin{array}{ll}5.4 & \text { Undo e Redo para Subdivisão }\end{array}$

6 Resultados $\quad 71$

6.1 Subdivisão Global

6.2 Subdivisão adaptativa 80

7 Conclusão e Trabalhos Futuros $\quad 89$

Referências Bibliográficas $\quad 90$

Referências Bibliográficas $\quad 90$ 


\section{Lista de figuras}

1.1 Interpretação de superfícies a partir de um plano de corte 15

2.1 Subdivisão 1 para 4

2.2 Máscara para posicionamento dos vértices pares e ímpares em Loop.

2.3 Três níveis de subdivisão Loop. As arestas em destaque mostram o refinamento 1 para 4 . Fonte: (Pakdel and Samavati, 2007)

2.4 Máscara para posicionamentos dos novos vértices para o caso de malhas regulares em Butterfly.

2.5 Máscara para posicionamentos dos novos vértices para o caso de malhas regulares em Butterfly considerando $w=0$.

2.6 Máscara para posicionamento dos vértices ímpares para o caso em que a valência de $\mathbf{v}^{i}$ é três.

2.7 Máscara para posicionamento dos vértices ímpares para o caso em que a valência de $\mathbf{v}^{i}$ é quatro.

2.8 Máscara para posicionamento dos vértices ímpares para valências de $\mathbf{v}^{i}$ diferentes de três e quatro.

2.9 Suavização usando Butterfly modificado. Fonte: (Zorin et al, 1996)

2.10 Fissura gerada pela simples subdivisão de uma face e corrigida por uma triangulação simples.

2.11 Uso de triangulação simples para remover fissuras. A partir do segundo passo de subdivisão os vértices não são reposicionados de forma correta

2.12 Triangulação usando red-green e malha restrita. Fonte: (Pakdel and Samavati, 2007)

2.13 Expansão $E^{1}(S), E^{2}(S)$ e $E^{3}(S)$ dos vértices selecionados. Os números mostram a distância do vértice à área selecionada

2.14 Dois passos de subdivisão aplicados sobre a mesma região com expansão $E^{1}(S)$. As fissuras foram resolvidas usando a triangulação simples, ou seja, conectando os vértices $T$ aos vértices $O$. A área em destaque identifica a região que deve ser dividida a cada passo e os vértices em vermelho pertencem a $S^{\prime}$.

2.15 Exemplo da aplicação da subdivisão incremental em uma malha de controle usando Loop e Butterfly. Fonte: (Pakdel and Samavati, 2007)

3.1 Relação entre corner e half-edge. Adaptado de: (Ferreira, 2006)

3.2 Representação de uma malha de triângulos usando Corner Table. Adaptado de (Vieira, 2003) 
3.3 Corners $n(c), p(c), l(c)$ e $r(c)$ que resultam das operações de next, previous, left e right, respectivamente. O índice do triângulo ao qual pertence o corner $c$, é denotado por $t(c)$. Adaptado de (Rossignac et al, 2001) e (Vieira, 2003) 32

3.4 Representação incluindo a tabela $C$

3.5 A estrela de um vértice e a representação implícita de arestas 34

3.6 Vizinhança de uma aresta 36

3.7 Nível 0 da CHE: Sopa de triângulos. Adaptado de (Ferreira, 2006) 37

3.8 Relação entre vértice e half-edge. Adaptado de (Ferreira, 2006) 38

3.9 Nível 1 da CHE: Adjacência entre os triângulos. Adaptado de (Ferreira, 2006)

3.10 Half-edges opostas. Adaptado de (Ferreira, 2006)

3.11 Escolha das half-edges para serem armazenadas na tabela $V H$. Adaptado de (Ferreira, 2006)

4.1 Operações estelares de subdivisão. Fonte: (Velho, 2003) 42

4.2 Edge Flip como uma composição de edge split + edge weld 43

4.3 Edge Flip na Corner Table

4.4 A aplicação do Edge Flip faz com que as faces girem no sentido anti-horário

4.5 Edge Flip inverso

4.6 Edge Split na Corner Table

4.7 Convenção para posicionamento das faces após a realização da operação de Edge Split

4.8 Edge Weld na Corner Table

5.1 Subdivisão 1 para 4

5.2 Subdivisão 1 para 4 como uma sequência de 3Edge Split + Edge Flip

5.3 Subdivisão 1 para 4 subdividindo faces adjacentes

5.4 a) Malha inicial a ser subdividida. b-e) Os pares de cornes $(v, e)$ são retirados da fila e as faces em suas estrelas que ainda não foram processadas são subdivididas. Ao processar um vértice, as faces de sua estrela são marcadas como processadas e as faces opostas são marcadas como visitadas. Após subividir toda estrela do vértice, o operador de Edge Flip é aplicado sobre a aresta oposta ao corner e. f) Malha refinada. 
5.5 a) Malha inicial. b) $O$ operador de Edge Split é inicialmente aplicado a partir do corner $c$. O par $(v, e)$ é colocado na fila Q. c) Ao subdvidir as faces na estrela do vértice $V[v]$, o operador de Edge Split é aplicado a partir do corner $t$ e o par $(e, g)$ é colocado na fila Q. d) Após processar o vértice $V[v]$, o operador de Edge Flip é aplicado a patir do corner $e$. Apenas o corner $t=\operatorname{prev}(e)$ associado ao vértice que já foi processado muda de vértice, logo os outros corners da face que podem já está na fila associados com os seus vértices continuam associados com os mesmos vértices.

5.6 Exemplo da aplicação do algoritmo de subdivisão em um triângulo usando Corner Table. Sequência de aplicação dos operadores: EdgeSplit( 0 ), EdgeSplit( 1 ), EdgeSplit( 5 ) e EdgeFlip (2)

5.7 Ilustração do funcionamento do Algoritmo 18. As faces em azul são aquelas que serão subdivididas posteriormente.

5.8 Inversão do operador de Edge Split pela aplicação do operador de Edge Weld e vice-versa.

5.9 Invrsão do operador de Edge Flip pela aplicação do Edge Flip Inverso e vice-versa.

6.1 Geração da malha de uma esfera através da subdivisão de um icosaedro regular

6.2 Subdivisão de uma malha com dois triângulos para aproximar a função $z=\sin (\pi x) \cos y+0.1 \sin (2 \pi x)$

6.3 Subdivisão de uma um tetraedro regular usando Butterfly $\quad 75$

6.4 Subdivisão de uma estrela de 6 pontas usando Butterfly $\quad 76$

6.5 Subdivisão do modelo cow usando Butterfly. 77

6.6 Quatro níveis de subdivisão do modelo bunny usando Butterfly. 78

6.7 Subdivisão do modelo bimba usando Butterfly. O triângulo muito fino na em 6.7 (a) provoca um efeito indesejado na subdivisão

6.8 Vários níveis da subdivisão incremental aplicada sobre um icosaedro regular para geração da malha de uma esfera.

6.9 Subdivisão adaptativa de uma malha inicial com 8 triângulos para aproximar a função $z=\sin (\pi x) \cos y+0.1 \sin (2 \pi x)$. A cada passo a região onde o ponto médio de uma aresta dista mais que 0.005 da posição onde deveria está é selecionada para subdivisão

6.10 Vários níveis da subdivisão incremental aplicada sobre um icosaedro regular para geração da malha de uma esfera.

6.11 Subdivisão incremental do modelo cow usando Butterfly para posicionar os novos vértices criados. Os pontos em destaque pertecem ao conjunto $S^{\prime}$, ou seja, o pontos a partir de onde o algoritmo faz a expansão.

6.12 Subdivisão incremental do modelo bunny usando Butterfly para posicionar os novos vértices criados.

6.13 Subdivisão incremental do modelo bimba usando Butterfly para posicionar os novos vértices criados. 


\section{Lista de tabelas}

$\begin{array}{lll}5.1 & \text { Operações Inversas } & 70\end{array}$

6.1 Tempo para realizar a subdivisão global. *Core i5 3.10Ghz, 8GB RAM

6.2 Dados da realização da subdivisão adaptativa com $r=1$. ${ }^{*}$ Core i5 3.10Ghz, 8GB RAM

6.3 Dados da realização da subdivisão adaptativa com $r=1$. ${ }^{*}$ Core i5 3.10Ghz, 8GB RAM

6.4 Dados da realização da subdivisão adaptativa com $r=5$. *Core i5 3.10Ghz, 8GB RAM

6.5 Dados da realização da subdivisão adaptativa com $r=5$. ${ }^{*}$ Core i5 3.10Ghz, 8GB RAM 


\section{Lista de Algoritmos}

1 edge( $\mathrm{c}$ ) 34

2 VizinhançaDeUmvértice $(v)$. Fonte: (Vieira, 2003) 35

3 VizinhançaDeUmaAresta( $e$ ). Fonte: (Vieira, 2003) 35

4 VizinhançaDeUmaFace $(f)$. Fonte: (Vieira, 2003) 36

5 Percorrimento das curvas de bordo( ). Fonte: (Ferreira, 2006) 40

6 EdgeFlip $\left(c_{0}\right)$. Adaptado de (Vieira, 2003) 44

7 EdgeFlipInverso $\left(c_{1}\right) \quad 46$

8 EdgeSplit $\left(c_{0}\right) \quad 48$

9 EdgeWeld $\left(c_{1}\right)$. Adaptado de (Vieira, 2003) 49

10 SubdivisaoGlobal $\quad 55$

11 runSplit $\quad 56$

12 Subdivisaolncremental 58

13 obtemCornersDosVertices $\quad 59$

14 obtemCornersDosVertices 60

15 obtemCornersNaVizinhancaR 61

16 obtemVerticesVizinhos $\quad 62$

17 expandeVertice $\quad 63$

18 obtemFacesFormadasPorErS 64

19 runSplit 66

20 SubdivideFaces $\quad 67$ 
Quando se culpa os outros, renuncia-se à capacidade de mudar.

Douglas Adams, . 


\section{Introdução}

Métodos de subdivisão são cada vez mais utilizados em pacotes de animação e modelagem computacional, substituindo ferramantes tradicionais de modelagem, como NURBS. As operações de subdivisão são eficientes e permitem a construção de superfícies que tem suavidade variável, além ferramentas de modelagem intuitivas que podem ser aplicadas em uma malha de topologia arbitrária. No entanto, em muitos casos, a subdivisão é realizada tendo como suporte uma estrutura de dados topológica que consome um grande volume de memória, como uma Half$E d g e$, tornando difícil a sua aplicação em modelos massivos.

\section{1 Motivação}

A motivação para realizar subdivisão local em Corner Table surgiu do problema de iterativamente ajustar superfícies a dados sísmicos. Seguindo uma prática comum no processo de interpretação sísmica, a superfície, que geralmente representa um horizonte, uma falha ou um sal, é posicionada no volume a partir de uma manipulação direta de suas curvas de interseção com um plano de corte qualquer (ver Figura 1.1).

À medida que o usuário vai deformando a superfície, a malha triangular inicial precisa localmente ser simplificada ou refinada para se adaptar à geometria e manter a qualidade dos triângulos.

O refinamento e a simplificação podem ser baseados na curvatura da superfície ou mesmo na área dos triângulos, ou seja, se um triângulo atingir uma determinada área limite ele é refinado ou se sua área se tornar muito pequena ele é simplificado.

Uma vez que dados sísmicos usam grandes volumes de memória, é desejável que a interpretação de superfícies faça uso eficiente da memória. Por este motivo, a estrutura de dados topológica escolhida para representação da superfície foi a Corner Table. Esta estrutura é simples e usa apenas um vetor de inteiros da dimensão da lista de triângulos para representar a topologia da superfície. 
Por se tratar de uma aplicação de modelagem interativa, também é desejável fornecer algum mecanismo de undo/redo para que o usuário possa desfazer ou refazer uma sequência de passos durante o processo.

(Vieira et al, 2004) propuseram a simplificação de malhas triangulares baseadas em Corner Table, o autor entretando não encontrou na literatura trabalhos com subdivisão e suporte para undo/redo em Corner Table. Desta forma, o objetivo deste trabalho é realizar subdivisão global e local de forma eficiente usando a Corner Table como estrutura de dados topológica de suporte, além de fornecer uma forma para implementação do mecanismo de undo/redo que preserve a relação biunivica entre a triangulação e a estrutura de dados que a implementa, ou seja, ao executar uma operação de undo, por exemplo, a estrutura de dados volta à mesma configuração que possuía anteriormente..
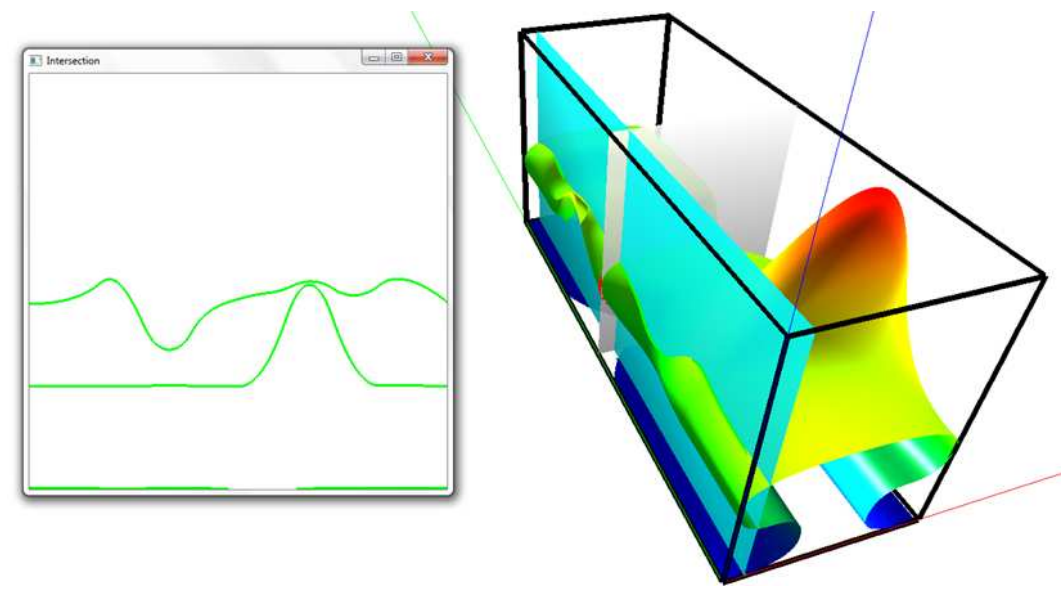

Figura 1.1: Interpretação de superfícies a partir de um plano de corte

\section{2 \\ Contribuições desta dissertação}

Como contribuição desta dissertação destacam-se:

1. Nova implementação dos operadores estelares inversíveis.

2. Dois algoritmos para realizar subdivisão global e local para malhas triangulares em Corner Table

\section{3}

\section{Estrutura da Dissertação}

Esta dissertação está dividida da seguinte forma: no Capítulo 2 apresentamos os esquemas de subdivisão global e local que são utilizados neste trabalho, no Capítulo 3 apresentamos duas estruturas de dados topológicas: a Corner Table e a Compact Half-Edge. Ambas são estruturas que utilizam um pequeno volume para 
representação de superfícies triangulares e poderiam ser utilizadas neste trabalho. Concluímos este Capítulo com a escolha da Corner Table por ser mais simples. No Capítulo 4 apresentamos uma nova implementação em Corner Table para um conjunto de operadores estelares inversíveis que servem de base para realizar a subdivisão e as suas operações inversas. No Capítulo 5 propomos dois novos algoritmos para realizar subdivisão global e local e o mecanismo de undo/redo baseados nestes operadores. No Capítulo 6 apresentamos os resultados e obtidos, e por fim, no capítulo 7, apresentamos algumas conclusões e propomos trabalhos futuros. 


\section{2 \\ Métodos de subdivisão}

Métodos de subdivisão de superfícies são cada vez mais usados em pacotes de animação e modelagem computacional (Pakdel and Samavati, 2007). A subdivisão é definida por operações simples, de refinamento e de médias, que são uniformemente aplicados em uma dada malha de controle. Repetidas aplicações destas operações produzem uma sequência de malhas que convergem para uma superfície que é suave em toda sua extensão (Cashman, 2012). Algumas regras especiais de subdivisão possibilitam a construção de superfícies que variam a sua suavidade. Além disso, as operações de subdivisão são eficientes e permitem a construção de ferramentas de modelagem intuitivas e podem ser aplicadas em malhas de polígonos com topologia arbitrária. Consequentemente, subdivisão tem substituido ferramentas de modelagem tradicionais, tais como NURBS, em aplicações de modelagem e sólidos de forma livre (DeRose et al, 1998).

Iniciando com uma malha de polígonos de entrada, cada passo da subdivisão refina suas faces e reposiona seus vértices através de combinações afim dos vértices próximos, produzindo uma superfície suave no limite (Zorin et al, 1996). À medida que repetidos passos de subdivisão são aplicados, o número de faces aumenta exponencialmente, o que pode aumentar a carga de computação rapidamente (Pakdel and Samavati, 2007). No entanto, em muitas aplicações não é necessário, e muitas vezes nem desejável, que toda a malha seja refinada. Em visualização de modelos 3D, por exemplo, apenas as regiões visíveis da malha devem ser detalhadas, ou em outro caso, projetistas muitas vezes precisam focar apenas na área em que estão trabalhando.

Desta forma, as operações de subdivisão podem ser classificadas em dois tipos: subdivisão global e subdivisão adaptativa. No caso da subdivisão global, todas as faces da malha são subdivididas, ja na subdivisão adaptativa, apenas um conjunto das faces é subdividido.

\section{1}

\section{Subdivisão Global}

Muitos métodos de subdivisão de superfícies foram desenvolvidos nas últimas décadas, como Doo-Sabin (Doo and Sabin, 1978), Catmull-Clark 
(Catmull and Clark, 1998), Loop (Loop, 1987) e Butterfly (Dyn et al, 1990). Nós focaremos nos métodos de subdivisão Loop e Butterfly, pois, como citado em (Pakdel and Samavati, 2007), estes esquemas operam em malhas de triângulos, e são comumente utilizados em modelagem, renderização e sistemas de animação. Esses dois algoritmos compartilham a mesma operação de refinamento, mas os operadores de média, que definem as posições dos novos vértices, são diferentes. A Figura 2.1 mostra a operação de refinamento nos esquemas Loop e Butterfly. Em cada passo de subdivisão, cada face é dividida em quatro novas faces.

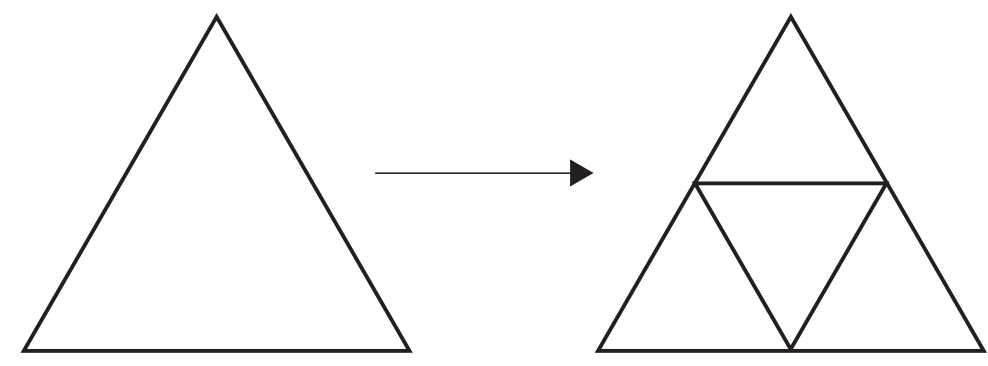

Figura 2.1: Subdivisão 1 para 4

Esta operação de refinamento tem duas características desejáveis: não altera a valência dos vértices pré-existentes na malha e todos os novos vértices criados tem valência 6 , com exceção daqueles que pertencem ao bordo da superfície.

Como destaca (Pakdel and Samavati, 2007), os esquema de subdivisão Loop produz uma superfície que é uma aproximação da malha de controle, uma vez que ele reposiciona os vértices da malha, já a superfície criada pelo esquema de subdivisão Butterfly interpola a malha de controle.

\subsection{1}

\section{Loop}

Começando com uma malha $M^{0}$, a malha $M^{i+1}$ é obtida através da divisão de todas as faces da malha $M^{i}$ e reposicionando seus vértices resultantes. A malha $M^{i}$ tem um nível de subdivisão $i$, além disso a resolução da malha e seu nível de subdivisão são diretamente relacionados, ou seja, a medida que o nível de subdivisão aumenta, as faces se tornam menores e a resolução da malha aumenta (Pakdel and Samavati, 2007).

No esquema de subdivisão Loop, proposto por (Loop, 1987), os operadores geométricos que definem as posições dos novos vértices são representados através de máscaras. Os vértices $\mathbf{v}^{i}$ da malha $M^{i}$ são reposicionados como uma combinação linear de sua posição com a dos seus vizinhos $\mathbf{v}_{j}^{i}$ de acordo com a equação ( 2-1 ).

$$
\mathbf{v}^{i+1}=\beta \mathbf{v}^{i}+\alpha \sum_{j=0}^{n-1} \mathbf{v}_{j}^{i}
$$

onde $n$ é a valência do vértice $\mathbf{v}^{i}$, 


$$
\begin{gathered}
\alpha=\frac{1}{n}\left(\frac{5}{8}-\left(\frac{3}{8}+\frac{1}{4} \cos \left(\frac{2 \pi}{n}\right)\right)^{2}\right) \\
\beta=1-n \alpha
\end{gathered}
$$

Os vértices $\mathbf{v}^{i+1}$ são chamados de vértices pares da malha $M^{i+1}$. A operação de divisão introduz vértices $\mathbf{e}^{i+1}$ sobre as arestas denominados vértices ímpares. Os vértices ímpares são calculados como na equação ( 2-4 )

$$
\mathbf{e}^{i+1}=\frac{3}{8} \mathbf{a}+\frac{3}{8} \mathbf{b}+\frac{1}{8} \mathbf{c}+\frac{1}{8} \mathbf{d}
$$

onde $\mathbf{a}, \mathbf{b}, \mathbf{c}$ e d são definidos como na Figura 2.2.

De acordo com (Pakdel and Samavati, 2007) a correta aplicação das equações ( 2-1) e ( 2-4 ) requerem que todos os vértices da malha $M^{i}$ esteja no mesmo nível de subdivisão. Isto é particularmente importante para subdivisão adaptativa.
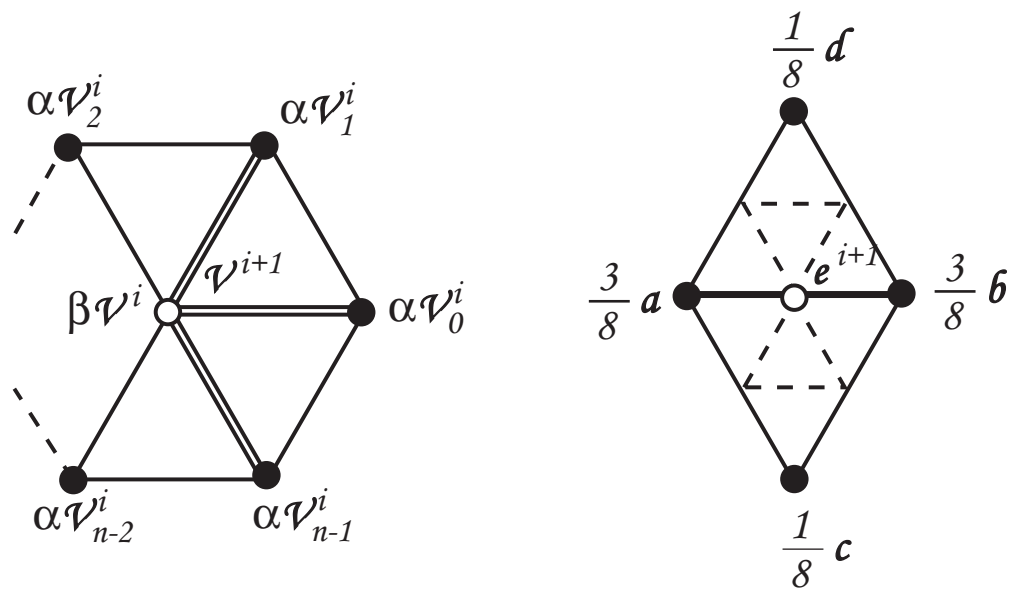

Figura 2.2: Máscara para posicionamento dos vértices pares e ímpares em Loop.

Em uma malha de triângulos, o esquema de subdivisão Loop tem continuidade paramétrica $C^{2}$ em todos os vértices de valência 6 , ditos vértices ordinários. Nos vértices extraordinários, aqueles os quais tem valência diferente de 6 , tem continuidade geométrica $G^{1}$ (Loop, 1987). Como o Loop usa a operação de refinamento 1 para 4 , os vértices ímpares são sempre ordinários enquanto os vértices pares mantém a sua valência.

\subsection{2}

\section{Butterfly}

O esquema de subdivisão Butterfly foi primeiro proposto por (Dyn et al, 1990). Ele é um esquema de interpolação, uma vez que não reposiciona os vértices da malha durante a subdivisão. Em suas primeiras versões, Butterfly era aplicável apenas em casos regulares, ou seja, casos em que os vértices eram ordinários. No caso regular, com os vértices $a$ e $b$ ordinários, de acordo com uma 


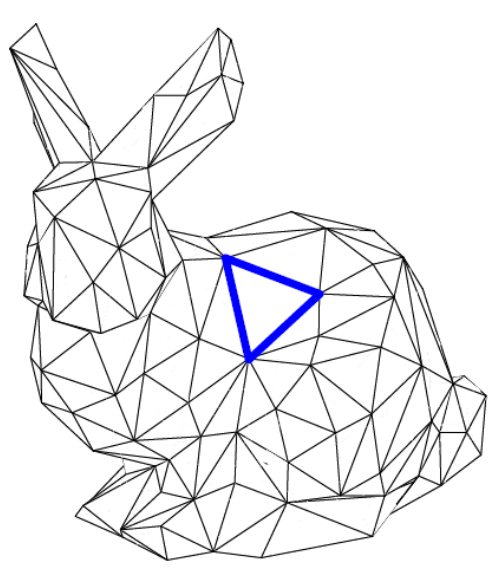

(a) 280 faces

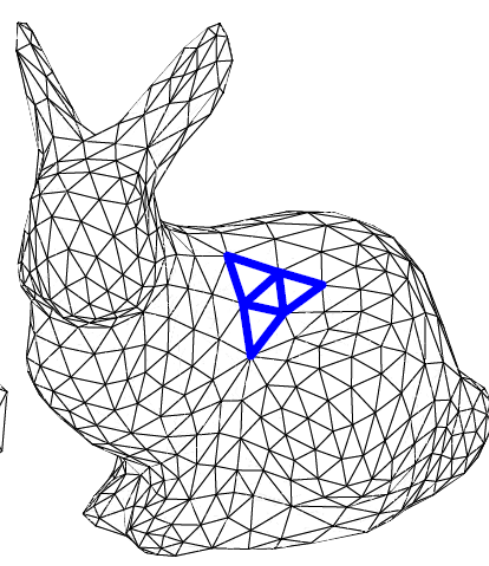

(b) 1120 faces

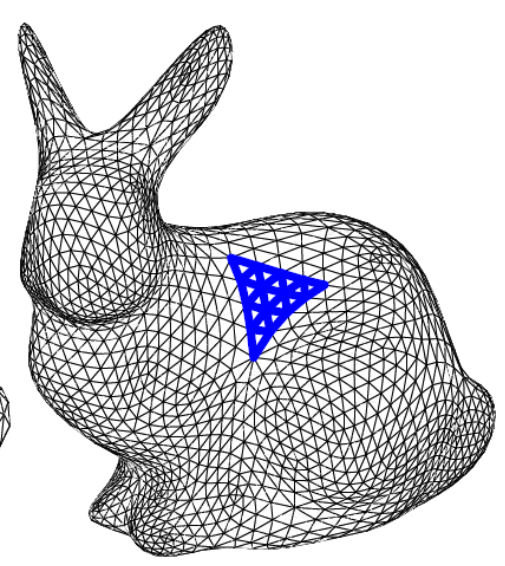

(c) 4480 faces

Figura 2.3: Três níveis de subdivisão Loop. As arestas em destaque mostram o refinamento 1 para 4. Fonte: (Pakdel and Samavati, 2007)

extensão do esquema Butterfly apresentada em (Dyn et al, 1993) a posição do novo vértice ímpar $\mathbf{e}^{i+1}$ sobre a aresta $\mathbf{a b}$ é dada por

$$
\mathbf{e}^{i+1}=\left(\frac{1}{2}-w\right)(\mathbf{a}+\mathbf{b})+\left(\frac{1}{8}+2 w\right)(\mathbf{c}+\mathbf{d})-\left(\frac{1}{16}-w\right)(\mathbf{e}+\mathbf{f}+\mathbf{g}+\mathbf{h})+w(\mathbf{i}+\mathbf{j})
$$

onde $\mathbf{a}, \mathbf{b}, \mathbf{c}, \mathbf{d}, \mathbf{e}, \mathbf{f}, \mathbf{g}, \mathbf{h}, \mathbf{i}$ e j são definidos como na Figura 2.4 e $w$ é um valor que deve ser escolhido de forma que seja muito pequeno. Como em (Zorin et al, 1996) e (Pakdel and Samavati, 2007) escolhemos $w=0$, simplificando o cálculo da posição do novo vértice $\mathbf{e}^{i+1}$ para

$$
\mathbf{e}^{i+1}=\frac{1}{2} \mathbf{a}+\frac{1}{2} \mathbf{b}+\frac{1}{8} \mathbf{c}+\frac{1}{8} \mathbf{d}-\frac{1}{16} \mathbf{e}-\frac{1}{16} \mathbf{f}-\frac{1}{16} \mathbf{g}-\frac{1}{16} \mathbf{h}
$$

onde $\mathbf{a}, \mathbf{b}, \mathbf{c}, \mathbf{d}, \mathbf{e}, \mathbf{f}, \mathbf{g}$ e $\mathbf{h}$ são definidos como na Figura 2.5.

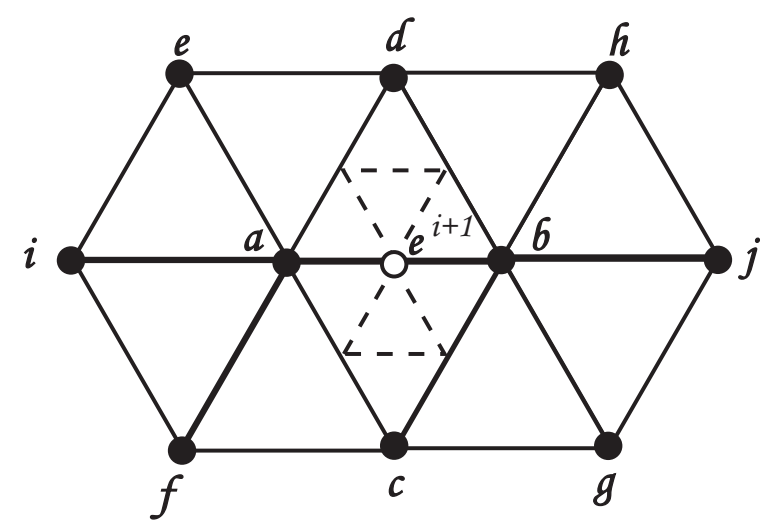

Figura 2.4: Máscara para posicionamentos dos novos vértices para o caso de malhas regulares em Butterfly.

Mais uma vez, para um resultado correto, todos os vértices envolvidos na computação de $\mathbf{e}^{i+1}$ devem está no mesmo nível de subdivisão.

Com o esquema original, as superfícies resultantes da subdivisão tem continuidade $C^{0}$ nos vértices extraordinários e $C^{1}$ nos demais. A fim de 


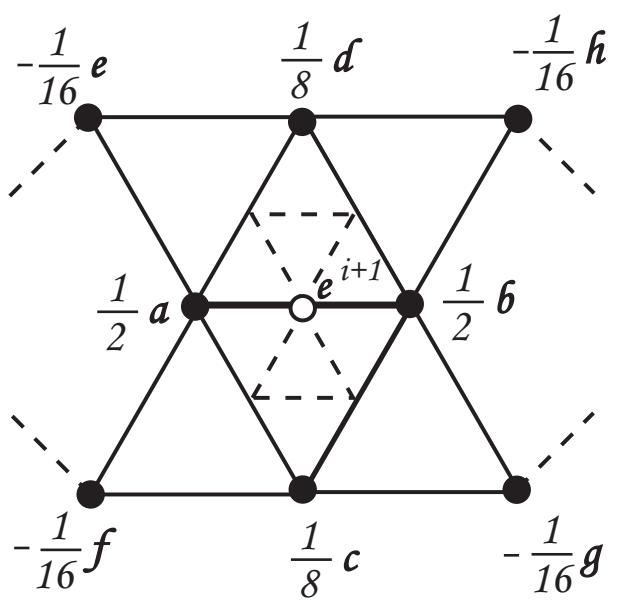

Figura 2.5: Máscara para posicionamentos dos novos vértices para o caso de malhas regulares em Butterfly considerando $w=0$.

garantir continuidade $C^{1}$ também em vértices extraordinários, (Zorin et al, 1996) modificaram a máscara original, de forma que, a posição do vértice ímpar $\mathbf{e}^{i+1}$ na $\operatorname{aresta} \mathbf{v}^{i} \mathbf{v}_{j}^{i}$ depende se um ou ambos os pontos extremos $\mathbf{v}^{i}$ e $\mathbf{v}_{j}^{i}$ são extraordinários. Este esquema ficou conhecido como Butterfly modificado.

No Butterfly modificado, se o vértice $\mathbf{v}^{i}$ é extraordinário e $\mathbf{v}_{j}^{i}$ é ordinário, a máscara de $\mathbf{e}^{i+1}$ é determinada de acordo com a valência $n$ de $\mathbf{v}^{i}$. Para valência três (ver Figura 2.6):

$$
\mathbf{e}^{i+1}=\frac{9}{12} \mathbf{v}^{i}+\frac{5}{12} \mathbf{v}_{j}^{i}-\frac{1}{12} \mathbf{v}_{j+1}^{i}-\frac{1}{12} \mathbf{v}_{j-1}^{i}
$$

Para valência quatro (ver Figura 2.7):

$$
\mathbf{e}^{i+1}=\frac{6}{8} \mathbf{v}^{i}+\frac{3}{8} \mathbf{v}_{j}^{i}-\frac{1}{8} \mathbf{v}_{j+2}^{i}
$$

E para todas as outras valências do vértice extraordinário vi (ver Figura 2.8):

$$
\mathbf{e}^{i+1}=\beta \mathbf{v}^{i}+\sum_{j=0}^{n-1} \alpha_{j} \mathbf{v}_{j}^{i}
$$

onde

$$
\alpha_{j}=\frac{1}{n}\left(\frac{1}{4}+\cos \frac{2 j \pi}{n}+\frac{1}{2} \cos \frac{4 j \pi}{n}\right)
$$

$\mathrm{e}$

$$
\beta=1-\sum_{j=0}^{n-1} \alpha_{j}
$$

Se ambos os vértices $\mathbf{v}^{i}$ e $\mathbf{v}_{j}^{i}$ são extraordinários, então o processo acima é repetido para cada vértice e o resultado final é dado pela média. 


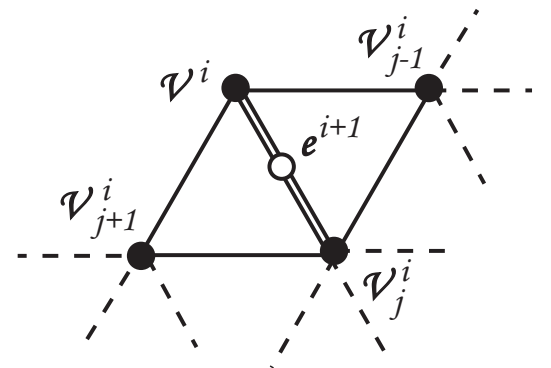

Figura 2.6: Máscara para posicionamento dos vértices ímpares para o caso em que a valência de $\mathbf{v}^{i}$ é três.

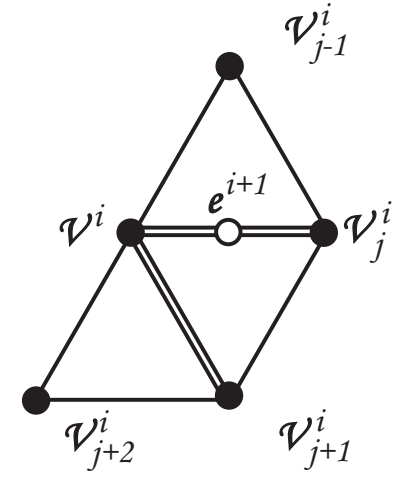

Figura 2.7: Máscara para posicionamento dos vértices ímpares para o caso em que a valência de $\mathbf{v}^{i}$ é quatro.

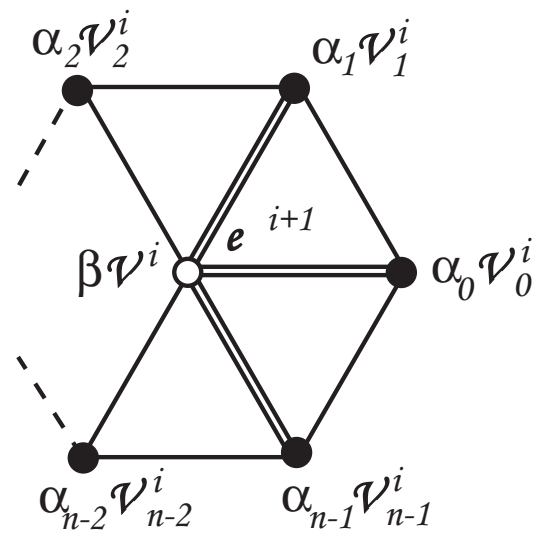

Figura 2.8: Máscara para posicionamento dos vértices ímpares para valências de $\mathbf{v}^{i}$ diferentes de três e quatro.
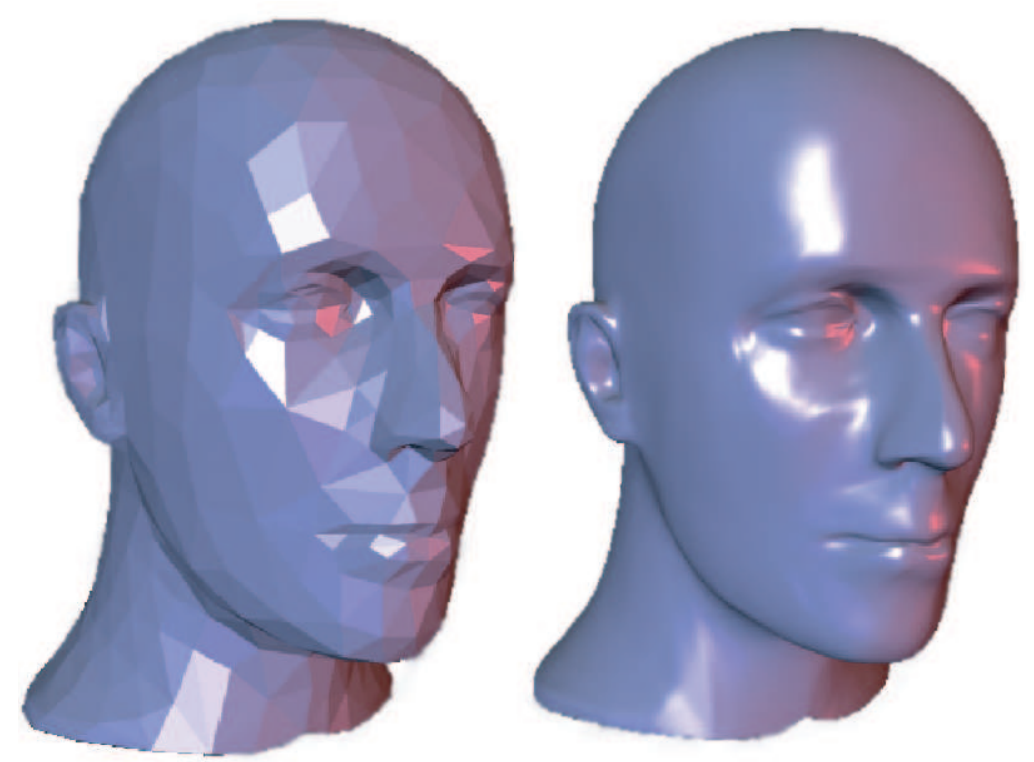

Figura 2.9: Suavização usando Butterfly modificado. Fonte: (Zorin et al, 1996) 


\section{2 \\ Subdivisão Adaptativa}

Como colocado por (Pakdel and Samavati, 2007), alguns esquemas de subdivisão são projetados de forma que o refinamento adaptativo é uma extensão natural do processo, como é o caso do $\sqrt{3}$-subdivision de (Kobblet, 2000) e o 4-8 subdivision de (Velho and Zorin, 2001). No entanto, pelas qualidades em relação às valências dos vértices da operação de refinamento 1 para 4 citadas na Seção 2.1, focaremos nos métodos que utilizam esse tipo de refinamento.

$\mathrm{Na}$ subdivisão adaptativa, apenas um subconjunto das faces da malha de controle e subdividida, no entanto, um cuidado maior deve ser tomado para lidar com a conectividade e as incossistências geométricas que podem surgir no processo. Como mostra a Figura 2.10, um simples passo para subdividir uma única face criou fissuras na malha, onde triângulos de níves de subdivisão diferentes se encontram em uma aresta. Uma malha 2-manifold é conforme se o resultado da interseção entre quaisquer duas faces é um vértice simples, uma aresta ou um conjunto vazio (Jones et al, 1997). Uma malha com fissuras não é conforme, desta forma, fissuras devem ser removidas para que a renderização, edição, animação e subdivisão da malha sejam feitas de maneira adequada (Pakdel and Samavati, 2007).

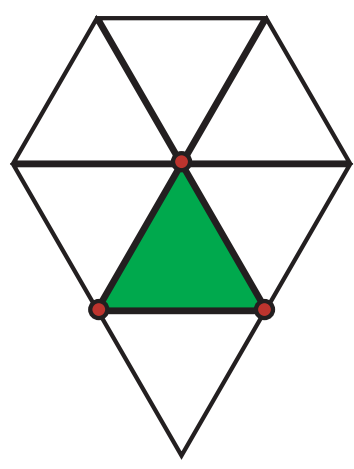

a) Face selecionada

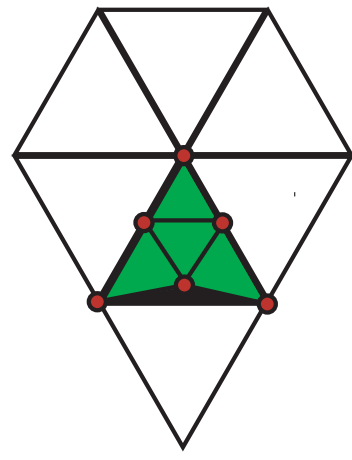

b) Fissura gerada

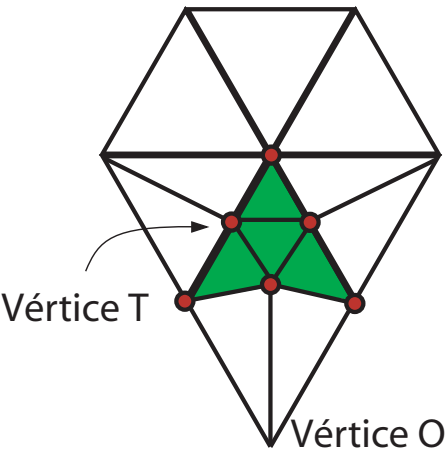

c) Triangulação para remover a fissura

Figura 2.10: Fissura gerada pela simples subdivisão de uma face e corrigida por uma triangulação simples.

De acordo com (Pakdel and Samavati, 2007), para remover as fissuras, é comum que as faces que as contém sejam trianguladas para produzir uma malha conforme. Essa triangulação muda a conectividade dos vértices dentro da área de subdivisão selecionada. Como alguns métodos, como Loop e Butterfly, usam os vizinhos para calcular a posição de um novo vértice, a mudança na conectividade dos vértices da área selecionada para subdivisão afeta o processo de subdivisão, e conduz a uma geometria inconsistente na superfície limite. Ainda de acordo com (Pakdel and Samavati, 2007), o ideal seria que a subdivisão adaptativa produzisse, na região selecionada da malha refinada, uma superfície limite igual à superfície 
gerada se toda a superfície fosse subdividida regularmente. Desta forma, todos os vértices dentro da região selecionada, deveriam ter a mesma concetividade como quando toda malha fosse subdividida.

Um outro efeito da subdivisão adaptativa é que repetidos refinamentos de uma região selecionada aumenta sua resolução exponencialmente em relação ao restante da malha. Em muitas aplicações, é desejável que a malha que foi adaptativamente subdividida seja balanceada, ou seja, que para quaisquer duas faces vizinhas, seus níveis de subdivisão não difiram em mais de uma unidade (Kobblet, 2000), de forma que o resultado seja um aumento gradual na resolução da regiões grosseiras para as regiões refinadas da malha.

Em (Pakdel and Samavati, 2007) são destacadas três propriedades que são desejáveis em uma subdivisão adaptativa:

1. conectividade consistente.

2. geometria consistente.

3. mudança gradual de resolução na superfície.

\subsection{1 \\ Manipulação das inconsistências}

Como mencionado anteriormente, a subdivisão de um subconjunto de faces da malha gera fissuras. Estas fissuras são devido à inserção de vértices ímpares em arestas compartilhadas por faces que estão em diferentes níveis de subdivisão. Para tratar tais inconsistências (Pakdel and Samavati, 2007) listam alguns algoritmos que trataremos de forma superficial abaixo.

Triangulação simples: Para resolver o problema das fissuras, (Amresh et al, 2002) propuseram um método de triangulação simples para dividir as faces vizinhas em duas, três ou quatro, dependendo do número de vértices ímpares. Para cada vértice ímpar, a divisão da face de menor nível de subdivisão remove a fissura. Os vértices ímpares são chamados de vértices $T$ e os vértices opostos que são conectados aos vértices $T$ são chamados de vértices $O$ (ver Figura 2.10).

O método de triângulação simples remove as fissuras da malha de forma bastante eficiente, porém, de acordo com (Pakdel and Samavati, 2007), ele apresenta alguns efeito colaterais indesejados. Primeiro, ele muda a conectividade e a valência dos vértices ímpares. Isto altera a superfície limite e ainda reduz a suavidade da superfície para $G^{1}$. Segundo, os vértices $O$ têm um nível diferentes de subdivisão em relação aos vértices pares da área selecionada, portanto, os vértices ímpares não serão corretamente reposicionados se a área selecionada for subdividida mais de uma vez (ver Figura 2.11). Por último, repetidas subdvisões e 
triangulações simples da área selecionada produzem uma alta valência dos vértices $O$, o que torna as faces alongadas.

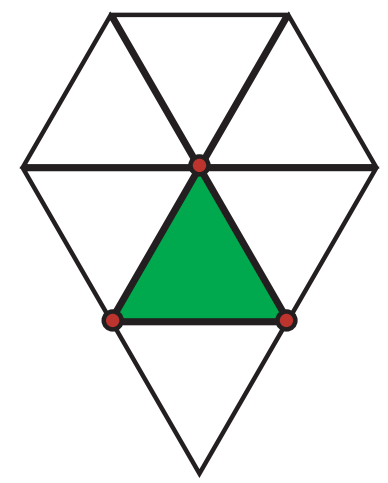

a) Face selecionada

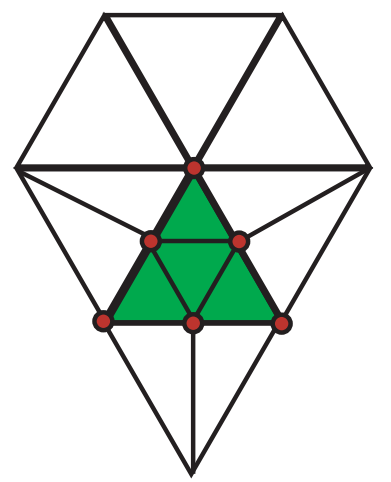

b) Primeira Subdivisão

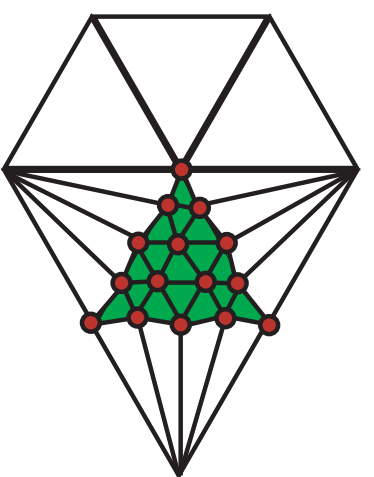

a) Segunda subdivisão

Figura 2.11: Uso de triangulação simples para remover fissuras. A partir do segundo passo de subdivisão os vértices não são reposicionados de forma correta

Triangulação red-green: outro método para remoção de fissuras pela inserção de vértices ímpares sobre arestas da malha é a triangulação red-green. Este método foi desenvolvido para permitir o refinamento adaptativo de malhas em análise de elementos finitos, onde uma malha é usada para aproximar equações em aplicações de análises numéricas.

A triangulação red-green garante que a conectividade da área selecionada não é afetada, além de evitar as altas valências dos vértices extraordinários e garantir que o nível de subdivisão entre quaisquer duas faces nunca difira de uma unidade, produzinho assim uma malha balanceada. No entanto, os vértices ímpares ainda têm vizinhos de diferentes níveis de subdivisão. Uma descrição mais detalhada da triangulação red-green pode ser encontrada em (Andrew et al, 1983).

Malha restrita: para evitar uma mudança na superfície gerada pela subdivisão adaptativa, vértices pares e ímpares devem estar no mesmo nível de subdivisão que seus vizinhos. (Zorin at al, 1997) definiram essa malha de malha restrita. Para obter uma malha restrita, faces são construídas de forma que os vértices da área selecionada tenham o mesmo nível de subdivisão. Uma descrição mais detalhada do algoritmo para obtenção de uma malha restrita pode ser encontrado em (Zorin at al, 1997).

Um algoritmo combinado: para uma solução que abranja as três propriedades desejáveis em uma subdivisão adaptativa colocadas anteriormente, (Pakdel and Samavati, 2007) cita um algoritmo que combina a triangulação redgreen com a malha restrita, uma vez que a triangulação reg-green resolve as questões de conectividade da subdivisão adaptativa e da mudança gradual entre os níveis de subdivisão e o algoritmo de malha restrita resolve a questão da computação correta dos vértices. No entanto, (Pakdel and Samavati, 2007) ressalta que este 
algoritmo não é eficiente e nem simples, uma vez que novos vértices $T$ podem ser gerados no processo e algoritmo deveria ser recursivamente aplicado até que não exista nenhum vértice $T$.

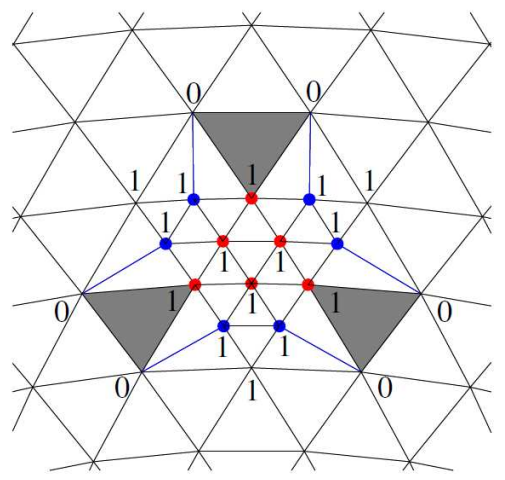

(a)Red-green

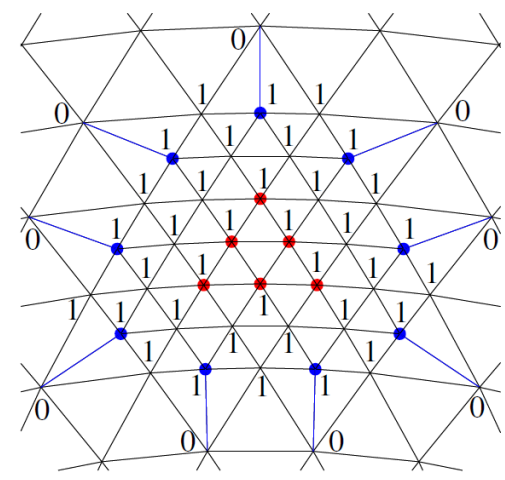

(b)Malha restrita

Figura 2.12: Triangulação usando red-green e malha restrita. Fonte: (Pakdel and Samavati, 2007)

\subsection{2}

\section{Subdivisão Incremental}

A subdivisão incremental, um esquema para subdivisão adaptativa proposto por (Pakdel and Samavati, 2007), satisfaz as três propriedades desejáveis para uma subdivisão adaptativa, ou seja, garante uma geometria e conectividade consistentes e a superfície resultante muda gradualmente sua resolução das áreas mais grosseiras para as áreas mais refinadas e, além disso, é um algoritmo eficiente e simples de implementar. Este algoritmo se propõe a realizar uma subdivisão adaptativa de forma que a área selecionada para a subdivisão tenha um resultado final como se a superfície fosse subdividida de forma global, além de resolver de forma eficiente as inconsistências geradas no processo de subdivisão adaptativa. Além disso, métodos para subdivisão global como Loop e Butterfly podem ser usados junto com a subdivisão incremental para realizar a subdivisão adaptativa. Por esses motivos, a subdivisão incremental foi o esquema escolhido pelo autor como método de subdivisão adaptativa.

Abaixo segue a descrição formal da subdivisão incremental. A descrição formal a seguir contém trechos retirados de (Pakdel and Samavati, 2007), onde o esquema é definido com mais detalhes.

\section{Descrição Formal}

Tome $V=\left\{\mathbf{v}_{0}, \mathbf{v}_{1}, \ldots, \mathbf{v}_{m-1}\right\}$ como sendo o conjunto de vértices da malha corrente. Tome $S$ como sendo um sub-conjunto de $V$. É desejável subdividir adaptativamente $S$ de forma que a superfície limite gerada a partir de $S$ seja 
exatamente a mesma como quando $V$ é subdividido. Para isso, o conjunto $S$ é expandido para um novo conjunto, maior que $S$, e então esse novo conjunto é subdividido.

Em cada nível de subdivisão, $S$ é expandido para $E^{r}(S)$ incluindo os vértices de $V$ que estão dentro de um raio $r$, em número de arestas, de pelo menos um vértice de $S$

$$
E^{r}(S)=\bigcup_{v \in S} N^{r}(\mathbf{v}), r>0
$$

onde $N^{r}(S)$ denota os vértices na vizinhança de $\mathbf{v}$ que distam $r$ arestas dele. Portanto, $w \in V$ está em $N^{r}(v)$ somente se existir uma caminho de $\mathbf{v}$ até $w$ com no máximo $r$ arestas ( ura 2.13). Após fazer a expansão, a subdivisão (Loop ou Butterfly por exemplo) deve ser realizada em $E^{r}(S)$ e as fissuras podem ser removidas eficientemente usando a triangulação simples (ver Figura 2.14). Ao final, tome $S^{\prime}$ como sendo a nova área selecionada (ver Figura 2.14), que é resultado da subdivisão adaptativa realizada em $S$.

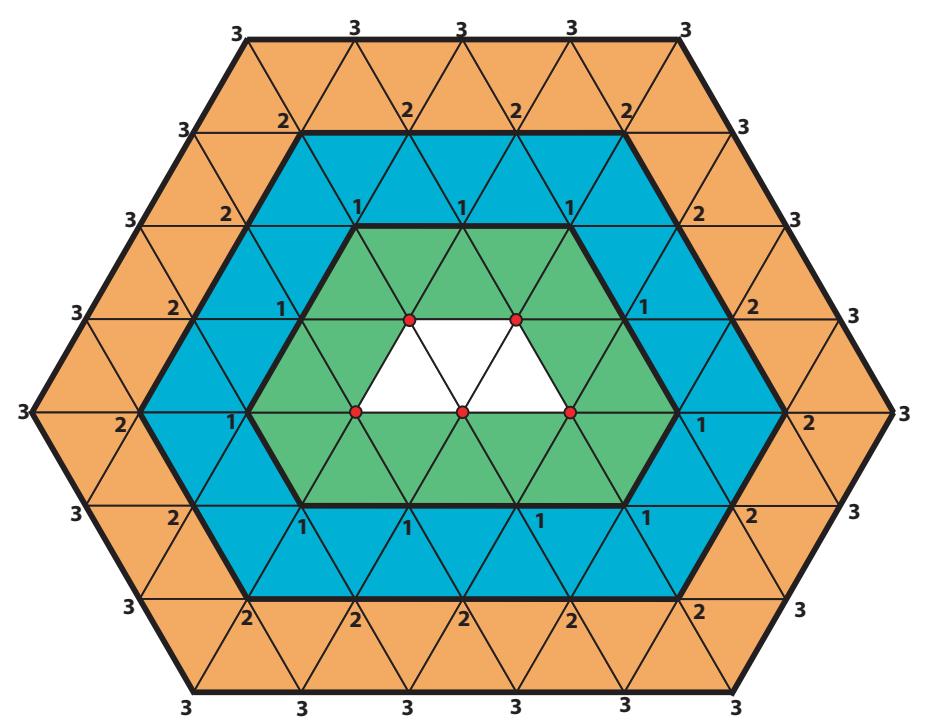

Figura 2.13: Expansão $E^{1}(S), E^{2}(S)$ e $E^{3}(S)$ dos vértices selecionados. Os números mostram a distância do vértice à área selecionada

(Pakdel and Samavati, 2007) destacam algumas características da subdivisão incremental. Estas características são listadas abaixo:

- A subdivisão adaptativa de $E^{r}(S)$ produz uma superfície limite a partir de $S$ que é exatamente a mesma como quando toda a malha é subdividida, uma vez que os vértices $O$ e $T$ ficam de fora do conjunto $E^{r}(S)$ (ver Figura 2.14), logo a conectividade dos vértices dentro de $S$ permanece inalterada. Além disso, os vértices de $S^{\prime}$ e seus vizinhos possuem o mesmo nível de subdivisão, uma vez que $E^{r}(S)$ inclui todos os vértices que tem uma distância, em arestas, menor 
ou igual a $r$ a partir de $S$ no processo de subdivisão. Com isso a superfície limite não é alterada devido à subdivisão adaptativa.
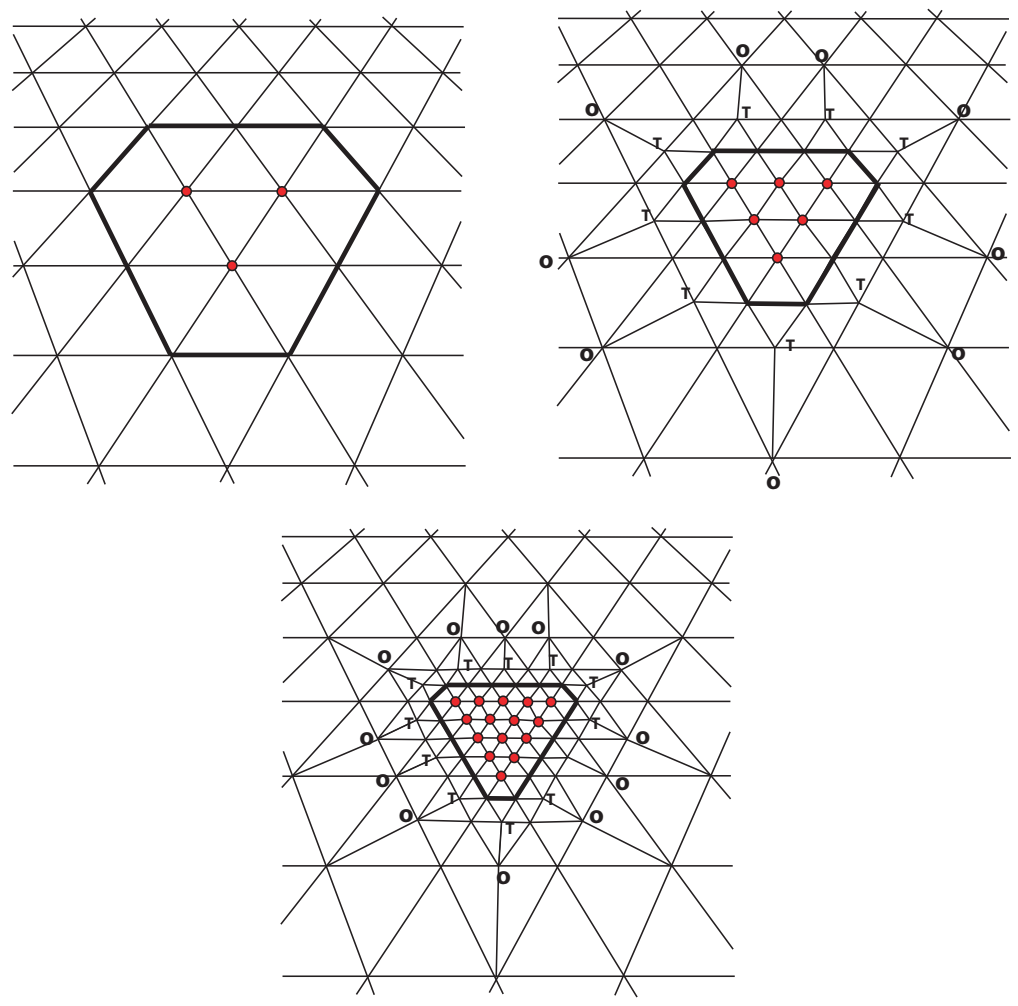

Figura 2.14: Dois passos de subdivisão aplicados sobre a mesma região com expansão $E^{1}(S)$. As fissuras foram resolvidas usando a triangulação simples, ou seja, conectando os vértices $T$ aos vértices $O$. A área em destaque identifica a região que deve ser dividida a cada passo e os vértices em vermelho pertencem a $S^{\prime}$.

- A subdivisão incremental mantém os vértices $O$ fora da área selecionada, uma vez que os vértices ímpares criados sobre as arestas que estavam a uma distânca $r$ do conjunto $S$ antes da subdivisão, passam a está a uma distância $2 r$ após a subdivisão. Os vértices $T$ da subdivisão adaptativa de $E^{r}(S)$ estão sempre a $2 r$ unidades de $S^{\prime}$ e os vértices $O$ sempre a $3 r$ unidades. Isto impede que a subdivisão incremental produza altas valências nos vértices $O$ (ver Figura 2.14).

- Na subdivisão incremental os vértices à uma distância $3 r$ de $S^{\prime}$ têm sempre um nível de subdivisão abaixo do que os vértices que estão a uma distância $2 r$, isso significa que a superfície é balanceada e que a superfície resultante tem um aumento gradual no nível de subdivisão das áreas mais grosseiras para as áreas que foram incrementalmente subdivididas.

- O parâmetro $r$ controla o grau de suavidade da transição entre os níveis de subdivisão. Um grande valor de $r$ incluirá um grande número de vizinhos na área selecionada o que permite uma transição suave das áreas mais grosseiras para as regiões mais refinadas. $\mathrm{O}$ aumento da área de transição é benéfico para 
os casos em que a região selecionada é subdividida muitas vezes. Na prática, poucos refinamentos são necessários para obter uma superfície suave e em muitos casos a expansão $E^{1}(S)$ é utilizada.

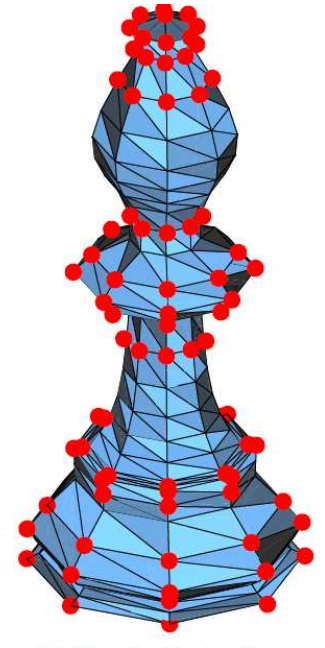

Malha de Controle

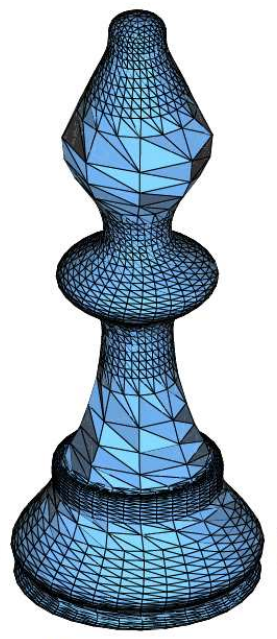

Loop

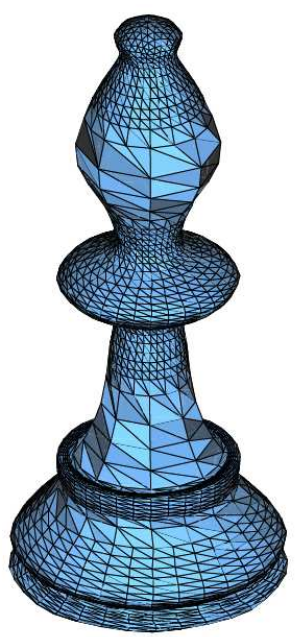

Butterfly

Figura 2.15: Exemplo da aplicação da subdivisão incremental em uma malha de controle usando Loop e Butterfly. Fonte: (Pakdel and Samavati, 2007) 


\section{3}

\section{Estruturas de Dados Topológicas}

Como destaca (Ferreira, 2006), a estrutura de dados Winged-Edge, proposta por (Baumgart, 1972), foi uma das primeiras estruturas para representação de superfícies em $\mathbb{R}^{3}$. Posteriormente essa estrutura sofreu muitas modificações a fim de aumentar a variedade de objetos a serem representados por ela. Entre essas modificações, destacam-se a inserção de laços (Braid et al, 1980) e a criação de uma nova estrutura de dados a partir dela chamada Half-Edge (Mantyla, 1987). Estas estruturas são muito poderosas no que diz respeito às suas capacidades de representação,uma vez que são capazes de representar subdivisões planares quaisquer, no entanto elas utilizam uma quantidade dispendiosa de memória, tornando-as muitas vezes inviáveis para aplicações com modelos muito grandes.

Mais recentemente, (Rossignac et al, 2001) e (Ferreira, 2006) propuseram duas estruturas de dados para malha triangulares muito concisas, Corner Table e $\mathrm{CHE}$ respectivamente. Ambas as estruturas se utilizam apenas de vetores de inteiros e um conjunto de regras para representar superfícies.

Neste capítulo, faremos uma revisão das estruturas de dados Corner Table e CHE, umas vez que estas duas estruturas são eficientes em termos de memória na representação de malhas triangulares e podem ser usadas em aplicações que envolvem grandes modelos.

\section{1}

\section{Corner Table}

A Corner Table é uma estrutura de dados topológica muito concisa que foi introduzida por (Rossignac et al, 2001) para ser usada em compressão de malhas triangulares. A estrutura foi usada para codificar e decodificar, em um formato compacto, malhas triangulares em um esquema chamado Edgebreaker.

A Corner Table foi apresentada de maneira bastante superfícial em (Rossignac et al, 2001), mas (Vieira, 2003) fez uma apresentação mais didática da mesma, e com alguns exemplos de algumas buscas topógicas básicas que facilitam a compreensão do funcionamento desta estrutura de dados, além de apresentar as implementações de algumas operações que serão utilizadas neste trabalho. Por estes motivos, optamos por apresentar a Corner Table da mesma forma, utilizando-se de 
alguns trechos e exemplos apresentados em (Vieira, 2003), que serão devidamente citados.

\subsection{1}

\section{Representação}

Como destaca (Ferreira, 2006), a Corner Table usa um conceito equivalente ao conceito de half-edge: o corner. Um corner é uma associação de uma face a um dos seus vértices, enquanto que a half-edge é uma associação de uma face à uma de suas arestas. As duas abordagens são equivalentes, uma vez que para cada corner, sempre existe uma half-edge oposta a ele (Figura 3.1).

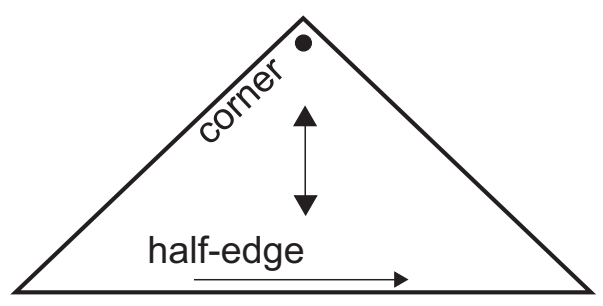

Figura 3.1: Relação entre corner e half-edge. Adaptado de: (Ferreira, 2006)

Na Corner Table, cada triângulo orientado é representado por 3 corners consecutivos que são indexados de acordo com a sua orientação. Assim uma superfície triangular formada por $n$ triângulos possuirá $3 n$ corners sendo, por definição, o triângulo orientado de índice 0 representado pelos cornes 0,1 e 2, o triângulo de índice 1 representado pelos corners 3, 4 e 5, de modo que o $i$-ésimo triângulo posssui os corners $3 i, 3 i+1$ e $3 i+2$. Desta forma, um corner cé associado ao triângulo de índice $t$ da seguinte forma:

$$
t(c)=\lfloor c \div 3\rfloor
$$

Como apresentado em (Vieira, 2003) e (Rossignac et al, 2001), a representação da topologia na Corner Table consiste em duas tabelas de inteiros, $O$ e $V$, com dimensões iguais a $3 n$, onde $n$ é o número de triângulos. Para cada corner c é gravado: $V[c]$, vértice do corner, e $O[c]$, corner oposto ao corner $c$. Os atributos dos vértices, juntamente com sua geometria, são armazenados em uma outra tabela $G$, cuja dimensão é dada pelo número de vértices da superfície.

A fim de representar superfícies com bordo, um corner $c$ que for oposto à uma aresta de bordo da superfície terá o seu corner oposto definido com -1 , ou seja, $O[c]=-1$.

A Figura 3.2 ilustra a configuração das tabelas $O$ e $V$ para uma malha triangular simples. O exemplo foi adaptado de (Vieira, 2003), onde este usava a malha de um tetraedro como exemplo. Optamos aqui por uma malha aberta para ficar evidente a representação de bordas. 


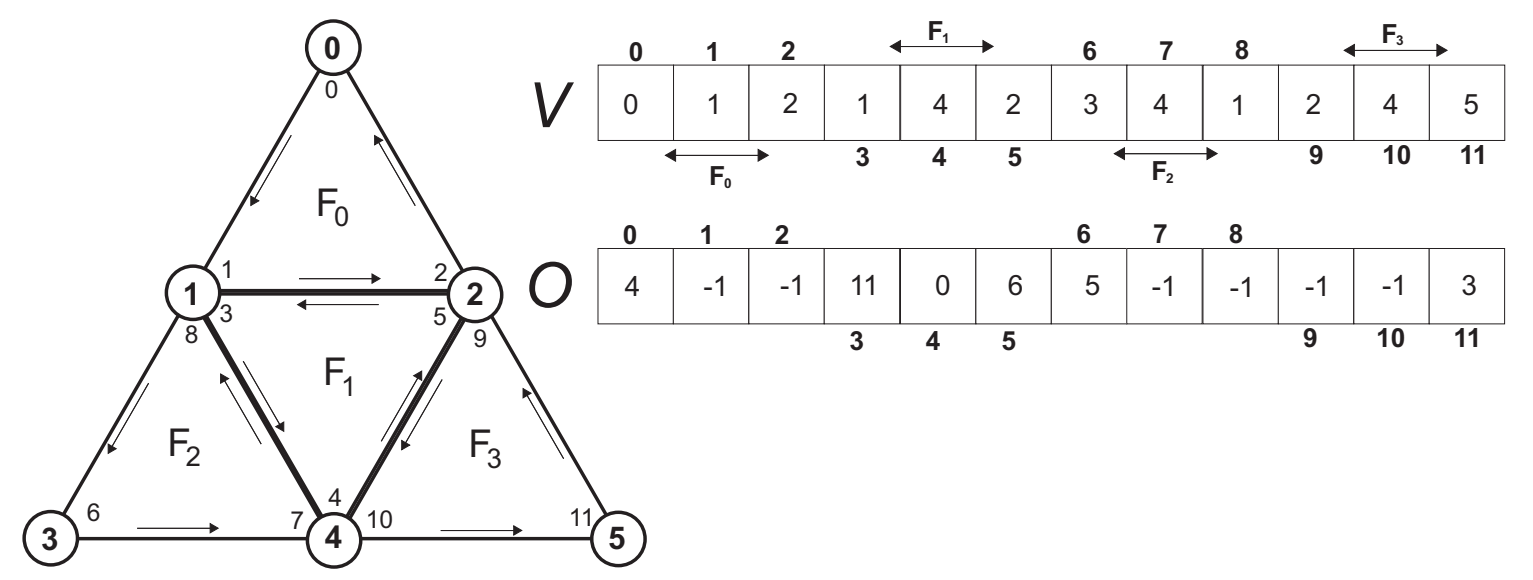

Figura 3.2: Representação de uma malha de triângulos usando Corner Table. Adaptado de (Vieira, 2003)

Como os corners são indexados de forma consecutiva e seguindo a orientação da face, é possível escrever rotinas para obtenção do corners denominadas next e previous de um corner c, usando apenas operações entre inteiros (Vieira, 2003) :

$$
\begin{aligned}
& n(c)=\operatorname{next}(c)=3\lfloor c \div 3\rfloor+(c+1) \bmod 3 \\
& p(c)=\operatorname{prev}(c)=3\lfloor c \div 3\rfloor+(c+2) \bmod 3
\end{aligned}
$$

Com estes operadores, as faces da direita e da esquerda podem ser obtidas da seguinte forma:

$$
\begin{gathered}
l(c)=\operatorname{left}(c)=O[\operatorname{prev}(c)] \\
r(c)=\operatorname{right}(c)=O[\operatorname{next}(c)]
\end{gathered}
$$

A Figura 3.3 ilustra os corners que resultam das operaçãos acima a partir de suas aplicações no corner $c$.

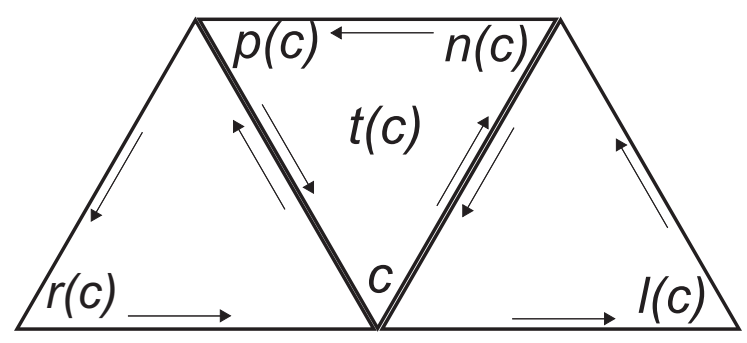

Figura 3.3: Corners $n(c), p(c), l(c)$ e $r(c)$ que resultam das operações de next, previous, left e right, respectivamente. $\mathrm{O}$ índice do triângulo ao qual pertence o corner c, é denotado por $t(c)$. Adaptado de (Rossignac et al, 2001) e (Vieira, 2003)

O leitor ao olhar a Figura 3.3, pode imaginar que os corners $l(c)$ e $r(c)$ estão trocados, uma vez que aparentemente $l(c)$ está à direita e $r(c)$ está á esquerda de $c$. No entando, esta notação pode ser compreendida com base nas regras da mão direita 
e esquerda, ou seja, partindo de $c$ e aplicando a regra da mão direita seguindo a orientação do triângulo se obtém o corner $r(c)$ e da mesma forma aplicando a regra da mão esquerda se obtém o corner $l(c)$.

Note que os corners, representados na Figura 3.2, não são gravados de fato. Um corner é o índice de um vértice armazenado na lista de triângulos, de forma que este existe de forma implícita.

A conectividade da malha triangular pode ser completamente representada pelas tabelas $O$ e $V$. Além disso, a tabela $G$ guarda os atributos de cada vértice, assim como sua geometria. Com as tabelas $O$ e $V$ é possível recuperar a vizinhança de um vértice a partir de um corner $c$, no entanto, a operação de recuperar um corner de um vértice tem custo linear, de forma que aplicações que tem como entrada os vértices da malha podem ter problemas de eficiência. Uma solução para este problema foi prosposta por (Vieira, 2003). Neste caso, a estrutura original (Rossignac et al, 2001) seria modificada para ter mais uma tabela $C$, do tamanho da lista de vértices, que armazenaria para cada vértice um dos seus corners incidentes.

A fim de representar de forma implícita o bordo da superfície, a tabela $C$ pode ser construída de forma que se um vértice pertence ao bordo da superfície, o corner $c$ associado a ele a ser armazenado é aquele em que next $(c)$ leva a um outro vértice de bordo.

A Figura 3.4 ilustra o exemplo da Figura 3.2 com a inclusão da tabela $C$.

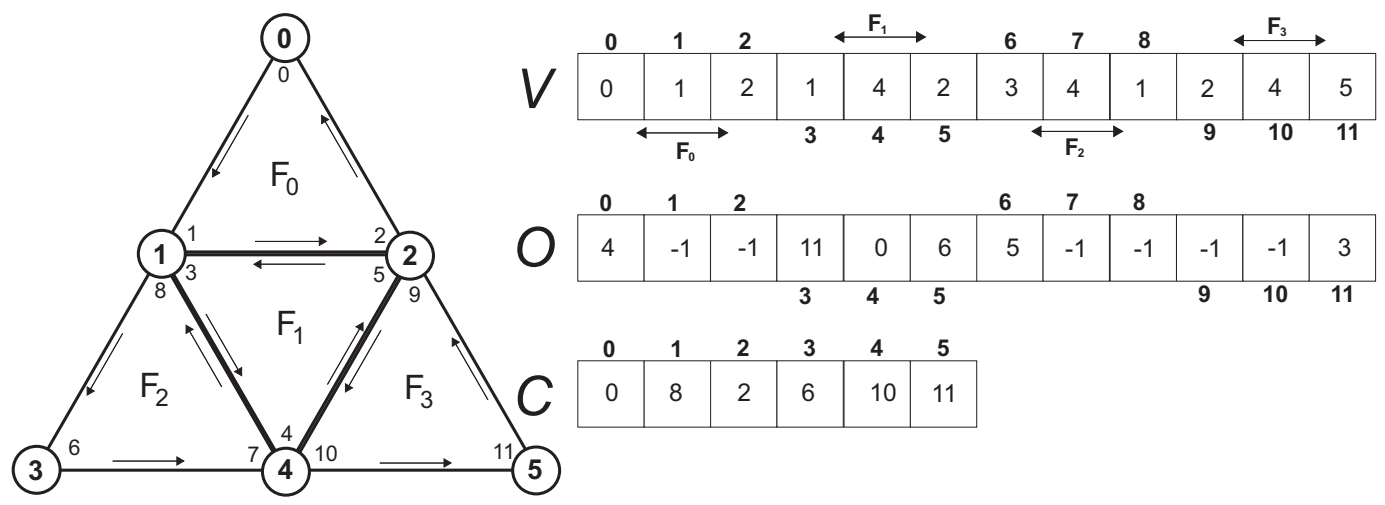

Figura 3.4: Representação incluindo a tabela $C$

\subsection{2}

\section{Incidências e Adjacências}

Nesta seção mostra como obter as relações de incidência e adjacência necessárias para identificação da conectividade dos vértices, arestas e das faces de uma malha triangular usando a Corner Table. Estas buscas topológicas foram implementadas por (Vieira, 2003), e são repoduzidas aqui para facilitar a leitura.

Em (Vieira, 2003) a notação a seguir é utilizada:

V: um grupo de vértices; 
$v$ : um determinado vértice;

E: um grupo de arestas;

$e$ : uma determinada aresta;

F: um grupo de faces

$f$ : uma determinada face;

$\{$ grupo\}: um conjunto não ordenados de elementos;

A Corner Table não tem uma representação explícita para as arestas, uma vez que uma aresta pode ser representada implicitamente por um dos seus corners opostos. Um corner oposto $c$ representa a aresta formada pelos vértices $V[$ next $(c)]$ e $V[\operatorname{prev}(c)]$ (Vieira, 2003). Note que se a aresta $e$ for interna à malha, ela pode ser representada por dois corners e quando esta pertence ao bordo da superfície pode ser representada apenas por um corner.

Na Figura 3.5 a aresta $e_{1}$ pode ser representada pelos corners 9 e 14, já a aresta $e_{2}$ só pode ser representada pelo corner 1 .

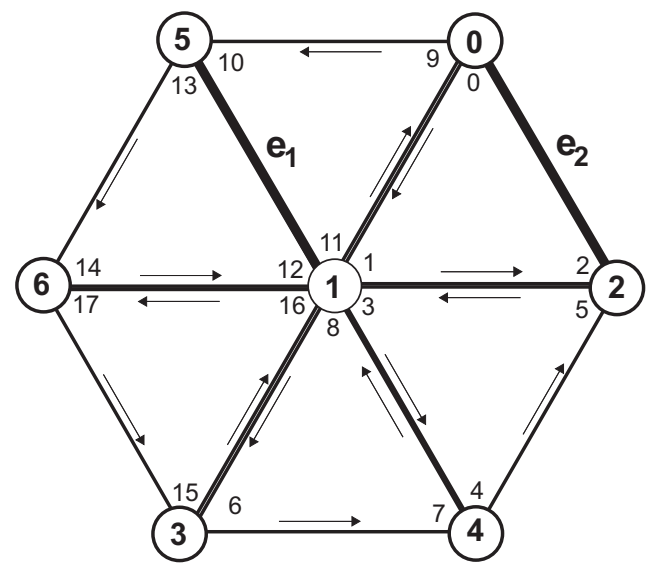

Figura 3.5: A estrela de um vértice e a representação implícita de arestas

Para simplificar os algoritmos vamos introduzir uma função que retorna a aresta oposta a um corner $c$ (ver Algoritmo 1).

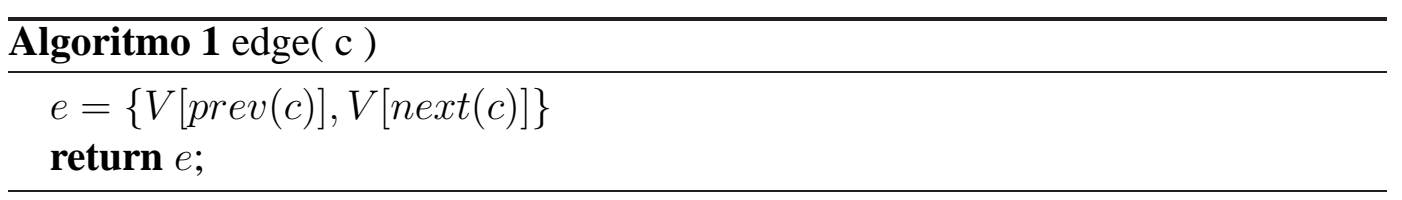

\section{Vizinhanças de um Vértice}

Dado um vértice $v$, pode-se obter um corner $c$ associado a $v$ da tabela $C$, ou no caso desta não existir, percorrer a tabela $V$ em tempo linear. Dado $c$, podese percorrer a estrela de $v$, obtendo os conjuntos de vértices $\{\mathbf{V}\}(v)$ adjacentes a $v$ e $\operatorname{arestas}\{\mathbf{E}\}(v)$ e faces $\{\mathbf{F}\}(v)$ incidentes a $v$ em tempo $O(\operatorname{Degree}(v))$ (Vieira, 2003)( ver Algoritmo 2). 


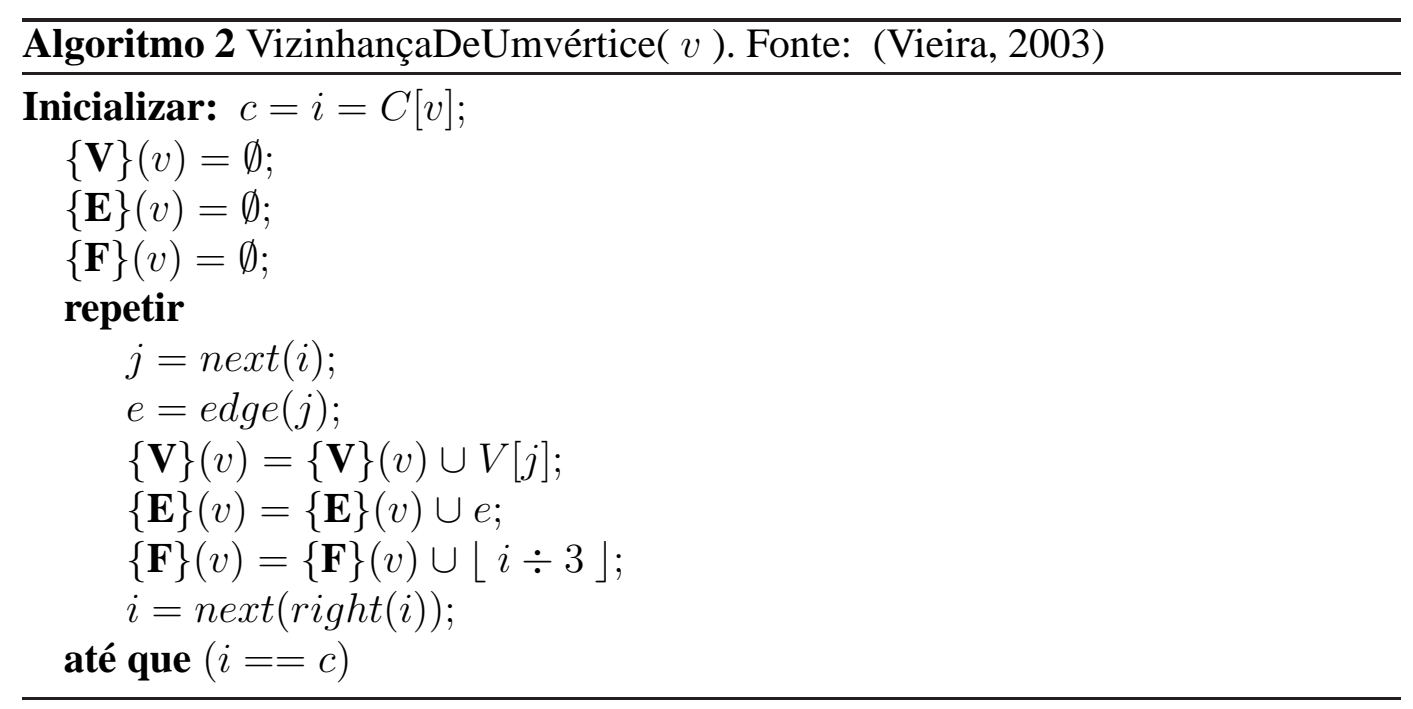

Por simplicidade o Algoritmo 2 não trata superfícies de bordo. O laço que gira na estrela do vértice pode ser interrompido ao se deparar com o bordo da superfície, neste caso o a busca deve ser retomada pelo corner inicial, mas girando no sentido oposto desta vez.

\section{Vizinhanças de uma aresta}

Dada uma aresta $e$, obtemos o corner $c$ oposto a $e$, assim pode-se obter os conjuntos de vértices $\{\mathbf{V}\}(e)$ e faces $\{\mathbf{F}\}(e)$ incidentes a $e$ e $\operatorname{arestas}\{\mathbf{E}\}(e)$ adjacentes a $e$ em tempo constante (Vieira, 2003)( ver Algoritmo 3).

Note que é necessário um tempo linear obter um corner c oposto a uma aresta $e$, uma vez que os corners deveriam ser visitados até que se encontrasse o corner $c$ oposto a aresta que tenha os mesmos vértices extremos que $e$. Assim como a tabela $C$, que associada um corner para cada vértice, uma nova tabela pode ser alocada para associar um corner a cada aresta, no entanto, esse tipo de consulta explícita não será necessária em nossa aplicação, não necessitando assim desta tabela extra.

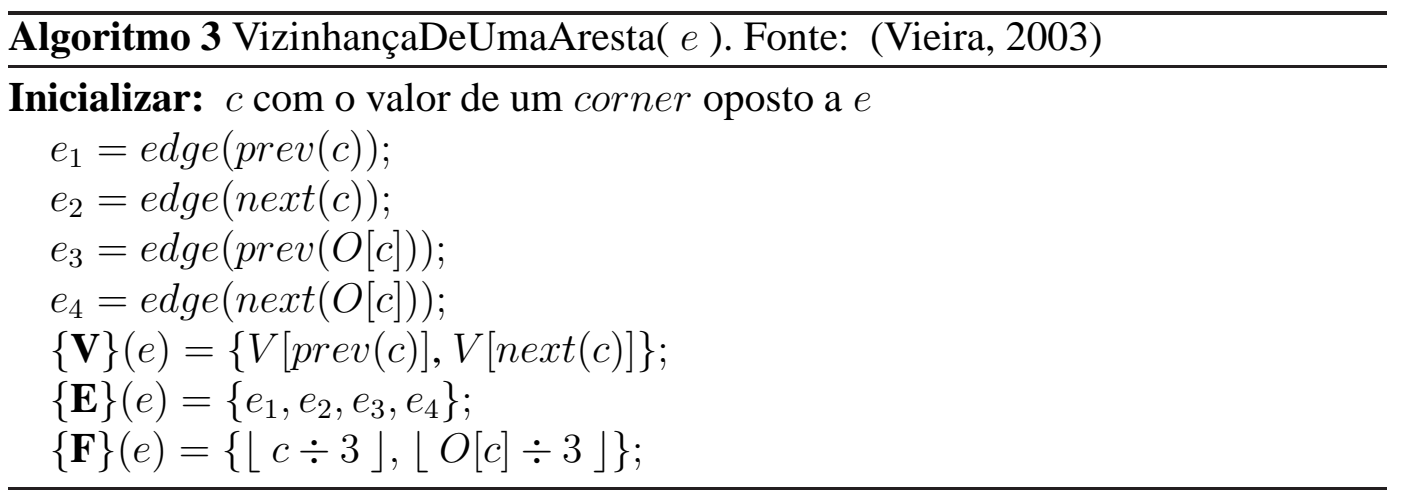




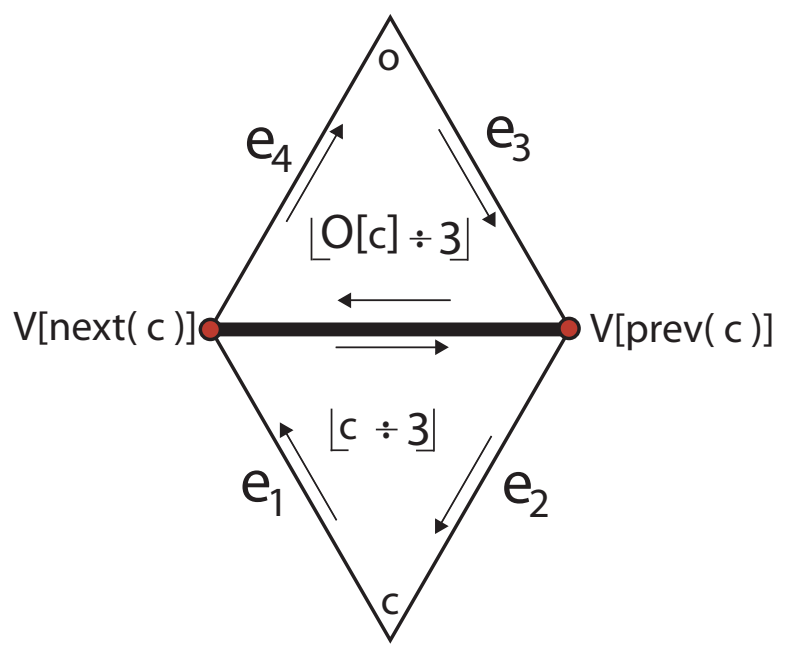

Figura 3.6: Vizinhança de uma aresta

\section{Vizinhanças de uma face}

Dada uma face $f$, obtemos o primeiro corner $c$ associado a ela fazendo $c=3 f$, e então pode-se obter os conjuntos de vértices $\{\mathbf{V}\}(f)$ e arestas $\{\mathbf{E}\}(f)$ incidentes a $f$ e faces $\{\mathbf{F}\}$ adjacentes a $f$ em tempo constante (Vieira, 2003)( ver Algoritmo 4).

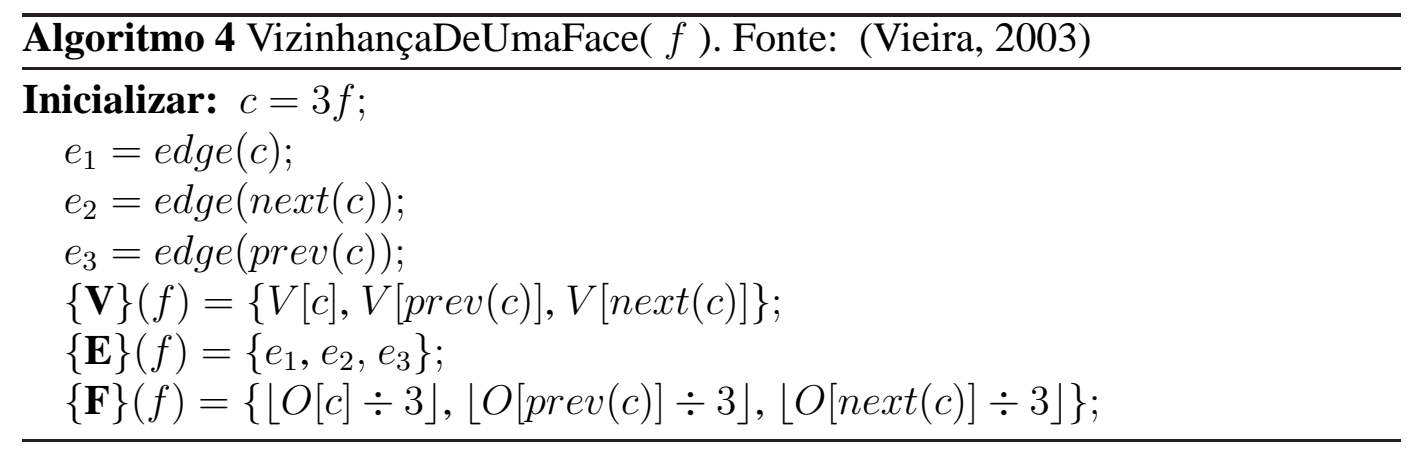

\section{2}

\section{CHE - Compact Half-Edge}

A CHE, ou Compact Half-Edge, é uma estrutura de dados topológica, proposta por (Ferreira, 2006), que tem como uma de suas principais características o fato de ser escalonável, além de usar o conceito de half-edge. A estrutura é dividida em quatro níveis, de forma que, à medida que haja disponibilidade física, uma quantidade adicional de mémoria pode ser alocada para obter melhor performace nas consultas topológicas, ou por outro lado, liberar mémoria com 
o objetivo de processar modelos maiores, com o custo de perder desempenho no tempo de resposta das informações topológicas. Assim, a estrutura busca um balanceamento entre o uso de memória e a eficiência da representação topológica.

A seguir, serão descritos os quatro níveis da $\mathrm{CHE}$, assim como as informações que são acrescidas a cada nível para melhorar a efiências nas consultas topológicas. A descrição a seguir foi retirada de (Ferreira, 2006), onde pode ser encontrada uma descrição mais deltalhada da CHE.

\subsection{1}

\section{Nível 0: Sopa de Triângulos}

No nível 0 são armazenadas apenas as informações essenciais para representação da malha triangular, como a geometria dos vértices e a lista de triângulos em si. Neste nível não há intenção de representar de forma eficiente as relações de incidência e adjacência, uma vez que não são guardadas informações de vizinhança entre os triângulos de forma explícita.

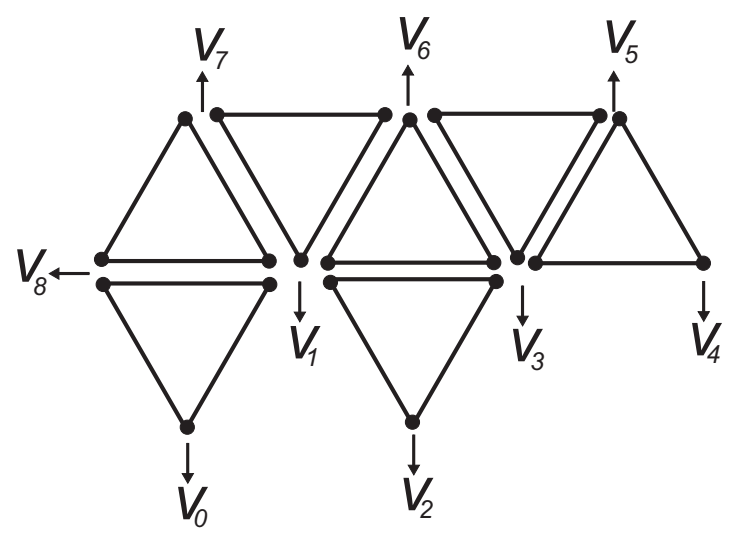

Figura 3.7: Nível 0 da CHE: Sopa de triângulos. Adaptado de (Ferreira, 2006)

A geometria dos vértices é armazenada em uma tabela $G$, que tem uma entrada para cada vértice. A lista contendo os triângulos é armazenada na tabela $V$. Nesta tabela é amazenado para cada half-edge o índice do seu vértice inicial, ou seja, o inteiro $v=V[h e]$ corresponde ao índice do vértice inicial de uma half-edge he.

De forma semelhante à Corner Table, cada triângulo é representado por 3 half-edges consecutivas que são indexadas de acordo com a sua orientação. Assim, as half-edges 0,1 e 2 pertencem ao triângulo de índice 0 , as half-edges 3 , 4 e 5 pertencem ao triângulo de índice 1, e assim por diante. Uma half-edge de índice he pertence ao triângulo $\lfloor h e \div 3\rfloor$ e um triângulo de índice $t$ contém as half-edges de índices $3 t, 3 t+1$ e $3 t+2$. Além disso, pode-se obter as half-edges denominadas next e previous de uma half-edge he usando apenas operações de inteiros, assim como na Corner Table: 


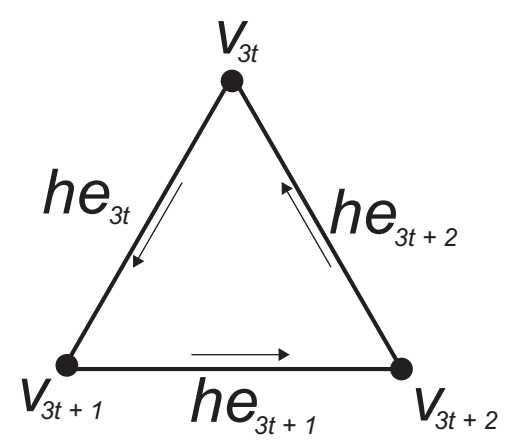

Figura 3.8: Relação entre vértice e half-edge. Adaptado de (Ferreira, 2006)

$$
\begin{aligned}
& h_{n}=\operatorname{next}(h e)=3\lfloor h e \div 3\rfloor+(h e+1) \bmod 3 \\
& h_{p}=\operatorname{prev}(h e)=3\lfloor h e \div 3\rfloor+(h e+2) \bmod 3
\end{aligned}
$$

\subsection{2}

\section{Nível 1: Adjacência entre Triângulos}

No nível 1, a CHE passa a tratar a malha como um conjunto de triângulos conectados (Figura 3.9). Isso é feito pela adição de um novo vetor $O$, que tem mesma dimensão da lista triângulos, e que em cada posição indica o índice da halfedge oposta. Uma half-edge $h$ é oposta a half-edge he se $h$ tem os mesmos vértices de he, mas orientação oposta (Figura 3.10). Quando he for incidente à uma aresta de bordo, esta não terá uma half-edge oposta. Neste caso, $O[$ he $]=-1$.

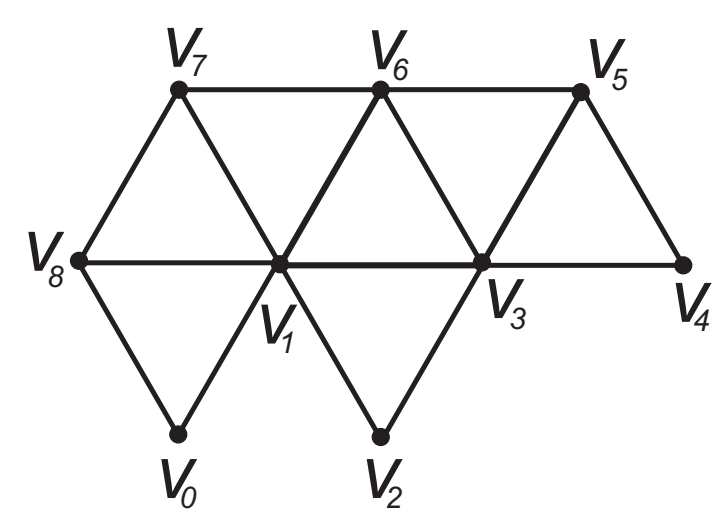

Figura 3.9: Nível 1 da CHE: Adjacência entre os triângulos. Adaptado de (Ferreira, 2006)

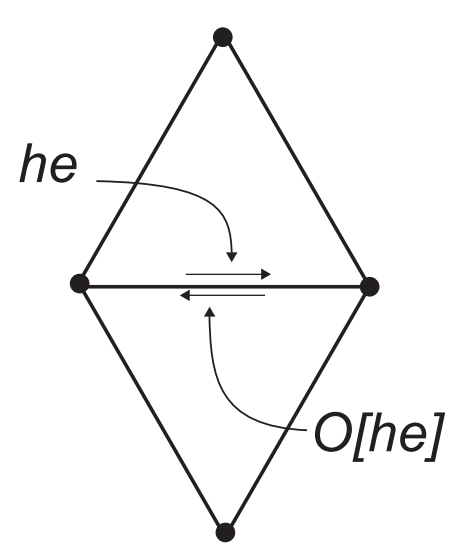

Figura 3.10: Half-edges opostas. Adaptado de (Ferreira, 2006)

\section{2 .3}




\section{Nível 2: Representação das Células}

No nível 2 da CHE, é acrescentada uma representação explícita para cada célula da malha. Tal representação é feita por meio de duas novas tabelas, que serão chamadas de $E H$ e $V H$.

A tabela $E H$ tem a sua dimensão determinada pelo número de arestas, e associa cada aresta com uma de suas half-edges incidentes, já que a outra pode ser obtida através do vetor $O$. Essa representação explícita permite que atributos relacionados às arestas possam ser armazenados em uma outra tabela, que pode ter a mesma indexação da tabela $E H$.

Note que os vértices que compõe a aresta são facilmente recuperados através dos vértices iniciais de suas half-edges.

Já na tabela $V H$, cada vértice é associado à uma de suas half-edges incidentes. Como podem existir várias half-edges que podem ser associadas ao vértice, (Ferreira, 2006) definiu um critério de escolha. Se o vértice é de bordo, então a half-edge escolhida será a half-edge de bordo que tem o vértice como vértice inicial. Se o vértice for de interior, a half-edge armazenada poderá ser qualquer half-edge incidente que tenha o vértice como vértice inicial.

Desta forma, se torna bastante simples classificar os vértices da malha como interior ou de bordo. Dado um vértice $v$, basta verificar se $O[V H[v]]=-1$. Em caso afirmativo, o vértice é de bordo.

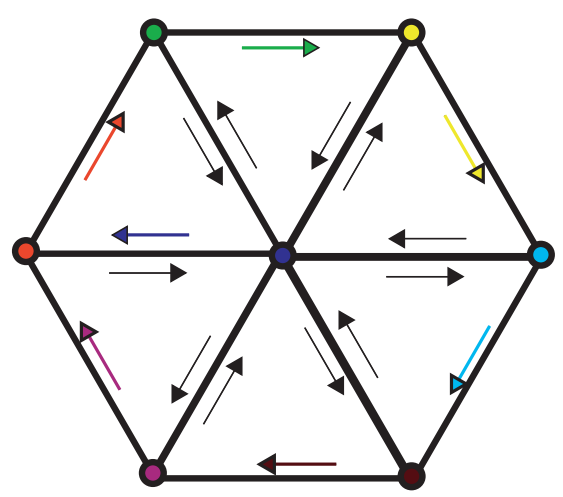

Figura 3.11: Escolha das half-edges para serem armazenadas na tabela $V H$. Adaptado de (Ferreira, 2006)

\subsection{4}

\section{Nível 3: Representação das Curvas de Bordo}

No nível 3 da CHE, é adicionada uma estrutura para representação explícita para curvas de bordo de superfícies. Para isto, uma nova tabela é $C H$ é criada. Cada posição desta tabela armazena uma curva de bordo. Cada curva de bordo é representada por uma das half-edges, uma vez que a partir desta, todas as outras 
podem ser obtidas de forma eficiente através das regras aritméticas e da tabela $O$, como no Algoritmo 5.

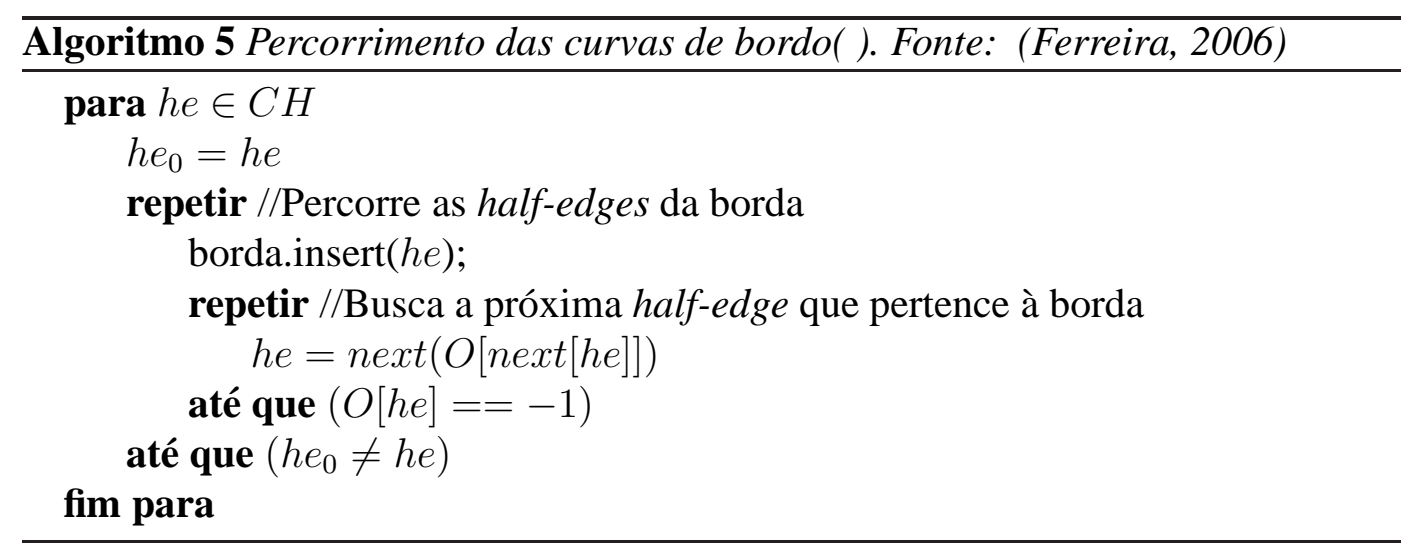

A localização das arestas na curva de bordo pode ser otimizada através da de um atributo gravado na tabela $O$. Nesta tabela pode ser armazenado o índice da componente de bordo a qual pertence a half-edge de bordo. Por exemplo, se uma half-edge de bordo he pertence à componente de número 3 , então $O[h e]=-3$.

\subsection{5}

\section{Exemplo}

A seguir é mostrado um exemplo de uma simples malha triangular representada pela CHE. Neste exemplo é mostrada uma CHE nível 3, com todas as tabelas preenchidas como definido acima.
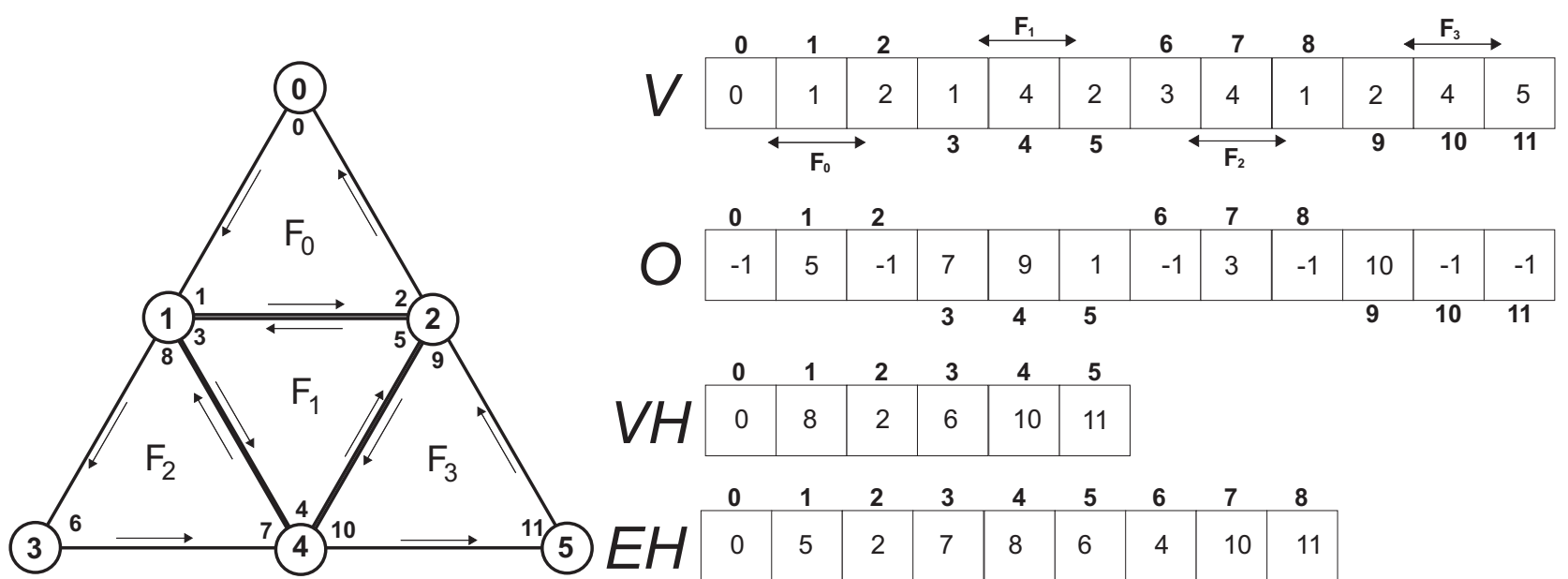

\begin{tabular}{l|l|l|l|l|l|l|}
\multicolumn{1}{c}{0} & 1 & 2 & 3 & 4 & 5 \\
\hline & 0 & 8 & 2 & 6 & 10 & 11 \\
\hline
\end{tabular}

\begin{tabular}{|l|l|l|l|l|l|l|l|l|l|}
\hline & 0 & 1 & 2 & 3 & 4 & 5 & 6 & 7 & 8 \\
\hline & 0 & 5 & 2 & 7 & 8 & 6 & 4 & 10 & 11 \\
\hline
\end{tabular}

$\mathrm{CH} \stackrel{0}{6}$

Figura 3.12: Exemplo de uma malha representada com a CHE 


\section{3}

\section{Corner Table vs CHE}

A CHE pode ser vista como uma evolução da Corner Table, com um poder de representação maior e com maior eficiência em algumas operações topológicas, como recuperar uma curva de bordo, e ainda tem a propriedade de ser escalonável. Este trabalho poderia ser realizado usando ambas as estruturas, no entanto optamos por usar a Corner Table por ser mais simples, permitir implementações eficientes das buscas topológicas que necessitamos e satisfazer a principal condição do nosso problema, que é o uso de pouca mémoria. 


\section{4 Operadores na Corner Table}

Neste capítulo são apresentadas as implementações usando a Corner Table dos operadores utilizados para realizar as subdivisões global e adaptativa e o mecanismo de undo/redo. São eles: edge split e seu inverso edge weld, edge flip e uma implementação de um edge flip inverso. O motivo da necessidade da implementação de um flip inverso está detalhado na seção 4.3 .

Estes operadores podem ser classificados como operadores estelares, que por sua vez tem propriedades importantes, apresentadas superficialmente na seção 4.1.

\section{1}

\section{Operadores Estelares para Malhas Triangulares}

De acordo com (Velho, 2003), teoria estelar estuda as equivalências entre complexos simpliciais, tendo como principal resultado o seguinte teorema:

Teorema 4.1 Duas n-variedades combinatórias são linearmente homeomorfas por partes, se e somente se, são relacionadas por uma sequência de operações estelares elementares (Newman, 1926) (Pachner, 1991).

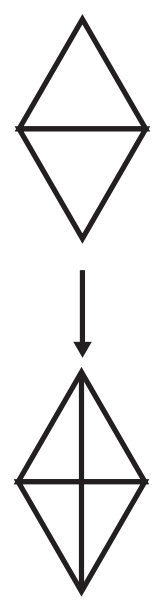

(a)Edge Split / Edge Weld

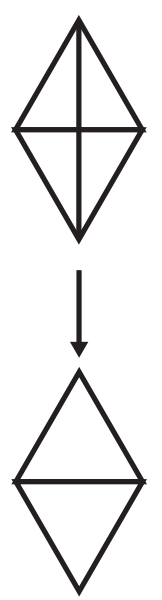

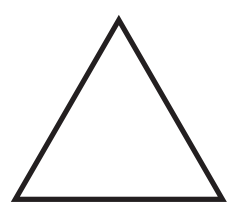
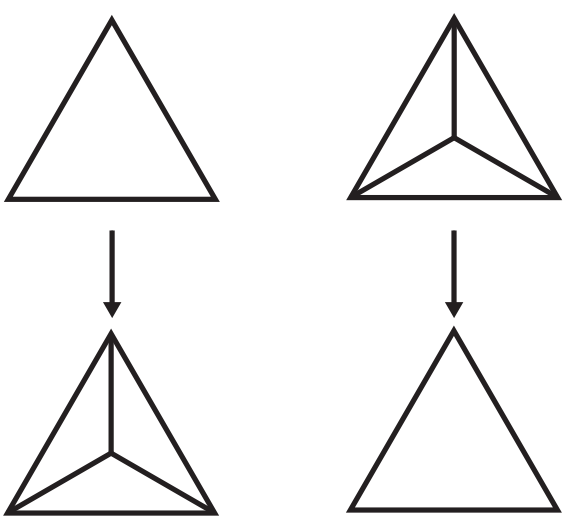

(b)Face Split / Face Weld

Figura 4.1: Operações estelares de subdivisão. Fonte: (Velho, 2003)

Em outras palavras, o teorema 4.1 diz que os operadores estelares realizam transformações entre manifolds combinatórios equivalentes. Este resultado é muito 
importante, uma vez que a subdivisão tem o objetivo apenas de refinar a superfície e não de alterar a sua topologia. Usando estes operadores, garantimos que a superfície subdividida é sempre homeomorfa à superfície grosseira inicial.

Para o caso de malhas de triângulos os operadores são os seguintes: face split e seu inverso face weld; edge split e seu inverso edge weld (Velho, 2003)(ver Figura 4.1).

O operador de edge flip também é um operador estelar, uma vez que ele pode ser obtido através de uma composição de edge split e edge weld, como mostrado na Figura 4.2.

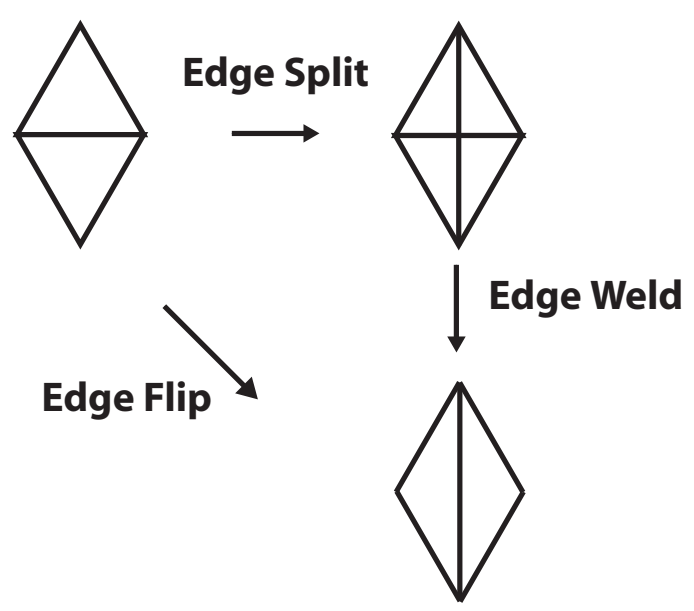

Figura 4.2: Edge Flip como uma composição de edge split + edge weld

Uma descrição mais detalhada sobre teoria estelar pode ser encontrada em (Lickorish1999) e (Velho, 2003).

\section{2 \\ Edge Flip}

Uma implementação para o operador de Edge Flip na Corner Table foi primeiro proposta por (Vieira, 2003). No nosso trabalho utilizaremos uma adaptação desta implementação. Na prática o resultado das duas implementações são equivalentes, há apenas uma translação dos corners dentro das duas faces envolvidas.

O operador de Edge Flip consiste em transformar duas faces adjacentes em outras duas faces também adjacentes pelo swap da aresta em comum às duas.

Seja $e=\langle u v\rangle$ e $s$ e $t$ dois vértices opostos à aresta $e$, como na Figura 4.3. A operação de Edge Flip substitui a aresta $e$ por $\langle s t\rangle$ e as faces incidentes a $e$ por $\langle t s u\rangle$ e $\langle v s t\rangle$. Note ainda que a valência dos vértices $u$ e $v$ diminui, enquanto que a valência dos vértices $s$ e $t$ aumenta.

O Algoritmo 6 realiza a operação de Edge Flip em uma aresta oposta ao corner $c_{0}$. 


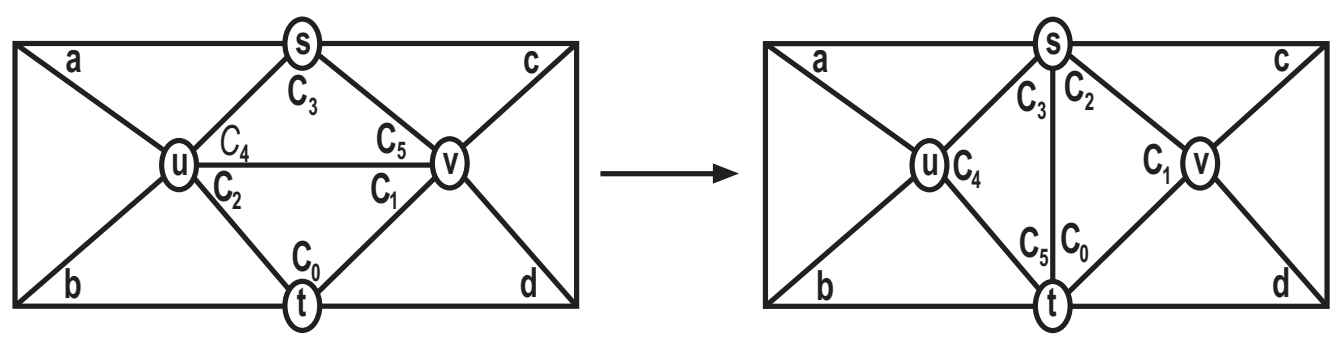

Figura 4.3: Edge Flip na Corner Table

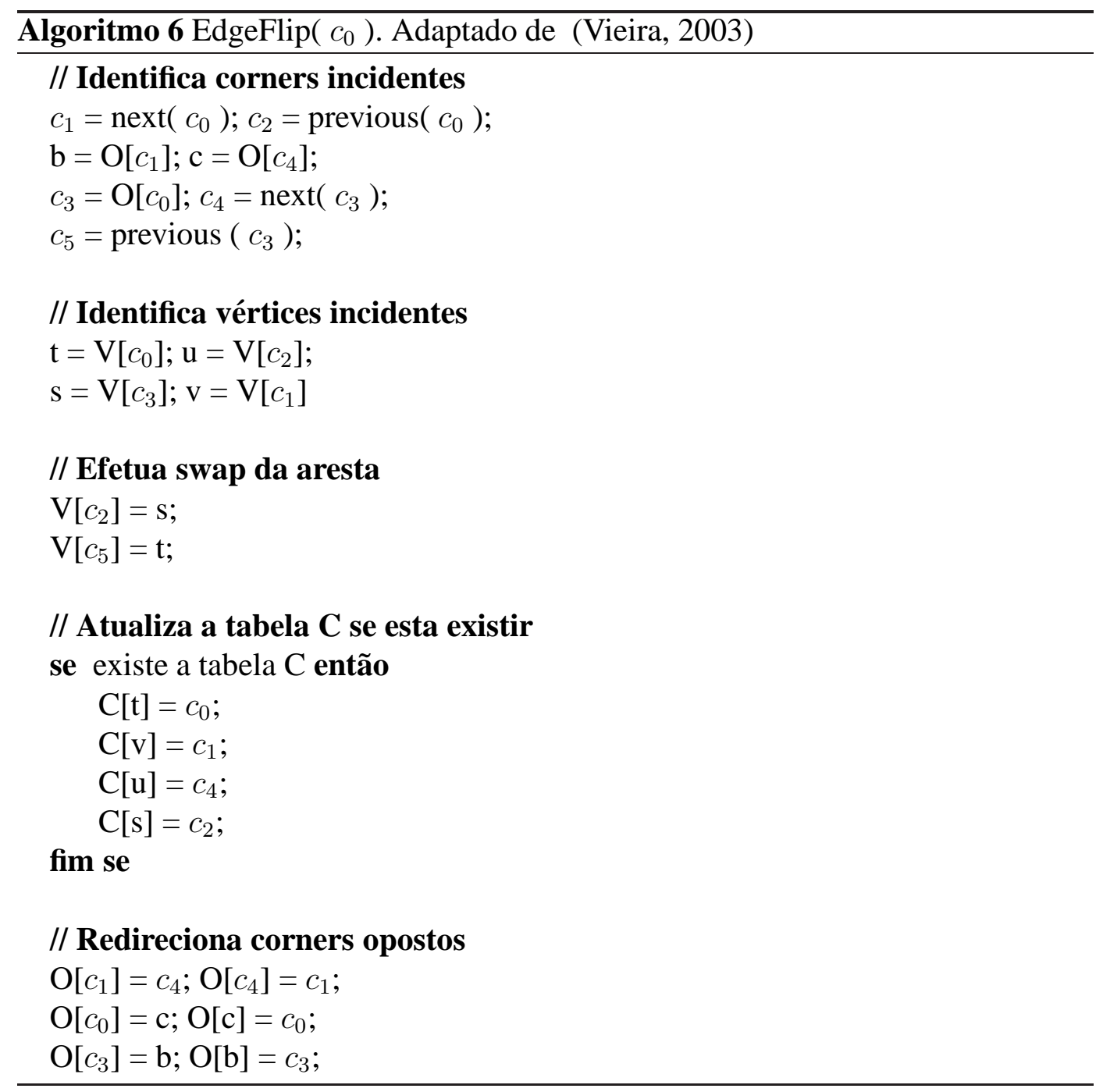

Uma propriedade importante deste operador é que apenas dois corners, que são $\operatorname{prev}\left(c_{0}\right)$ e $\operatorname{prev}\left(O\left[c_{0}\right]\right)$, mudam de vértices, ou seja, os corners $c_{0}$, $O\left[c_{0}\right]$, next $\left(c_{0}\right)$ e $n \operatorname{ext}\left(O\left[c_{0}\right]\right)$ permanecem associados aos mesmos vértices. Esta propriedade serve de base para implementação do algoritmo de subdivisão mostrado no capítulo 5. 


\section{3}

\section{Edge Flip Inverso}

Para implementação de um mecanismo de undo/redo é importante ter operadores inversíveis, mas apesar do operador edge flip ter ele mesmo como seu inverso, a sua aplicação em uma aresta na Corner Table tem o efeito de girar as faces, de forma que a aplicação deste operador em uma aresta duas vezes seguidas produz uma configuração na estrutura de dados diferente da inicial, como mostrado na Figura 4.4.


Figura 4.4: A aplicação do Edge Flip faz com que as faces girem no sentido antihorário

Para voltar ao estado inicial seria necessário a aplicação do operador Edge Flip três vezes em sequência. Uma outra opção seria realizar um swap entre as duas faces e em seguida aplicar o operador Edge Flip, no entanto ainda teriamos duas operações. Por questões de eficiência optamos pela implementação de um operador Edge Flip que faça as faces girarem no sentido horário, como na Figura 4.5.
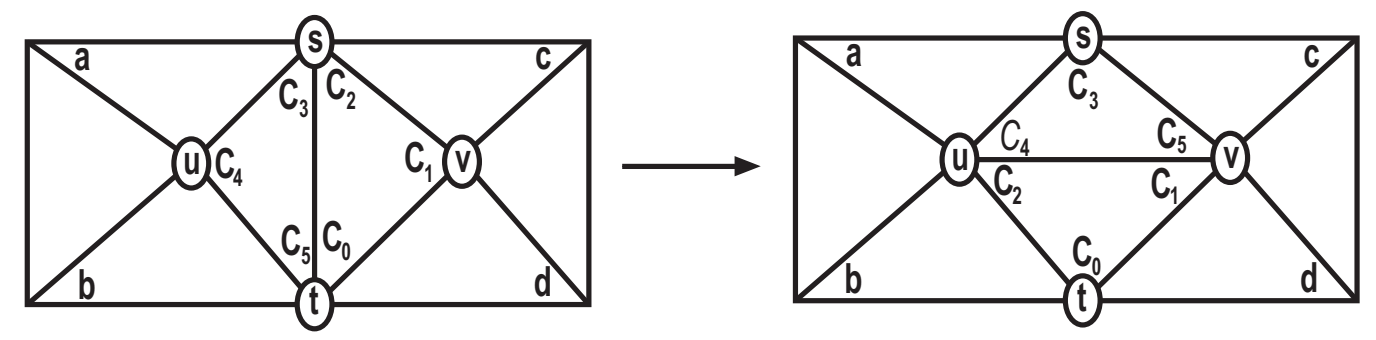

Figura 4.5: Edge Flip inverso

O Algoritmo 7, com pequenas mudanças para o Algoritmo 6, implenta o operador de Edge Flip Inverso. 
Além de retornar à configuração inicial da Corner Table, este operador também mantém os corners $c_{1}, O\left[c_{1}\right]$, prev $\left(c_{1}\right)$, prev $\left(O\left[c_{1}\right]\right)$ associados aos mesmos vértices.

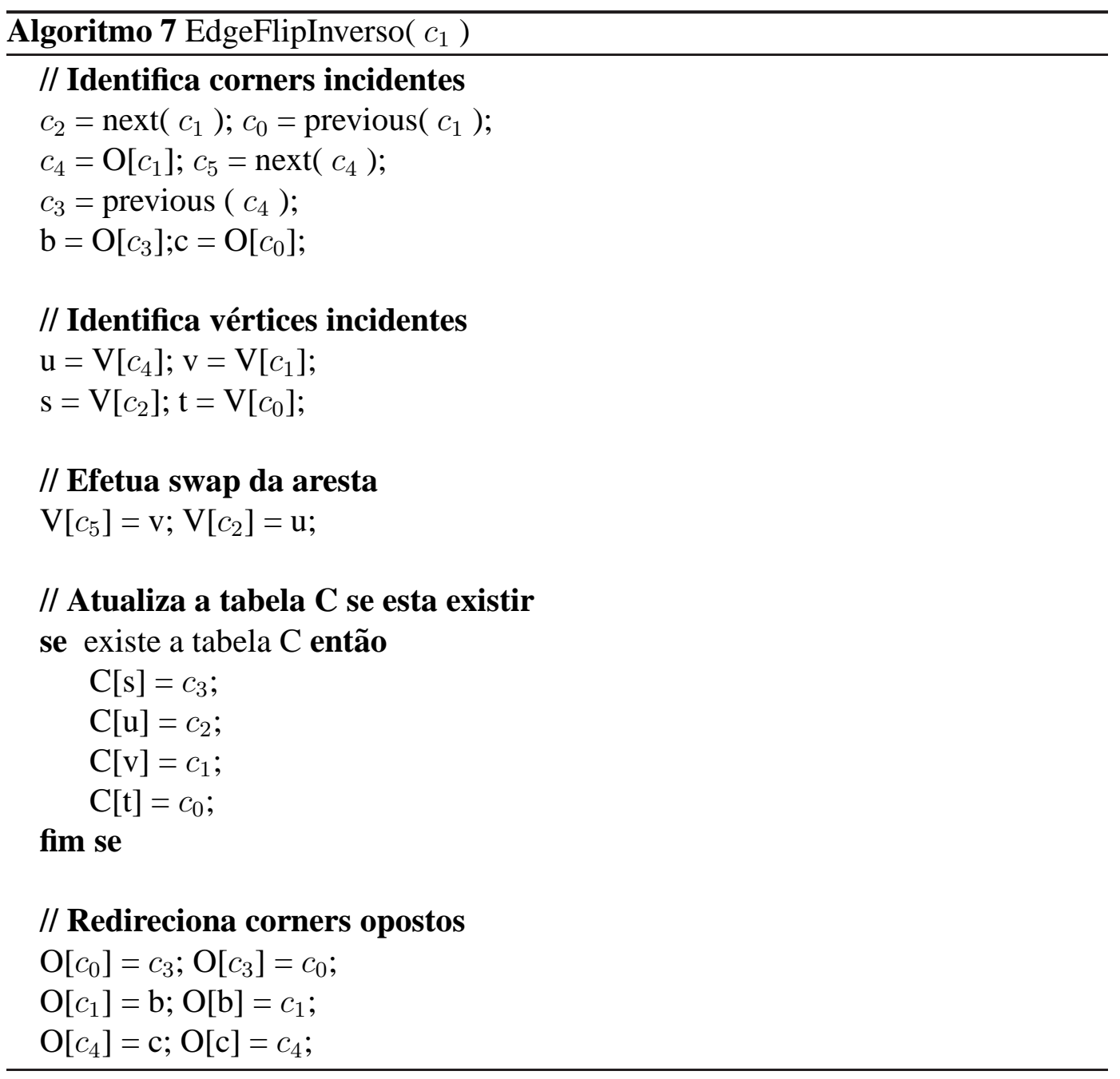

\section{4}

\section{Edge Split}

A operação de Edge Split consiste em transformar duas faces adjacentes em quatro faces pela inserção de um novo vértice sobre a aresta comum às duas.

Seja $e=\langle u v\rangle$ e $s$ e $t$, dois vértices opostos à aresta $e$, como na Figura 4.6. A operação de Edge Split criará um novo vértice $n$ sobre a aresta $e$, e este será conectado aos vértices $s$ e $t$ gerando as novas arestas $e_{1}=\langle n t\rangle$ e $e_{2}=\langle n s\rangle$. Além disso as faces $\langle t v u\rangle$ e $\langle s u v\rangle$ são substituidas pelas faces $\langle t n u\rangle,\langle s n v\rangle,\langle t v n\rangle$ e $\langle s n u\rangle$.

O novo vértice $n$ sempre terá valência 4 , e os vértices $s$ e $t$ terão suas valências aumentadas.

$\mathrm{Na}$ Corner Table, cada face pode ser representada implicitamente por um índice inteiro $f_{i}$. Desta forma, ao subdividir a aresta $e$ existem várias formas de reposicionar as faces $F_{1}$ e $F_{2}$. Para facilitar a implementação dos algoritmos de 

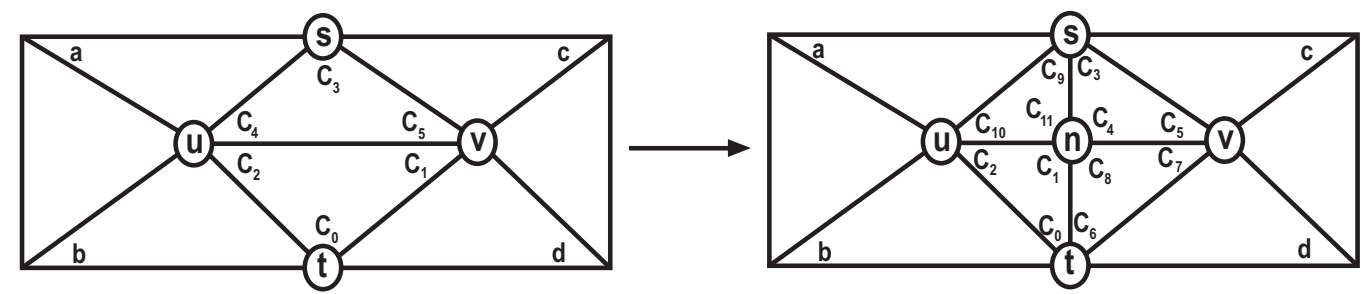

Figura 4.6: Edge Split na Corner Table

subdivisão e do mecanismo de undo/redo, será adotada a convenção exibida na Figura 4.7.

Nesta convenção as faces $F_{1}$ e $F_{2}$ existentes antes de realizar a operação de Edge Split são posicionadas na nova configuração da mesma forma, independente se a operação foi realizada no corner $c$ ou no corner $o$, oposto ao corner $c$.
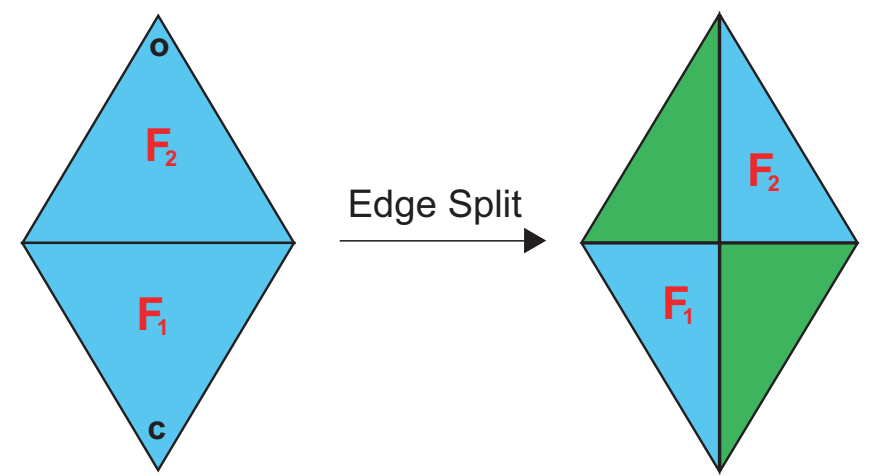

Figura 4.7: Convenção para posicionamento das faces após a realização da operação de Edge Split

O Algoritmo 8 realiza a operação de Edge Split em uma aresta oposta ao corner $c_{0}$, seguindo a convenção adotada.

Ao aplicar o operador de Edge Split, as faces $F_{1}$ e $F_{2}$ ocupam as mesmas posições na tabela $V$, sendo que apenas um dos vértices de cada uma muda durante a operação. Já as duas novas faces são adicionadas no final da tabela $V$. Se não houver espaço a tabela $V$ é realocada de acordo com uma constante $m>1$ definida pelo usuário. Desta forma o novo tamanho da $n$ da tabela a cada vez que esta for realocada é dado por

$$
n=n_{c} * m
$$

onde $n_{c}$ é o número máximo de elementos que cabem na tabela corrente. Se o usuário não definir explicitamente um valor para $m$, este valor é definido como sendo 2. 


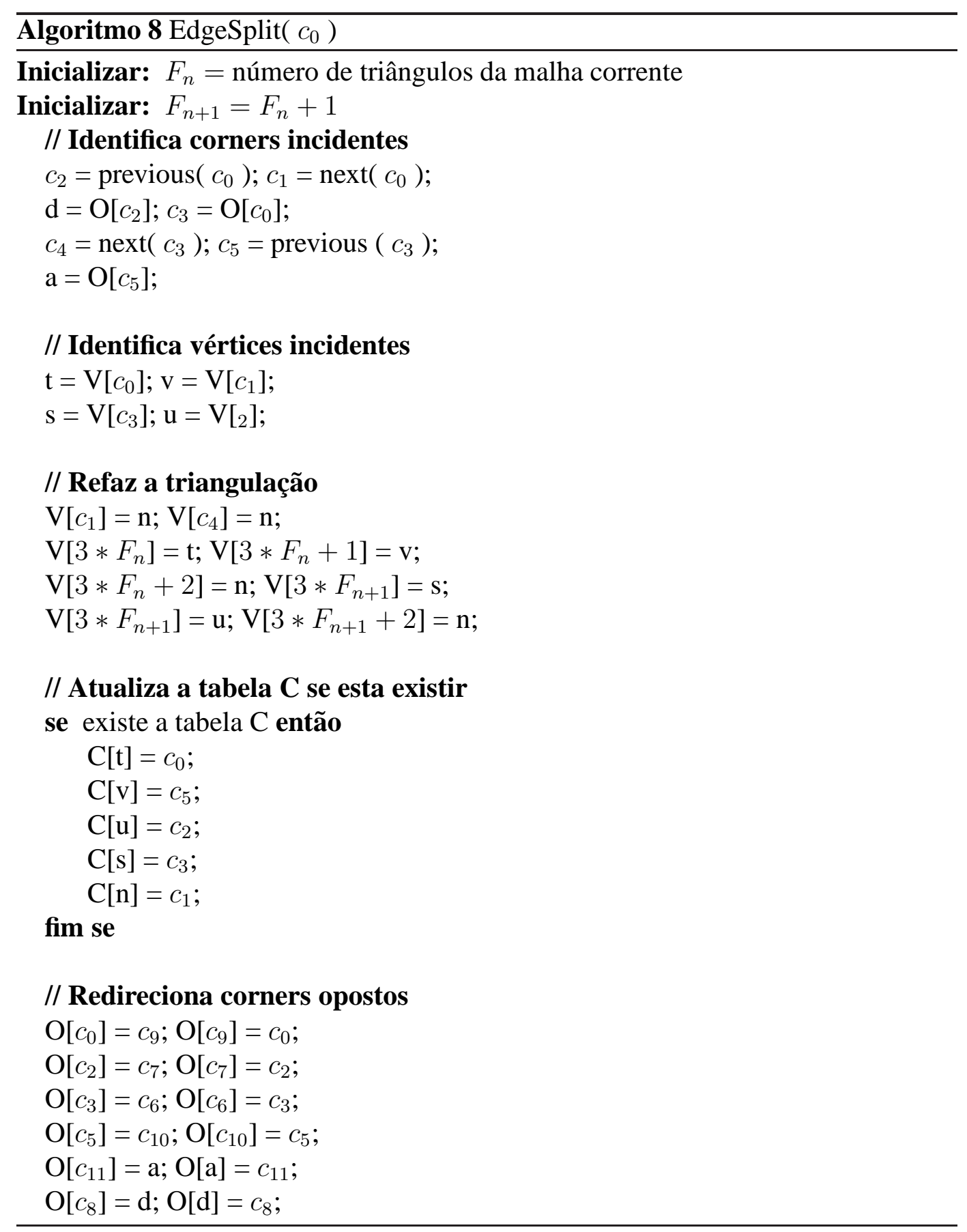

\section{5}

\section{Edge Weld}

Como colocado na seção 4.1, o operador de Edge Weld é inverso ao operador de Edge Split. Desta forma, utilizaremos este operador no mecanismo de undo/redo para reverter o efeito do operador Edge Split.

Uma implementação para este operador foi primeiro proposta por (Vieira, 2003). Assim como no caso do operador de Edge Flip, utilizaremos uma adaptação desta implementação, a fim de garantir que a aplição de um Edge Split 
seguido de um Edge Weld faça com que a estrutura de dados tenha uma configuração igual à aquela de antes da aplicação do operador de Edge Split.
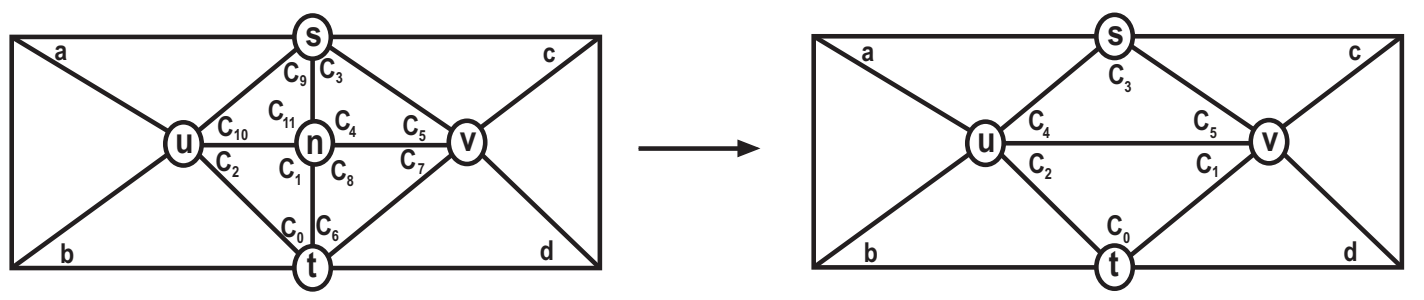

Figura 4.8: Edge Weld na Corner Table

Seja $n$ um vértice de valência 4 , adjacente aos vértices $s, t, u$ e $v$, como na Figura 4.8. O operador Edge Weld remove o vértice $v$ e refaz a triangulação do quadrilátero resultante dividindo-o em duas faces. Com a aplicação o operador a partir do corner $c_{1}$, a arestas $\langle t n\rangle$ e $\langle s n\rangle$ são removidas, de forma que as duas faces resultantes são $\langle t v u\rangle$ e $\langle s u v\rangle$.

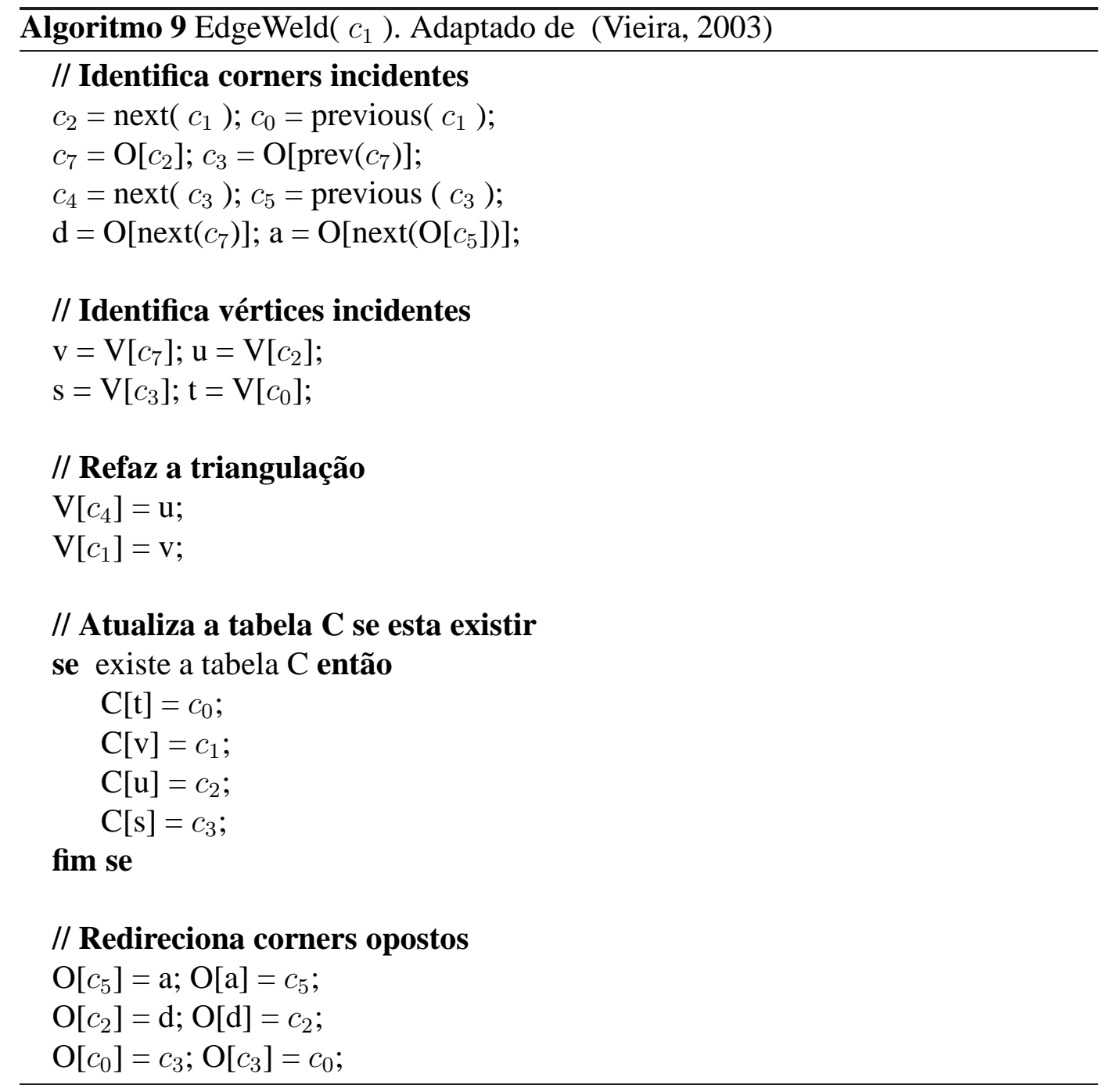

Como este operador é utilizado para reverter o operador de Edge Split, é garantido que as duas faces removidas estão no fim do vetor $V$, como visto na seção 
4.4. Desta forma, para remover as duas faces, basta decrementar a variável que guarda o número de faces. Isto evita a necessidade de uma operação de garbagecollector para redimensionar as tabelas retirando os espaços não utilizados, uma vez que as tabelas são sempre contínuas. Para evitar que um grande montade de memória esteja alocada para o vetor $V$ sem que este esteja utilizando, o vetor $V$ é realocado sempre que

$$
\frac{n_{f}}{n_{c}}<\frac{1}{m}
$$

onde $n_{f}$ é o número de faces da malha corrente, $n_{c}$ é o número de máximo de faces que cabem na estrutura sem a necessidade dos vetores serem realocados e $m$ é como definido na seção 4.4. O Algoritmo 9 implementa o operador de Edge Weld na Corner Table. 


\section{5 \\ Subdivisão em Corner Table}

Neste capítulo será apresentado uma forma de realizar o refinamento 1 para 4 pela composição de operadores estelares (seção 5.1), além de algoritmos para realização de subdivisão global (seção 5.2) e subdivisão adaptativa (seção 5.3) e uma forma de realizar undo/redo (seção 5.4) para estas subdivisões usando Corner Table. Os esquemas de subdivisão implementados usam a operação de refinamento 1 para 4 que foi apresentado no capítulo 2, mas a forma como a geometria dos novos vértices e calculada é independente. Desta forma, o usuário deve fornecer a geometria dos novos vértices. Isto proporciona uma flexibilidade maior, uma vez que a geometria pode ser definida de acordo com as necessidades do modelo, ou através de métodos como Loop e Butterfly.

\section{1}

\section{Composição de operações}

Diferente de estruturas de dados topológicas como Half Edge e Winged Edge, a Corner Table não representa malhas híbridas, uma vez que esta representa apenas elementos triangulares, sem vértices $T$. Desta forma, qualquer passo intermediário na Corner Table não pode criar vértices $T$ e deve produzir apenas elementos triangulares, a fim de manter uma estrutura consistente durante toda execução do algoritmo. Para garantir tal restrição, a subdivisão 1 para 4 (Figura 5.1) é realizada pela composição de operações estelares elementares.

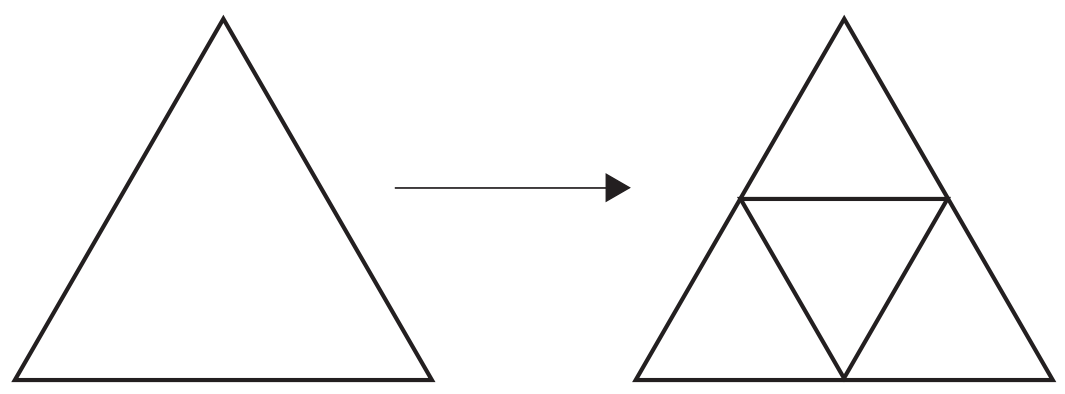

Figura 5.1: Subdivisão 1 para 4

Esta operação pode ser dividida em 4 operações básicas. Uma sequência com três operações de Edge Split e ao final, uma operação de Edge Flip. As operações de Edge Split subdividem as três arestas do triângulo, e a operação de Edge Flip é 
realizada sobre a aresta criada em decorrência do primeiro Edge Split realizado na face. É importante frisar que o operador Edge Flip deve ser aplicada somente ao final. Esta sequência de passos é ilustrada, para a subdivisão de um único triângulo, na Figura 5.2.
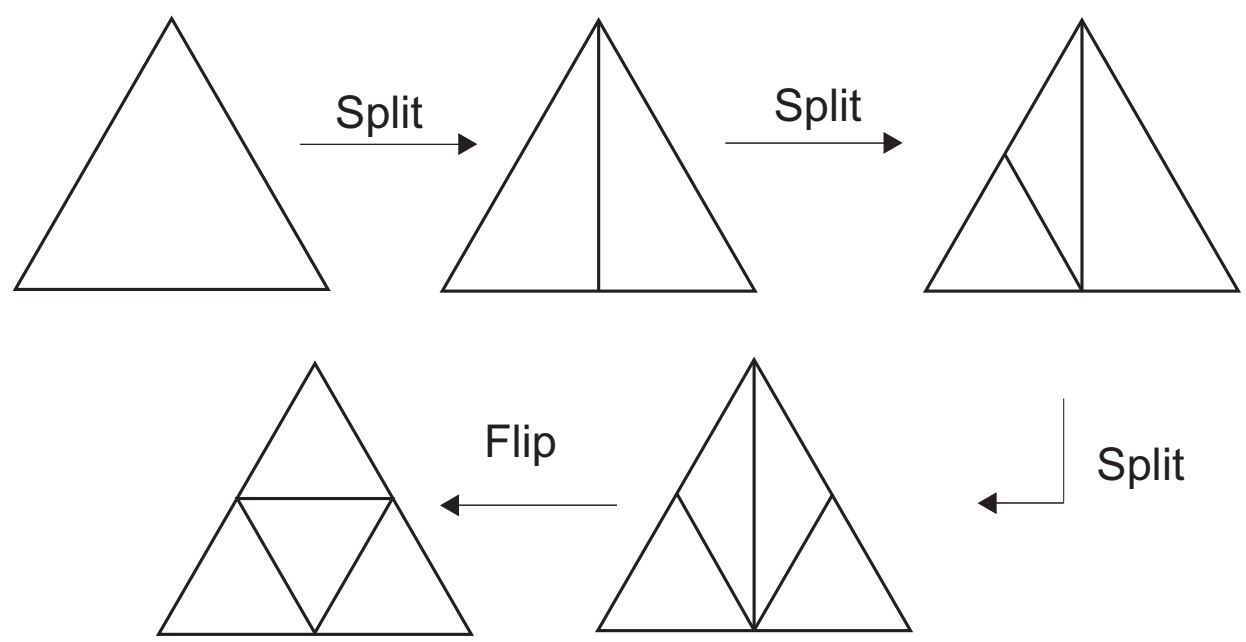

Figura 5.2: Subdivisão 1 para 4 como uma sequência de 3Edge Split + Edge Flip

A fim de manter uma malha sem vértices T, uma operação de Edge Split deve ser realizada também na face adjacente à aresta subdividida, isso evita a criação de vértices em $T$, mas obriga a subdivisão das faces opostas, como ilustrado na Figura 5.3. Note que ao subdividir as faces adjacentes, o que acontece de fato é uma triangulação simples (capítulo 2).
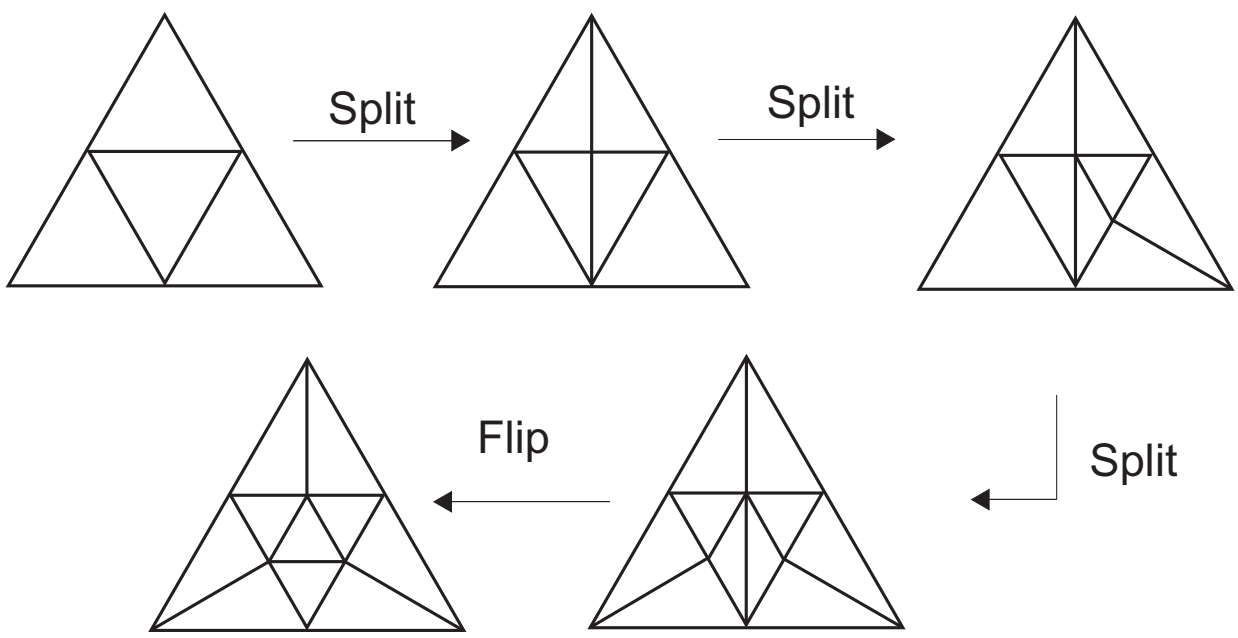

Figura 5.3: Subdivisão 1 para 4 subdividindo faces adjacentes

Como o operador de subdivisão 1 para 4 pode ser decomposto em operações estelares elementares, então este operador também é um operador estelar, desta forma, como descrito no capítulo 4, este operador não altera a topologia da superfície inicial durante o processo de subdivisão. 


\section{2 \\ Subdivisão Global}

$\mathrm{Na}$ subdivisão global, todas as faces da malha são recursivamente subdivididas aplicando o refinamento 1 para 4 descrito na seção anterior. A cada refinamento da malha $M^{i}$ é aplicado um algoritmo de busca em largura que se baseia nos operadores de Edge Split e Edge Flip como ilustrado na Figura 5.3. Naturalmente todas as referências para vértices, arestas e faces se baseiam nos seus respectivos corners.

Uma dificuldade do algoritmo de subdivisão é que ao aplicar o refinamento 1 para 4 em um triângulo, os seus vizinhos são subdivididos para evitar a criação de vértices $T$, o que implica em um gerenciamento dos estados das faces da malha durante o processo de subdivisão para garantir que a cada refinamento da malha $M^{i}$ todas as faces sofram apenas uma subdivisão 1 para 4 . Para este controle o algoritmo requer a utilização de vetores boleanos, facesVisitadas e facesProcessadas, de dimensão igual ao número de triângulos da malha refinada. $\mathrm{O}$ primeiro diz se a face sofreu o seu primeiro Edge Split, vindo de uma face vizinha, e o segundo indica se todas as arestas da face já foram subdivididas.

Uma outra dificuldade gerada pelo algoritmo mostrado na Figura 5.3 é o registro de qual aresta deve ser aplicado o operador de Edge Flip após todas todas as arestas da face terem sido subdivididas. Note que a aresta que sofre esta operação é sempre a correspondente a primeira aresta a dividir a face.

A seguir segue uma ideia geral de um algoritmo proposto.

1. Inicializar o vetores facesVisitadas e facesProcessadas .

2. Aplicar o operador de Edge Split em uma aresta correspondente a um corner inicial $c_{0}$ qualquer da malha. Esta operação gera o primeiro par vértice e aresta $(v, e)$ que deve ser colocado na fila $Q$, onde $v$ é o novo vértice gerado e $e$ é a aresta que subdiviu a face oposta. Guarde uma referência para a aresta $e_{0}$ que dividiu a face que contém o corner inicial e marque as novas faces geradas como visitadas.

3. Enquanto a fila $Q$ não for vazia, faça:

(a) Retire o primeiro par de corners correspondentes do vértice $v$ e aresta $e$ da fila $Q$.

(b) Processe o vértice $v$, subdividindo todas as faces de sua estrela que ainda não foram processadas. Cada aresta subdividida neste processo, gera um novo par $(v, e)$ deve ser colocado na fila $Q$. Cada face da estrela de $v$ é marcada como processada e as opostas 
como visitadas. Se uma face oposta já tiver sido visitada, então as faces geradas por sua subdivisão são marcadas como processadas.

(c) Aplique o operador de Edge Flip na aresta $e$.

4. Ao final da busca, aplicar o operador de Edge Flip na aresta $e_{0}$.

A Figura 5.4 illustra o funcionamento deste algoritmo. As arestas em destaque são aquelas as quais o operador de Edge Flip deve ser aplicado após cada vértice ser processado.

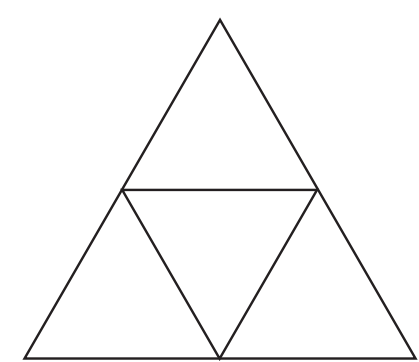

a)

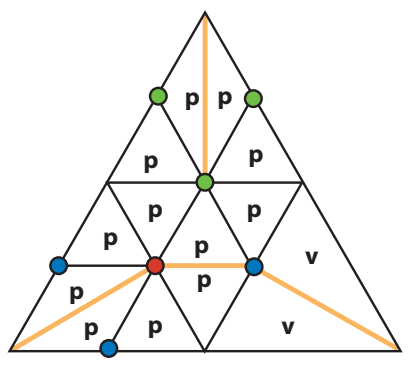

d)

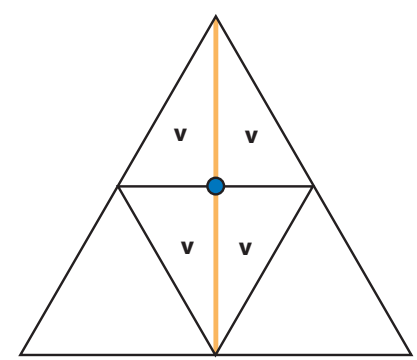

b)

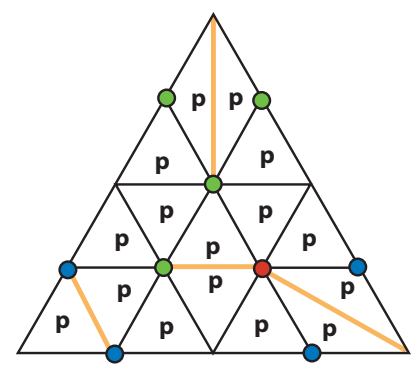

e)

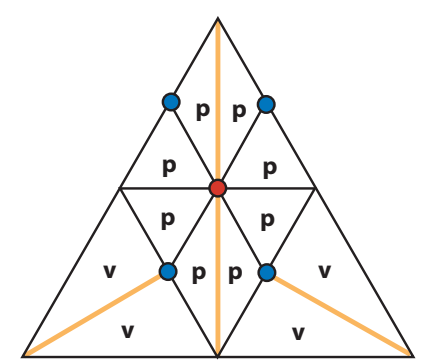

C)

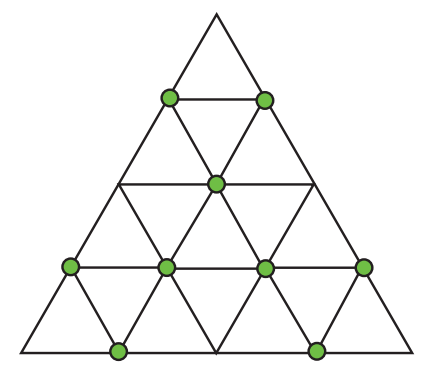

f)

Vértices na fila

$p=$ processada

Vértice sendo processado

$\mathrm{v}=$ visitada

Figura 5.4: a) Malha inicial a ser subdividida. b-e) Os pares de cornes $(v, e)$ são retirados da fila e as faces em suas estrelas que ainda não foram processadas são subdivididas. Ao processar um vértice, as faces de sua estrela são marcadas como processadas e as faces opostas são marcadas como visitadas. Após subividir toda estrela do vértice, o operador de Edge Flip é aplicado sobre a aresta oposta ao corner e. f) Malha refinada.

No Algoritmo 10 apresentamos uma implementação para o algoritmo da Figura 5.4 em Corner Table. O Algoritmo 10 usa o Algoritmo 11 para aplicar o operador de Edge Split sobre as arestas e realizar as atualizações necessárias, enquanto o Algoritmo 10 é responsável pela busca em si. 


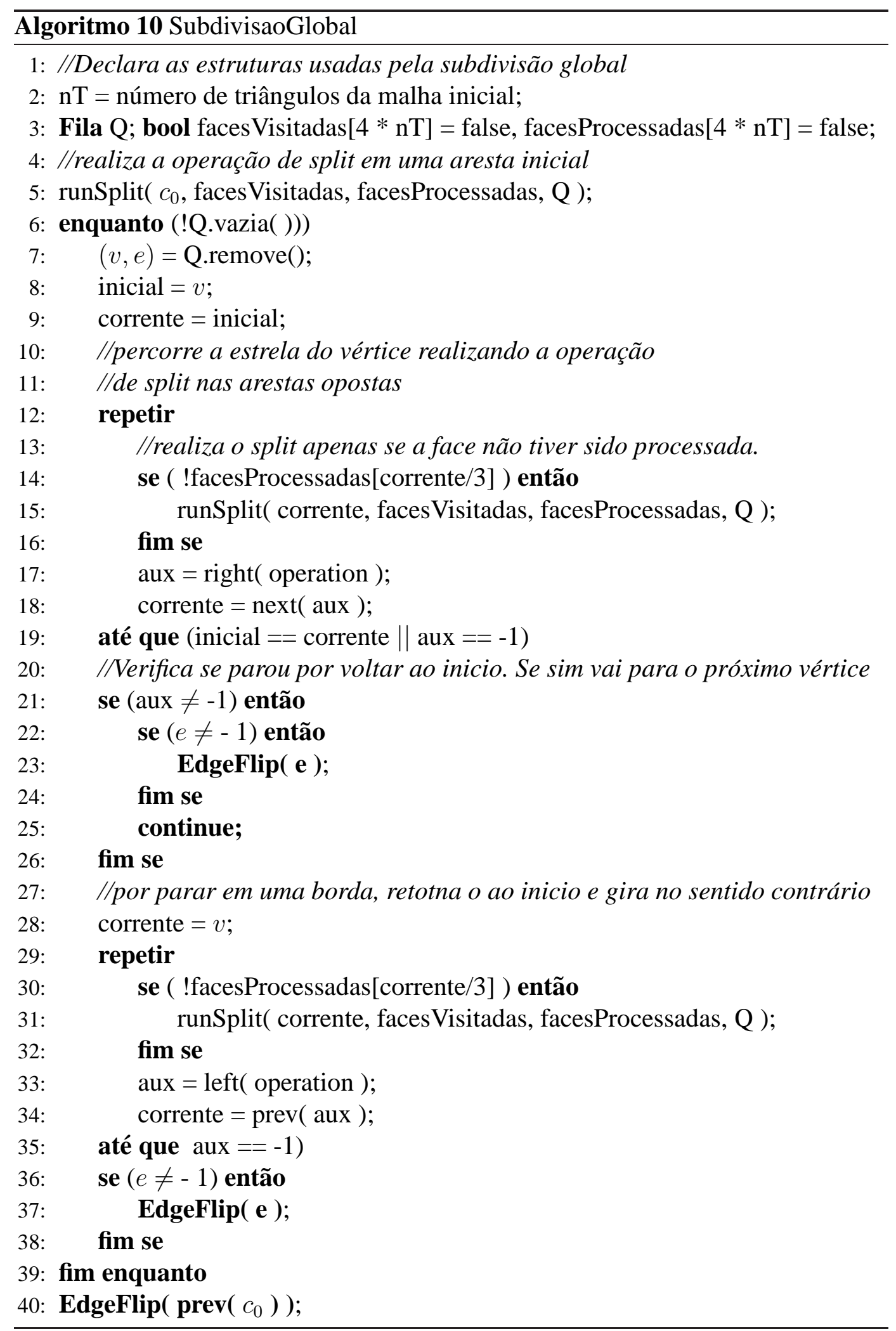

Note que ao aplicar o operador de Edge Split, o corner do próximo vértice a entrar na fila é sempre o next do corner corrente, e a aresta a qual será aplicado o operador de Edge Flip após o vértice ser processada será dada pelo previous do opposite do corner corrente. A convenção para o operador de Edge Split definida no capítulo 4 garante que mesmo para as faces opostas o corner oposto à aresta a 
$\overline{\text { Algoritmo } 11 \text { runSplit }}$ aplicado.

Entrada: facesVisitadas: vetor que diz se uma face já foi visitada.

Entrada: facesProcessadas: vetor que diz se uma face já foi processada.

Entrada: $Q$ : fila com os corners dos próximos vértices a serem processados e as arestas as quais o operador de Edge Flip deve ser aplicado após cada vértice ser processado.

1: //marca a face corrente e a nova face gerada como processadas

2: size $=$ número de triângulos da malha corrente

3: facesProcessadas[ corner / 3] = true;

4: facesProcessadas[size $]=$ true;

5: opposite $=\mathrm{O}[$ corner $]$;

6:

7: //Aplica o operador de Edge Split

8: EdgeSplit( corner );

9:

10: //Salva um corner do vértice gerado para ser inserido na fila

11: novoVertice $=\operatorname{next}($ corner $)$;

12: arestaParaFlip $=-1$;

13: se (opposite $\neq-1$ ) então

14:

15: $\quad / / S e$ a face oposta já foi visita, então marca a face oposta e nova face

16: $\quad / / g e r a d a$ a partir dela como processadas

17: $\quad$ se (faces Visitadas[opposite / 3]) então

18: $\quad$ facesProcessadas[opposite / 3] = true;

19: $\quad$ facesProcessadas $[$ size +1$]=$ true;

20: $\quad$ senão

21: $\quad$ /Como a face ainda nao foi visitada, a nova que cruza face

22: $\quad$ /lé aquela a qual o operador de Edge Flip deve ser

23: $\quad$ /laplicado após o vértice ser processado.

24:

25: arestaParaFlip $=\operatorname{prev}($ opposite $)$;

\section{fim se}

26:

27: $\quad$ /Marca a face oposta e a face gerada a partir dela como visitadas

28: $\quad$ facesVisitadas[opposite / 3] = true;

29: $\quad$ facesVisitadas $[$ size +1$]=$ true;

\section{0: fim se}

31: //Insere o par $(v, e)$ na fila

32: Q.insere( (novoVertice, arestaParaFlip)) ;

33:

ser "flipada"continua oposto à mesma aresta mesmo após as outras duas arestas da face serem subdivididas. Isto é ilustrado na Figura 5.6. Note ainda que, o operador de Edge Flip muda corners de posição, o que poderia mover o corner associado ao vértice enfileirado de posição, no entanto essa referência nunca é perdida pois o corner enfileirado continua associado ao mesmo vértice. Como descrito no capítulo 


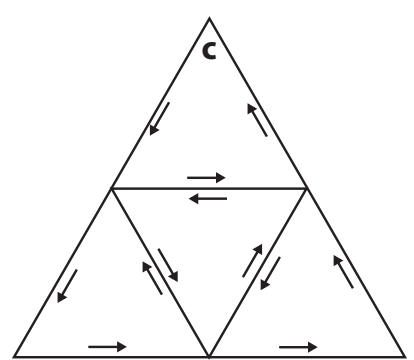

a)

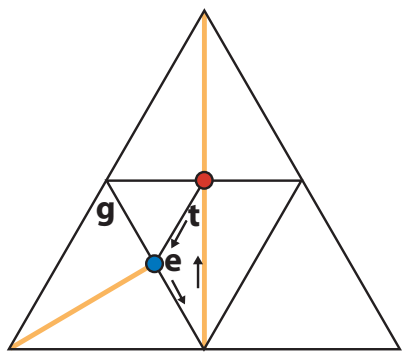

C)

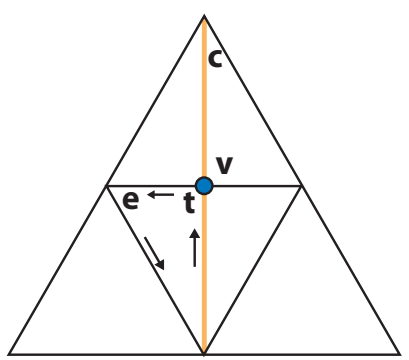

b)

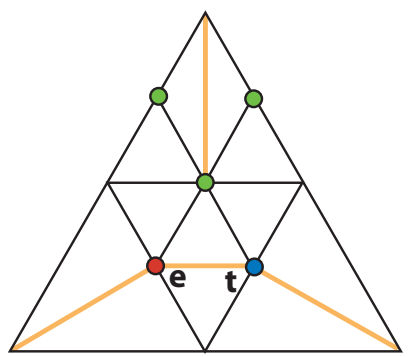

d)

Figura 5.5: a) Malha inicial. b) O operador de Edge Split é inicialmente aplicado a partir do corner c. O par $(v, e)$ é colocado na fila Q. c) Ao subdvidir as faces na estrela do vértice $V[v]$, o operador de Edge Split é aplicado a partir do corner $t$ e o par $(e, g)$ é colocado na fila Q. d) Após processar o vértice $V[v]$, o operador de Edge Flip é aplicado a patir do corner $e$. Apenas o corner $t=\operatorname{prev}(e)$ associado ao vértice que já foi processado muda de vértice, logo os outros corners da face que podem já está na fila associados com os seus vértices continuam associados com os mesmos vértices.

4 e ilustrado na Figura 5.5, ao aplicar aplicar o operador de Edge Flip a partir de um corner e, o único corner da face que muda de vértice é o prev(e), que está associado ao vértice que está sendo processado. Logo, a única referência perdida é para o vértice $V[v]$, que já foi retirado da fila. A mesma propriedade é valida para a face oposta.

Na Figura 5.6, a face inicial é primeiro subdividida a partir do corner 0, logo o corner 1 é colocado na fila para que as faces na estrela do vértice $V[1]$ sejam subdivididas posteriormente, e o corner 2 é guardado para que ao final o operador de Edge Flip seja aplicado a partir dele.

Para realizar a subdivisão global, a Corner Table em seu estado original original é suficiente, logo a tabela $C$ não é necessária. Com isso, um montante de memória é economizado sem nenhuma perda em eficiência. Além disso o Algoritmo 10 usa uma fila como estrutura auxiliar para realizar a busca na malha e dois vetores de boleanos, que juntos são responsáveis por um consumo de memória equivalente a 2 bits por face refinada. 


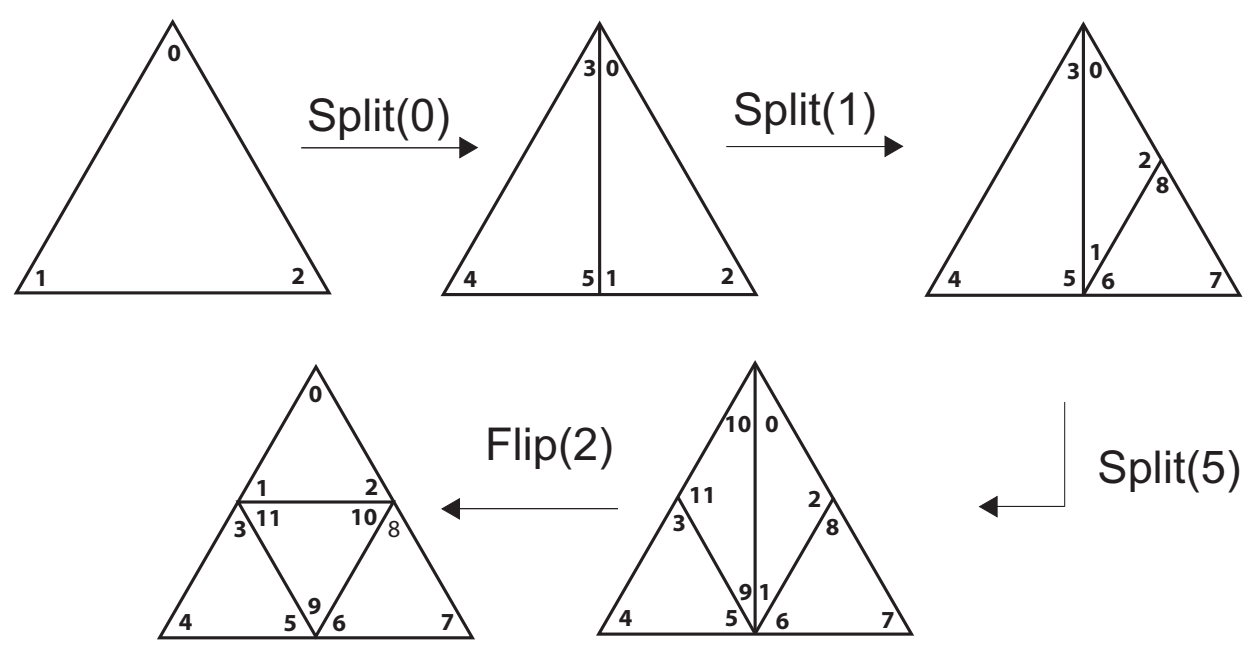

Figura 5.6: Exemplo da aplicação do algoritmo de subdivisão em um triângulo usando Corner Table. Sequência de aplicação dos operadores: EdgeSplit( 0 ), EdgeSplit( 1 ), EdgeSplit( 5 ) e EdgeFlip( 2 )

\section{3}

\section{Subdivisão Local}

Na subdivisão local apenas um subconjunto das faces é subdividido. Como mostrado no capítulo 2 isso pode gerar algumas inconsistências. O esquema de subdivisão adaptativa proposto por (Pakdel and Samavati, 2007) resolve estas inconsistências, por esse motivo adotamos o método de subdivisão incremental como o método de subdivisão adaptativa a ser implementado.

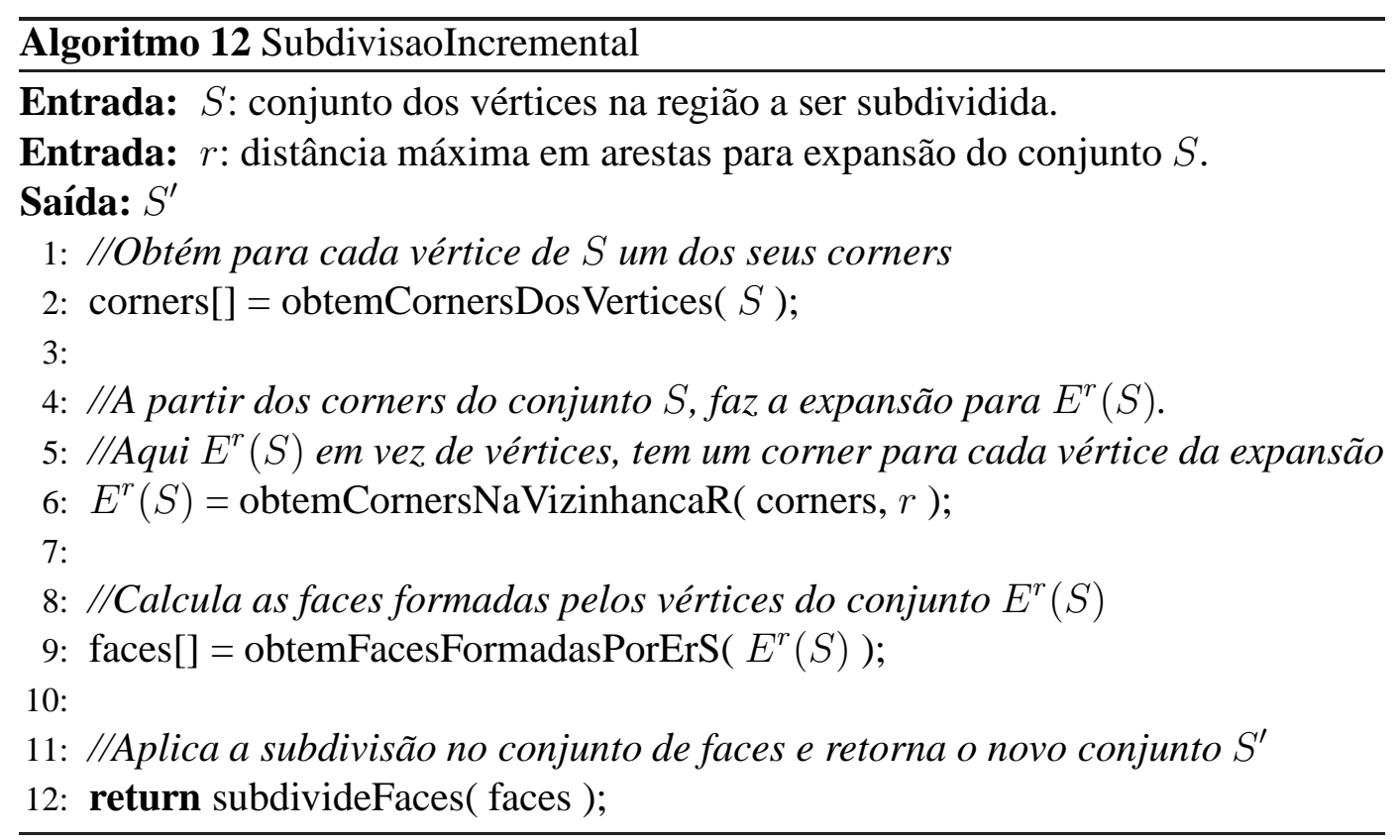

A entrada para o algoritmo de subdivisão incremental é um conjunto $S$ de vértices de uma área selecionada para subdivisão. Em seguida esse conjunto é expandido para um novo conjunto $E^{r}(S)$ que contém todos os vértices de $S$ e mais 
aqueles que distam ao menos $r$ arestas de $S$, e então a região que contém estes vértices é subdividida. No entanto o operador de subdivisão 1 para 4 é aplicado sobre faces da malha, logo alguns passos anteriores à subdivisão das faces de fato devem ser efetuados. O Algoritmo 12 realiza os passos necessários para subdividir adaptativamente a malha de acordo com a subdvisão incremental e a seguir cada passo é explicado com mais detalhes.

\subsection{1}

\section{Obtenção de corners de um conjunto de vértices}

A implementação da função que obtém um corner para cada vértice da linha 2 do Algoritmo 12 depende se a Corner Table implementada tem a tabela $C$ ou não. Se a tabela $C$ existir a obtenção destes cornes tem um custo linear com o número de vértices, uma vez que basta consultar esta tabela, caso contrário, a obtenção de cada corner tem tempo linear, logo o custo para obtenção de todos os corners é $O\left(n_{t} n_{v}\right)$, onde $n_{v}$ é o número de vértices e $n_{t}$ é o número de triângulos da malha corrente. A implementação desta rotina, usando a Corner Table, é mostrada no Algoritmo 13.

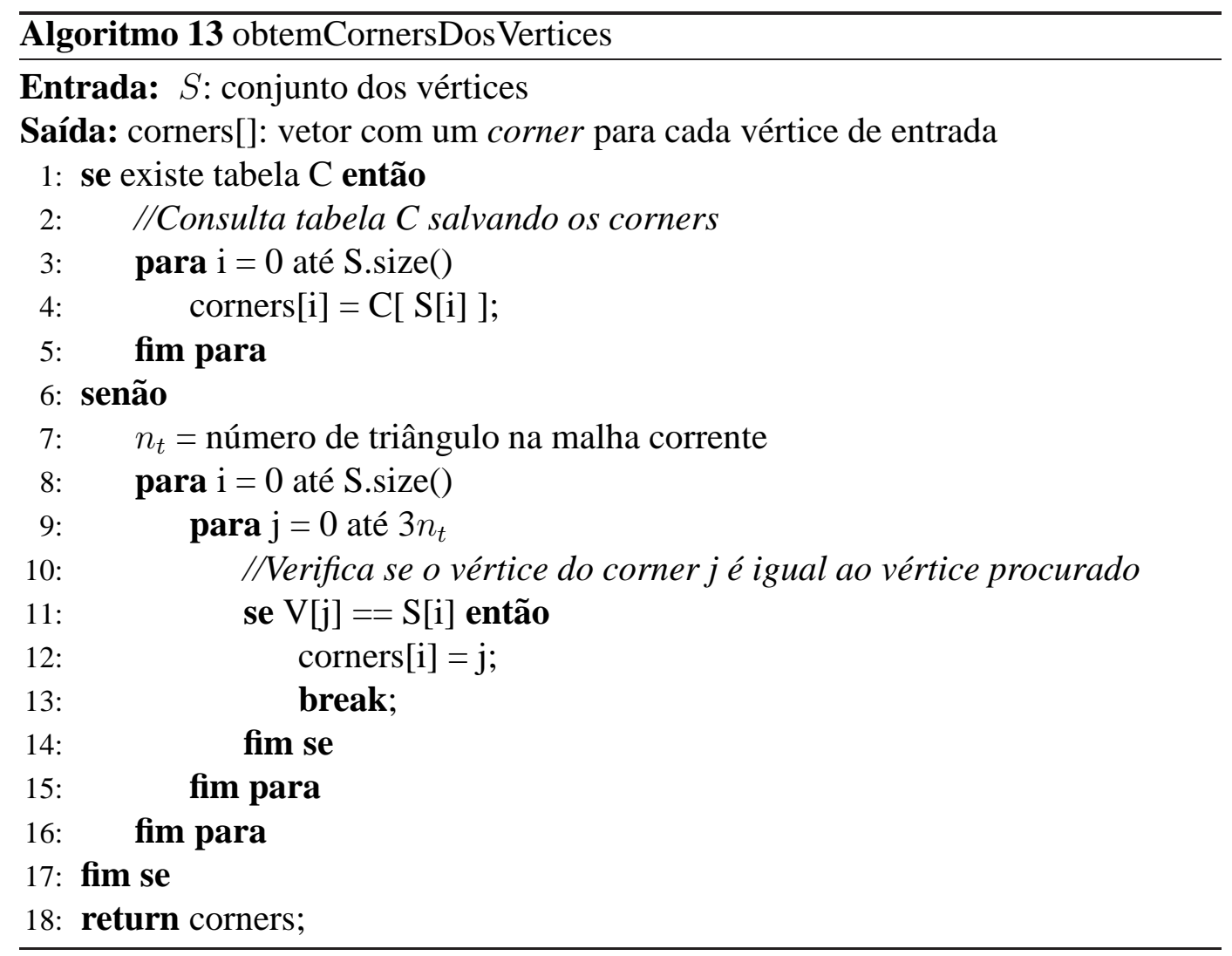

Note que algumas aplicações requerem que a entrada seja um conjunto de faces. Desta forma o algoritmo para obter os corners dos vértices é mais simples e não requer a tabela $C$ (ver Algoritmo 14).

Apesar de mais lenta em buscas topológicas que tem como entrada os vértices, a implementação da Corner Table sem a tabela $C$ é indicada para aplicações que 


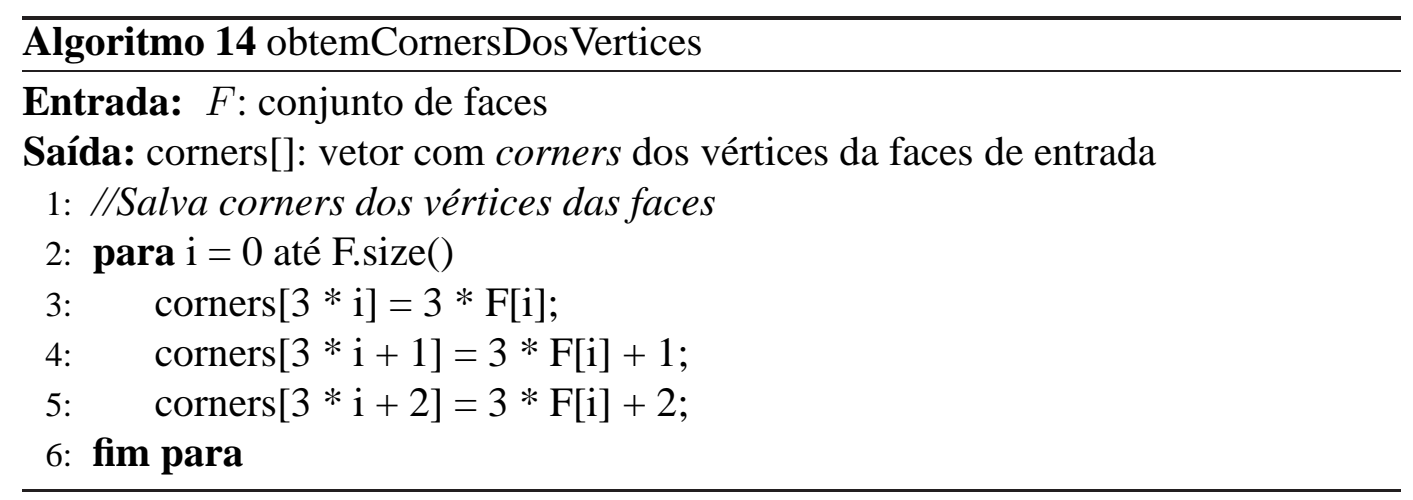

tenham limitação no uso de memória física ou aquelas que as regiões a serem subdivididas não tenham como entrada um conjunto muito grande de vértices.

Este é o único passo onde a tabela $C$ se faz necessária para aumentar a eficiência na realização da subdivisão incremental na Corner Table. Nos próximos passos usaremos apenas as tabelas $V$ e $O$.

\section{3 .2}

\section{Cálculo da $r$-vizinhança}

O segundo passo para realizar a subdivisão incremental é fazer a expansão do conjunto $S$ para um novo conjunto $E^{r}(S)$. Esse novo conjunto possui todos os vértices de $S$ e mais aqueles que distam ao menos $r$ arestas de algum vértice de $S$. Esta expansão é basicamente uma busca em largura a partir do conjunto $S$ que deve ser interrompida quando alcançar vértices que distam mais que $r$ arestas de $S$.

O Algoritmo 15 calcula a expansão de $S$, gerando um novo conjunto $E^{r}(S)$ que por sua vez tem um corner para cada vértice da $r$-vizinhança. O Algoritmo 15 usa o Algoritmo 17 para fazer a expansão a partir de um determinado vértice, incluindo seus vértices vizinhos no conjunto $E^{r}(S)$. Por sua vez, o Algoritmo 17 usa o Algoritmo 16, para que a partir de um corner $c$ se obtenha um vetor com um corner para cada vértice na estrela do vértice $V[c]$.

O Algoritmo 15 realiza a expansão do conjunto $S$ para um novo conjunto $E^{r}(S)$ usando uma fila e um vetor de inteiros como estruturas auxiliares. A fila é usada para armazenar os corners dos próximos vértices a serem expandidos na busca em largura, já o vetor de inteiros é usado para armazenar a menor distância ao conjunto $S$ para cada vértice. À medida que os vértices são alcançados as suas distâncias são atualizadas e seus vizinhos, se não estiverem a uma distância para $S$ maior que $r$, são adicionados na fila. Note que por se tratar de uma busca em largura, no primeiro momento em que um vértice fora do conjunto $S$ é alcançado, a distância corrente já é a sua menor distância.

O Algoritmo 16 obtém um corner para cada vértice na estrela de um determinado vértice. A partir de um corner, o algoritmo gira em um sentido na 


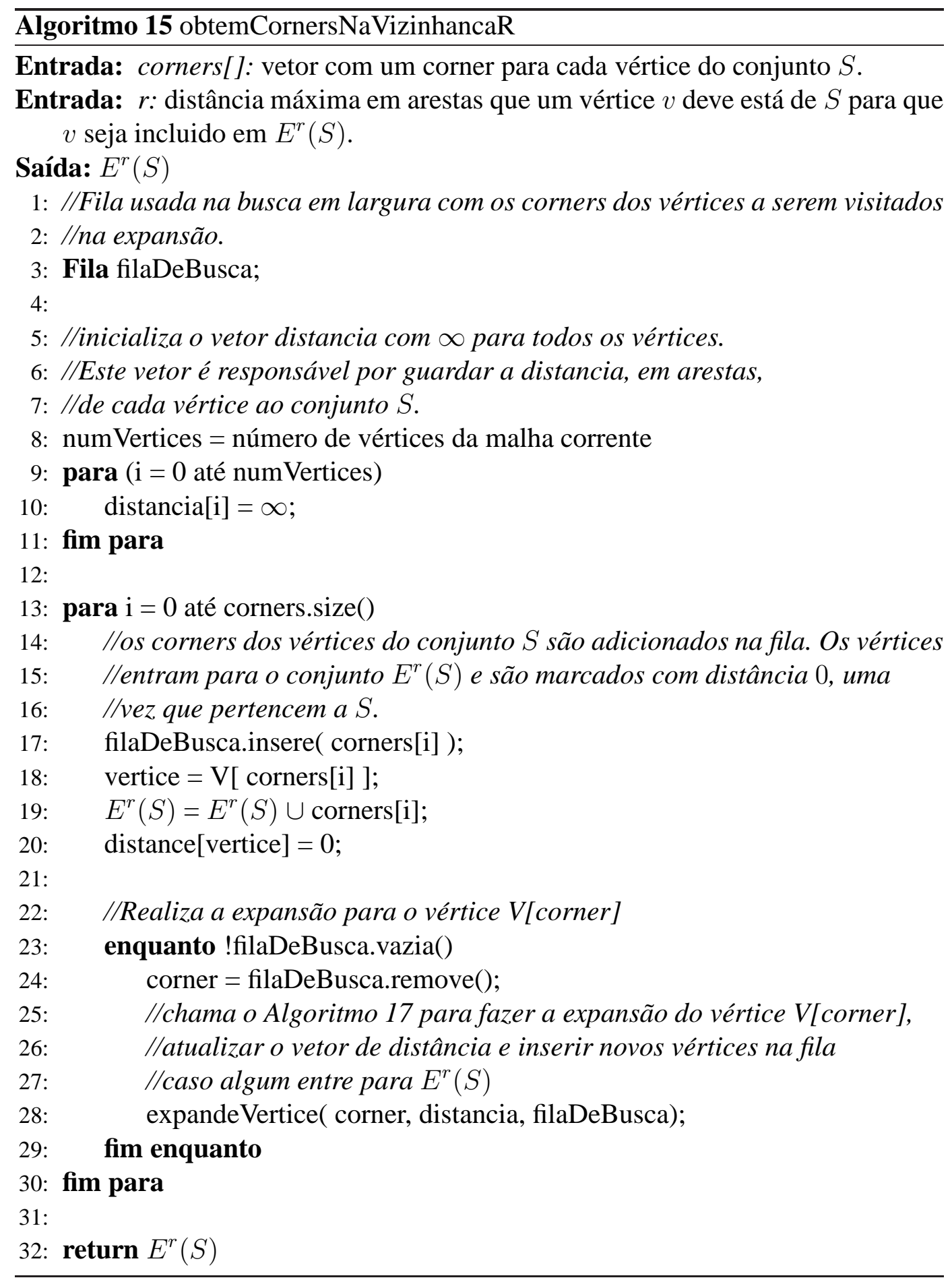

estrela de um determinado vértice obtendo os corners dos vértices vizinhos. O algoritmo pode se deparar com o bordo da superfície, neste caso o algoritmo retoma a partir do corner inicial e gira em outro sentido, novamente obtendo os corners dos vértices vizinhos até se deparar mais uma vez com o bordo da superfície.

O Algoritmo 17 realiza a expansão de um determinado vértice $v$ atualizando os vetores de distância para os vizinhos de $v$ e a fila usada para fazer a busca em largura. O algoritmo recebe um corner $c$, consulta os vértices na estrela de $V[c] \mathrm{e}$ atualiza a distância destes vértices caso o caminho até $S$ seja menor passando por 


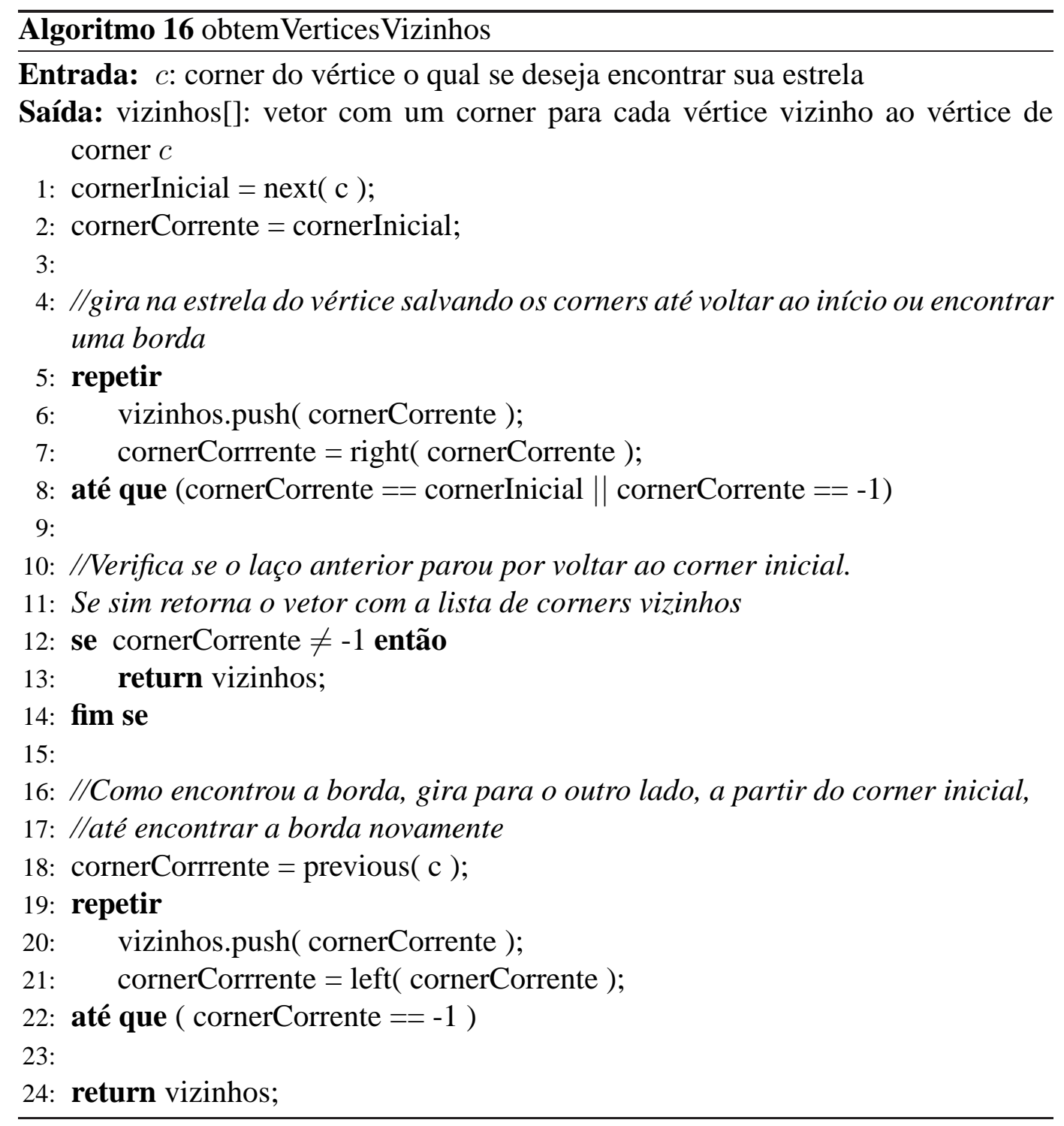

$V[c]$. Neste caso, os corners destes vértices são adicionados na fila, para que seus vizinhos também sejam consultados posteriormente. Além disso, um corner para cada um destes vértices é adicionado ao conjunto $E^{r}(S)$.

Note que o vetor distância funciona como uma espécie de cache das buscas dos outros vértices já realizadas, uma vez que as operações da linha 19 a linha 27 do Algoritmo 17 só são executadas para um vértice se for descoberto um caminho mais curto para ele, evitando assim que estas operações sejam executadas várias vezes para o mesmo vértice. 


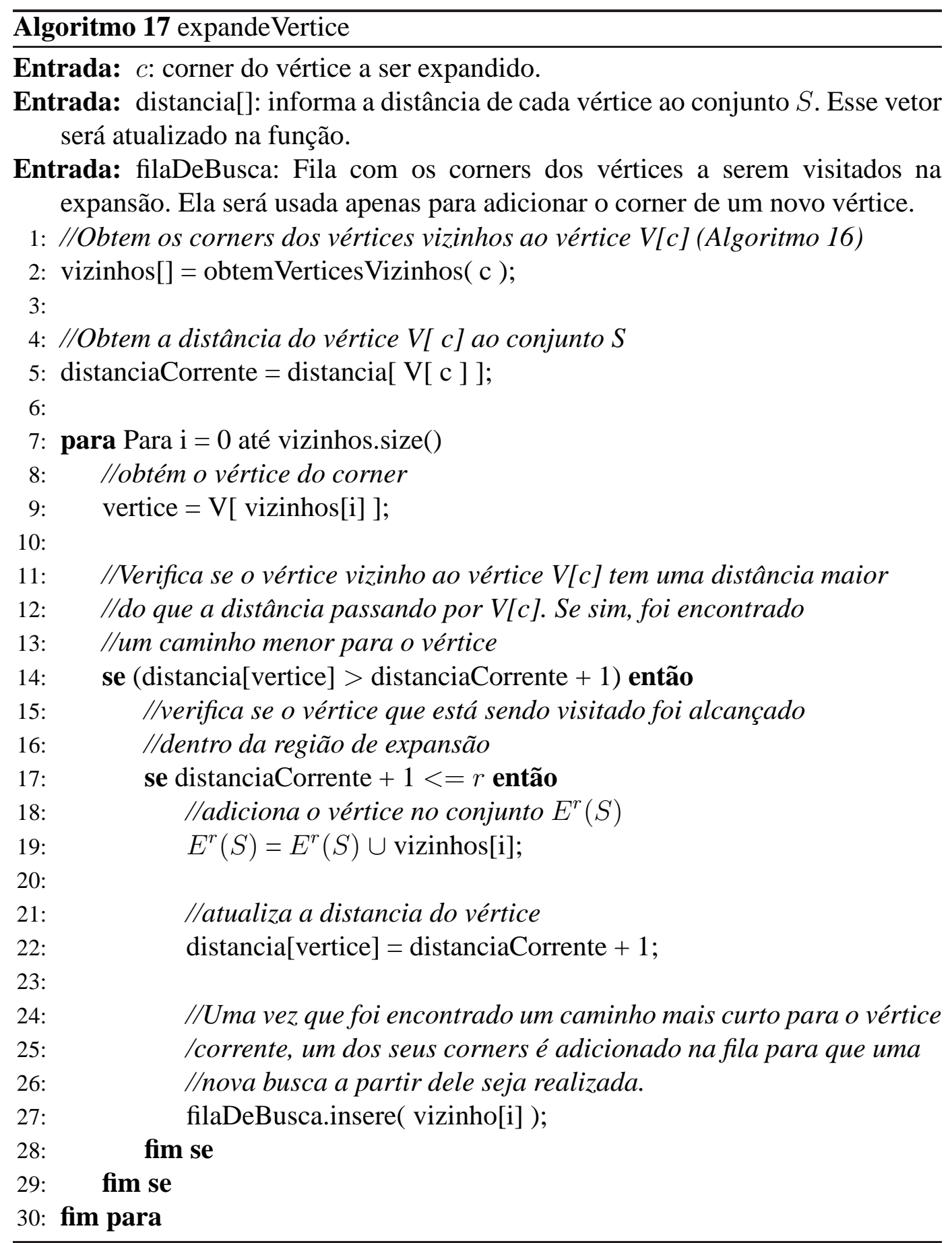

\subsection{3}

\section{Calculando as faces a partir de $E^{r}(S)$}

Uma vez que o operador de subdivisão 1 para 4 é aplicado sobre as faces da malha triangular, um terceiro passo é necessário para calcular quais as faces são formadas com os vértices do conjunto $E^{r}(S)$.

O Algoritmo 18 recebe o conjunto $E^{r}(S)$ e retorna as faces formadas por seus vértices. 


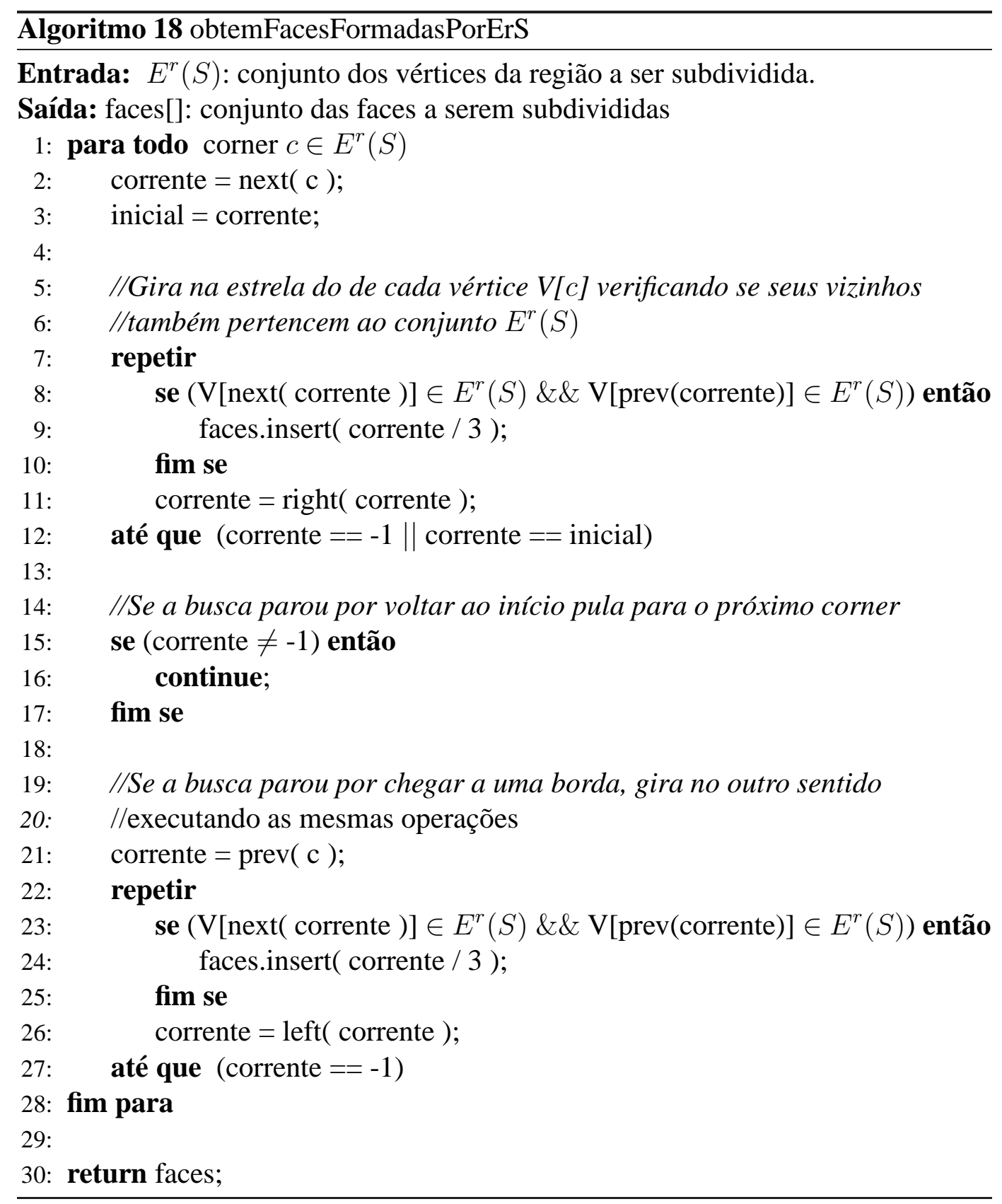

O Algoritmo 18 verifica para cada vértice dos corners em $E^{r}(S)$ se seus vértices vizinhos, dentro da mesma face, também pertecem a $E^{r}(S)$. Para cada corner $c \in E^{r}(S)$, o algoritmo gira na estrela do vértice $V[c]$ verificando se next(c) e $\operatorname{prev}(c)$ também pertecem a $E^{r}(S)$ (ver Figura 5.7), em caso a afirmativo a face $f=\lfloor c \div 3\rfloor$ é adicionada no conjunto das faces que serão subdivididas posteriormente. $\mathrm{O}$ algoritmo pode se de deparar com o bordo da superfície. Nesse caso, o algoritmo retoma a busca a partir do corner inicial, mas girando no sentido oposto desta vez. 


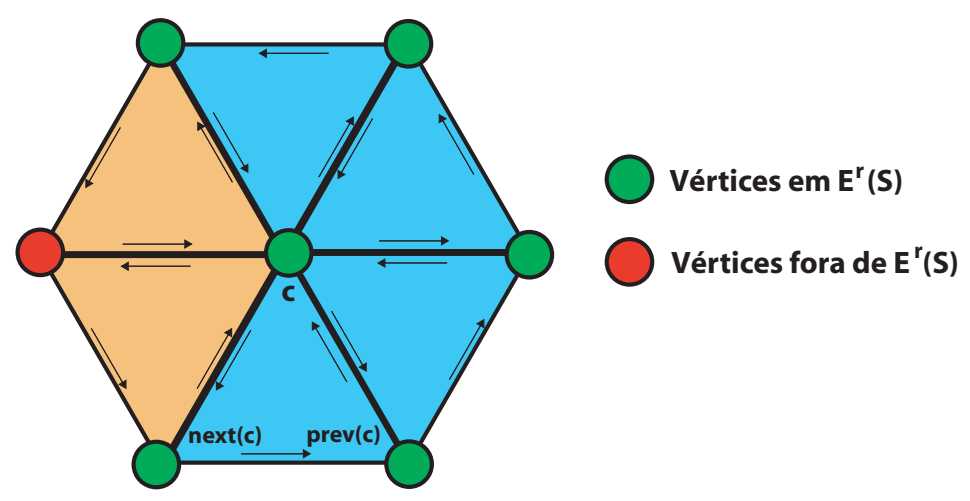

Figura 5.7: Ilustração do funcionamento do Algoritmo 18. As faces em azul são aquelas que serão subdivididas posteriormente.

\subsection{4}

\section{Subdivisão da região expandida}

Com o conjunto das faces a serem subdivididas calculado, o último passo da subdivisão adaptativa pode ser realizado. Neste passo as faces calculadas são subdivididas e para possibilitar vários níveis de subdivisão da mesma região, o novo conjunto $S^{\prime}$ é retornado. Lembrando que $S^{\prime}$ é o conjunto de vértices formado por $S$ mais os vértices criados sobre as arestas das faces formadas por $S$ (ver Figura 2.14).

O algoritmo para subdivisão adaptativa das faces selecionadas é bastante semelhante ao algoritmo de subdivisão global, no entanto como nem todas as faces são subdivididas, uma estrutura auxiliar é necessária para informar se uma face é subdividida ou não. Esta estrutura pode ser um vetor de boleano de tamanho $4 n_{t}$, onde $n_{t}$ é o número de triângulos da malha corrente, que informa em tempo constante se uma face é subdividida ou não, ou até mesmo uma árvore balanceada, onde os nós são os índices das faces a serem subdivididas. Note que ao subdividir uma face, a nova face gerada pela subdivisão deve ser colocada na árvore também. Desta forma, ao fim da subdivisão a árvore terá $4 n$ nós.

Se o número de faces a serem subdivididas for pequeno em relação ao número total de faces da superfície, a implementação usando uma árvore balanceada pode usar um montante de memória menor, no entanto o custo para determinar se uma face deve ser subdividida ou não é de $\log (n)$, onde $n$ é o número de faces a serem subdivididas.

O vetor de booleano pode usar um bit por face, já a árvore usa um inteiro de 32 bits por nó, logo se o fator determinante para escolha da estrutura auxiliar for o montante de memória usado durante a subdivisão, a árvore deve ser escolhida sempre que

$$
32(4 n)<4 n_{t}
$$




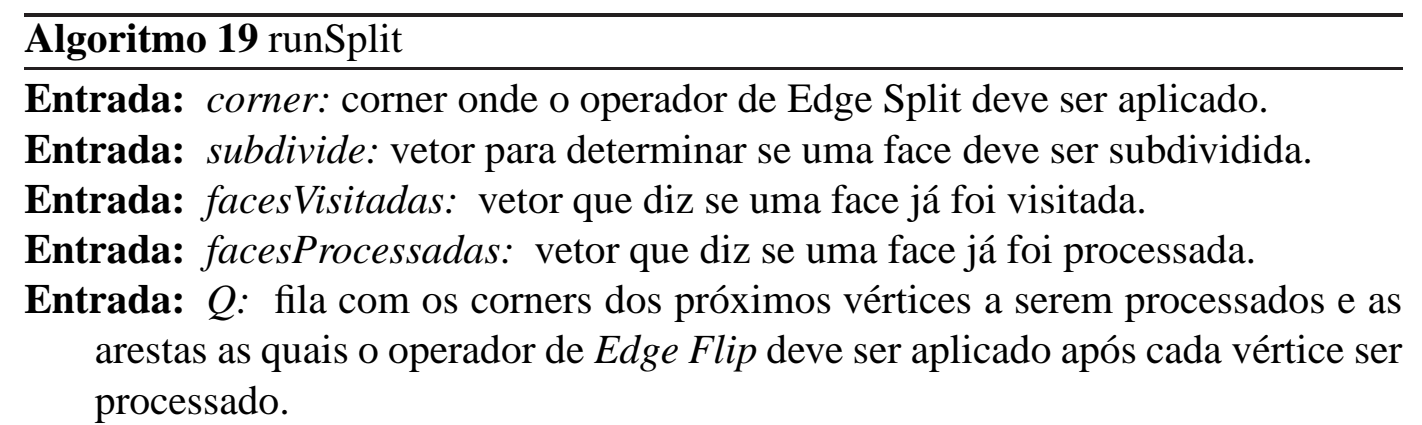

Entrada: $S$ : conjunto de vértices da região selecionada.

Entrada: $S^{\prime}$ : conjunto $S^{\prime}$ que deve ser atualizada.

1: size $=$ número de triângulos da malha corrente

2: $n_{t}=$ número de vértices da malha corrente

3:

4: //Verifica se o novo vértice será criado sobre uma aresta da região selecionada.

5: //Se sim adiciona o índice do vértice vértice ao conjunto $S^{\prime}$

6: se (V[next(corner) $] \in S \& \& \mathrm{~V}[\operatorname{prev}($ corner $)] \in S$ ) então

7: $\quad S^{\prime}=S^{\prime} \cup n_{t}$;

8: fim se

9:

10: facesProcessadas $[$ corner / 3] = true;

11: facesProcessadas [size $]=$ true;

12: opposite $=\mathrm{O}[$ corner $]$;

13:

14: //Aplica o operador de Edge Split

15: EdgeSplit( corner);

16:

17: //Salva um corner do vértice gerado para ser inserido na fila

18: novoVertice $=\operatorname{next}($ corner $)$;

19: arestaParaFlip $=-1$;

20: se (opposite $\neq-1$ ) então

21: //Salva corner oposto a aresta que deve ser "flipada".

22: $\quad$ se (subdivide[opposite / 3] \& \& !facesVisitadas[opposite / 3]) então

23: $\quad$ arestaParaFlip $=\operatorname{prev}($ opposite $)$;

24: $\quad$ fim se

25: $\quad$ //Se a face oposta já foi visita, então marca a face oposta e nova face

26: $\quad / / g e r a d a$ a partir dela como processadas

27: $\quad$ se (faces Visitadas[opposite / 3]) então

28: $\quad$ facesProcessadas[opposite / 3] = true;

29: $\quad$ facesProcessadas $[$ size +1$]=$ true;

30: fim se

31: $\quad$ //Marca a face oposta e a face gerada a partir dela como visitadas

32: $\quad \ldots$

33: fim se

34: //Insere o par $(v, e)$ na fila

35: Q.insere( (novoVertice, arestaParaFlip)) ; 
caso contrário, o vetor de booleano deve ser escolhido.

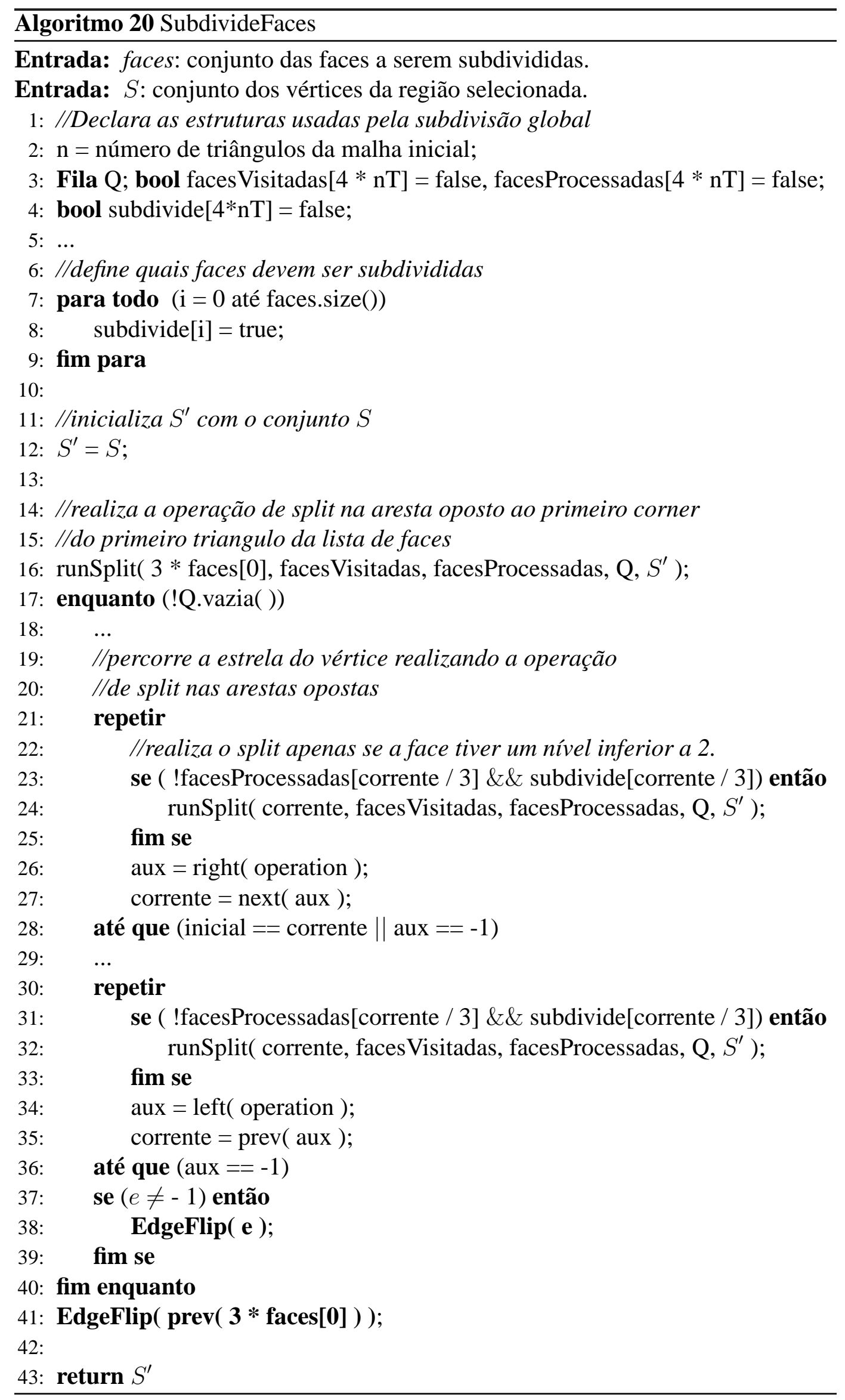


Os Algoritmos 19 e 20 implementam a subdivisão das faces selecionadas usando um vetor de booleano para determinar quais faces devem ser subdivididas. Pela semelhança com os algoritmos 11 e 10, algumas linhas serão omitidas. Além disso, o Algoritmo 20 retorna o conjunto $S^{\prime}$. É importante retornar esse conjunto de vértices, pois é a partir dele que a subdivisão incremental deve ser aplicada para se produzir vários níveis de subdivisão em uma mesma região da superfície.

\section{4}

\section{Undo e Redo para Subdivisão}

Para algumas aplicações, como por exemplo modelagem, é importante fornecer uma forma de desfazer ou refazer um conjunto de passos. Por isso, provemos um mecanismo para realizar undo e redo para as operações de subdivisão.

Uma forma de realizar undo e redo nas operações de uma superfície, seria amarzenar uma superfície a cada modificação sofrida pela superfície corrente. No entanto, isto seria muito ineficiente no que diz respeito ao uso de memória. Uma forma eficiente seria pela aplicação das operações inversas às operações que produziram o estado corrente da superfície.

Como mostrado na seção 5.1, as subdivisões global e adaptativa são realizadas através da aplicação dos operadores estelares Edge Split e Edge Flip e no capítulo 4 foi visto que o operador inverso ao Edge Split é o operador de Edge Weld, e o operador inverso ao Edge Flip é o próprio Edge Flip. Ainda no capítulo 4 foi mostrada a necessidade de haver uma implementação para reverter o operador de Edge Flip, que chamamos de Edge Flip Inverso. Baseado nisso e nas convenções que assumimos para implementar as operações, as figuras 5.8 e 5.9 ilustram a aplicação dos operadores inversos usados na subdivisão.

Como ilustrado na Figura 5.8, para reverter o efeito provocado pelo operador Edge Split aplicado em um corner $c_{0}$, basta aplicar o operador Edge Weld a partir do corner next $\left(c_{0}\right)$ e para reverter o efeito provocado pelo operador Edge Weld aplicado em um corner $c_{1}$, basta aplicar o operador Edge Split a partir do corner prev $\left(c_{1}\right)$. Já para reverter o efeito provocado pelo operador Edge Flip aplicado a partir de um corner $c_{0}$, basta aplicar o Edge Flip Inverso a partir do mesmo corner next $\left(c_{0}\right) \mathrm{e}$ para reverter o efeito provocado pela aplicação do Edge Flip Inverso a partir de um corner $c_{1}$, basta aplicar o operador de Edge Flip a partir do mesmo corner prev $\left(c_{0}\right)$.

O operador de Edge Weld remove um vértice e dois triângulos da malha, se o vértice removido não for do bordo da superfície, mas é desejável que a operação de undo matenha sempre as tabelas $V$ e $G$ contíguas, ou seja, não remova triângulos ou vértices no meio destas tabelas, de forma que se a malha tiver $n$ triângulos, estes estão gravados nas $3 n$ primeiras posições da tabela $V$ e da mesma forma a tabela $G$. Note que esta condição é automaticamente satisfeita, uma vez que ao aplicar o 

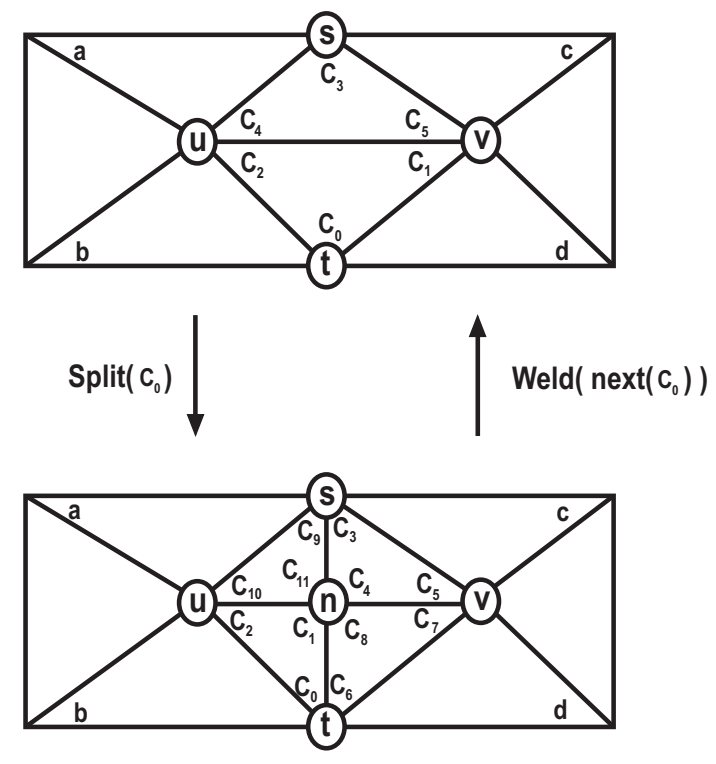

Figura 5.8: Inversão do operador de Edge Split pela aplicação do operador de Edge Weld e vice-versa.
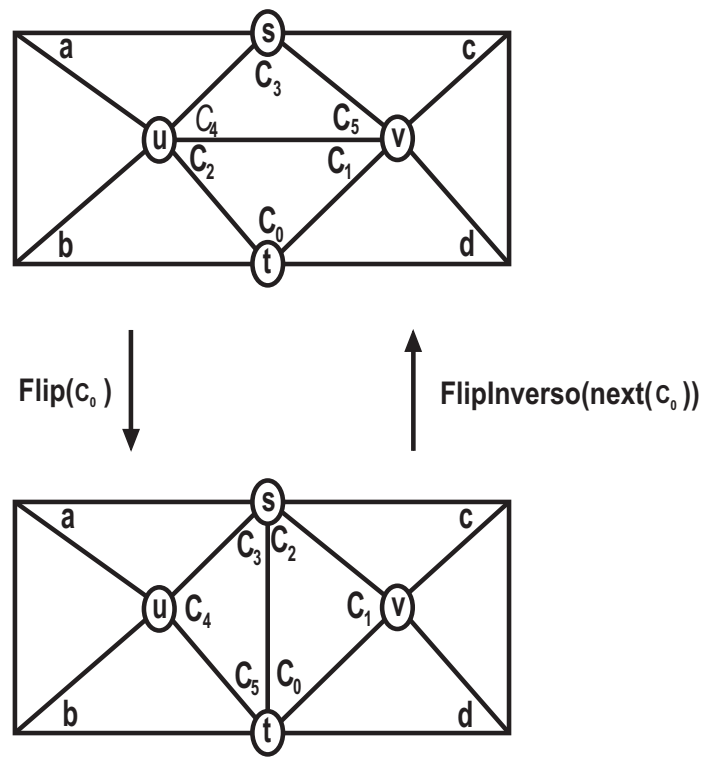

Figura 5.9: Invrsão do operador de Edge Flip pela aplicação do Edge Flip Inverso e vice-versa.

operador de Edge Split, este sempre posiciona os novos triângulos e o novo vértice ao final das tabelas $V$ e $G$ respectivamente, logo ao reverter uma sequência de passos o operador de Edge Weld sempre removerá vértices e triângulos do final de suas tabelas, mantendo assim as tabelas $V$ e $G$ sempre contíguas. Isso evita um passo extra para rearranjar estes vetores e nenhum montante de memória adicional para execução do mecanismo de undo/redo é requerido. 


\begin{tabular}{|c|c|}
\hline Operação & Operação Inversa \\
\hline EdgeSplit $(c)$ & EdgeWeld $($ next $(c))$ \\
\hline EdgeWeld $(c)$ & EdgeSplit $($ previous $(c))$ \\
\hline EdgeFlip $(c)$ & EdgeFlipInverso $($ next $(c))$ \\
\hline EdgeFlipInverso $(c)$ & EdgeFlip $($ prev $(c))$ \\
\hline
\end{tabular}

Tabela 5.1: Operações Inversas

A tabela 5.1 mostra as operações inversas que devem ser empilhadas para executar o mecanismo de undo/redo. 


\section{6 \\ Resultados}

Neste capítulo apresentamos alguns resultados da subdivisão global e adaptativa. Para avalizar a eficiência foram feitas medições de tempo para os dois algoritmos. Ambos também foram aplicados em alguns modelos para avaliar a malha resultante e qualidade de seus triângulos.

\section{1}

\section{Subdivisão Global}

Para avaliar a eficiência do algoritmo de subdivisão global, aplicamos recursivamente o algoritmo de subdivisão em uma malha triangular de uma superfície plana com bordo. Subdividimos recursivamente um triângulo onde os novos vértices criados pelo processo de subdivisão são sempre posicionados sobre o ponto médio das arestas. A Tabela 6.1 mostra o número de faces antes e depois de cada subdivisão o tempo de execução.

\begin{tabular}{|c|c|c|}
\hline $\begin{array}{c}\text { Número de faces antes } \\
\text { da subdivisão }\end{array}$ & $\begin{array}{c}\text { Número de faces } \\
\text { após a subdivisão }\end{array}$ & Tempo (segundos*) \\
\hline 1.024 & 4.096 & $<0.01$ \\
\hline 4.096 & 16.384 & $<0.01$ \\
\hline 16.384 & 65.536 & $<0.01$ \\
\hline 65.536 & 262.144 & 0.02 \\
\hline 262.144 & 1.048 .576 & 0.08 \\
\hline 1.048 .576 & 4.194 .304 & 0.38 \\
\hline 4.194 .304 & 16.777 .216 & 1.76 \\
\hline 16.777 .216 & 67.108 .864 & 5.74 \\
\hline
\end{tabular}

Tabela 6.1: Tempo para realizar a subdivisão global. *Core i5 3.10Ghz, 8GB RAM

Como segundo exemplo executamos uma subdivisão global sobre um icosaedro regular para gerar a malha de uma esfera (Figura 6.1). O icosaedro regular está mostrado na Figura 6.1(a). Nas Figuras de 6.1(b)-6.1(e) cada novo vértice gerado é posicionado sobre a superfície da esfera interpolante deste icosaedro. A Figura 6.1(f) mostra o icosaedro de 6.1(a) com o mesmo nível de subdivisão de 6.1(e), mas com os novos vértices posicionados usando Butterfly.

Na Figura 6.2, a subvisão global é usada para aproximar uma malha plana da função $z=\sin (\pi x) \cos y+0.1 \sin (2 \pi x)$. Em todos os outros exemplos de 
subdivisão global, o esquema Butterfly foi utilizado para posicionar os novos vértices.

A Figura 6.3, 6.4, 6.5, 6.6(a), 6.7 ilustram a subdivisão de um tetraedro, de uma estrela de 6 pontas, e dos modelos cow, bunny e bimba respectivamente.

Apesar do Butterfly modificado poder ser aplicado em uma malha de topologia arbitrária, o método assume uma certa regularidade nos triângulos da malha. De forma que a presença de triângulos muito finos causam efeitos indesejados, como ilustra a Figura 6.7, onde o triângulo fino destacado 6.7(a) causa uma dobra na superfície. 


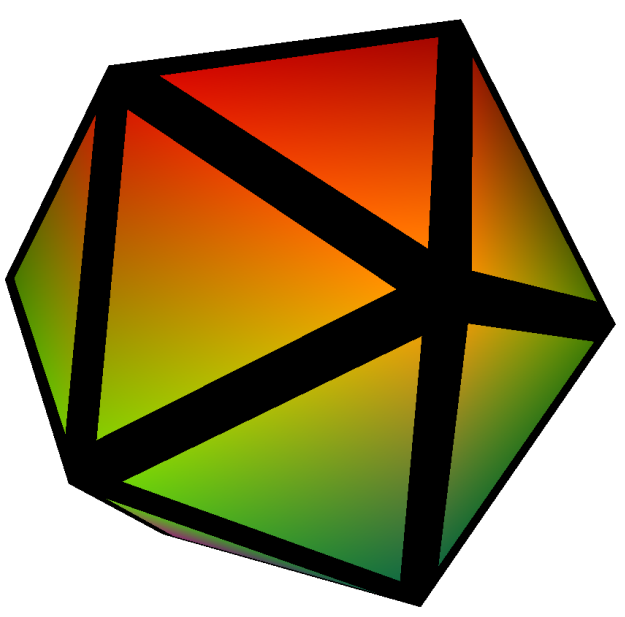

(a)20 faces

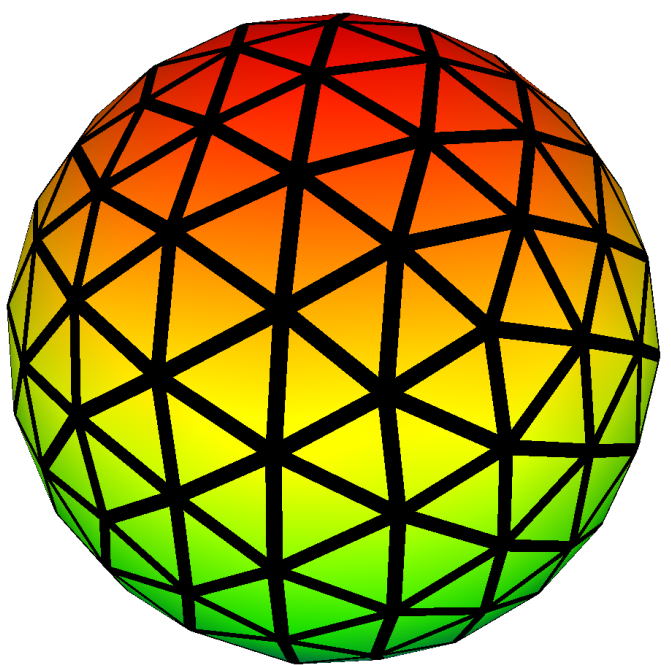

(c)320 faces

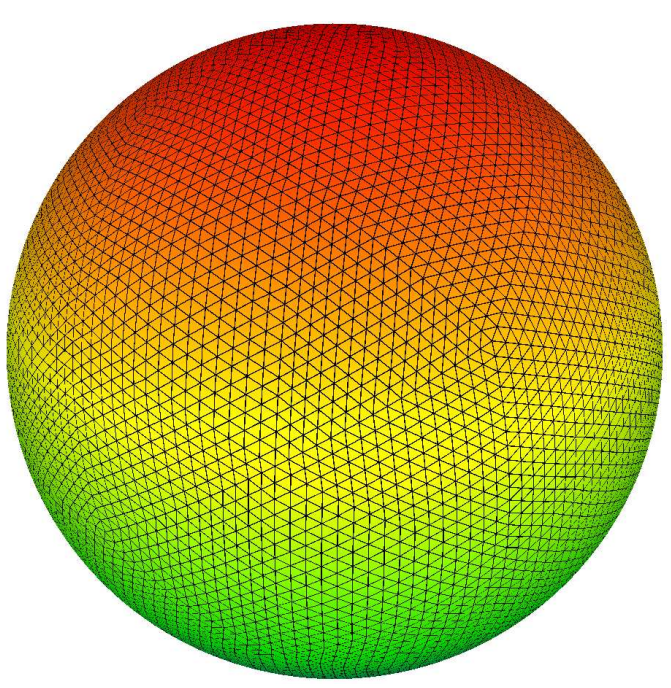

(e)20480 faces

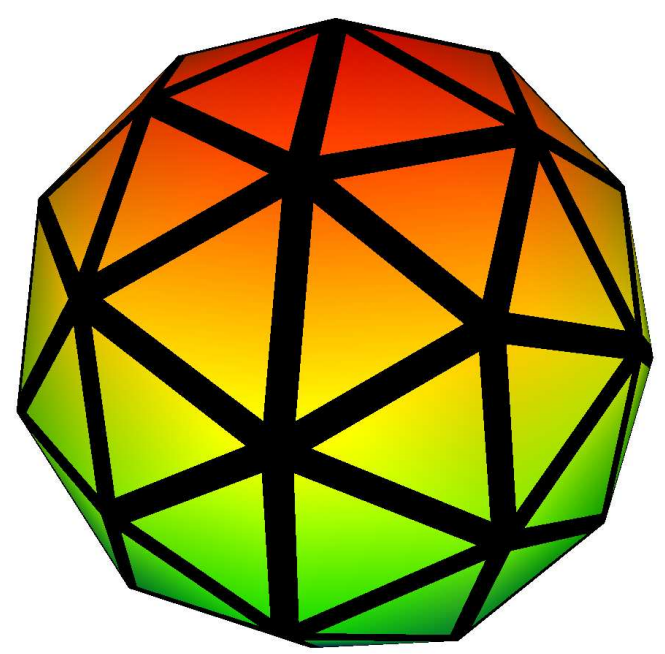

(b) 80 faces

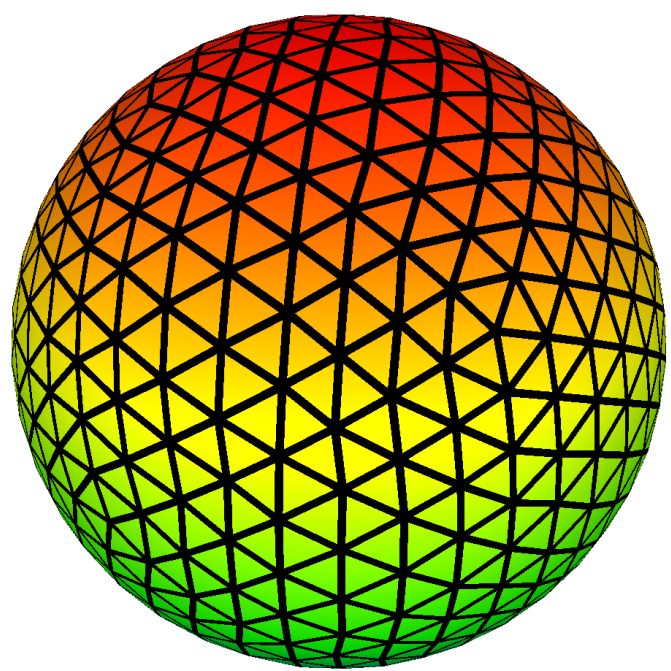

(d)1280 faces

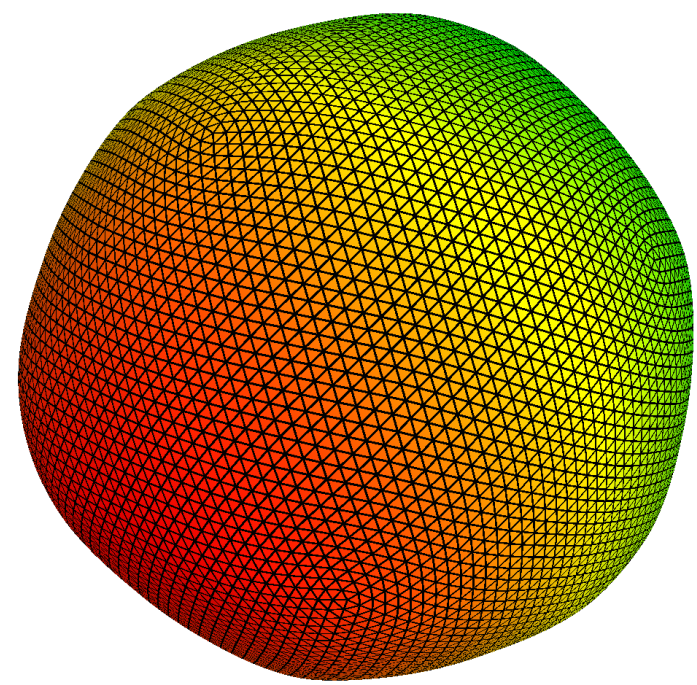

(f)Subdivisão do icosaedro regular usando Butterfly para posicionar os novos vértices. 20480 faces

Figura 6.1: Geração da malha de uma esfera através da subdivisão de um icosaedro regular 


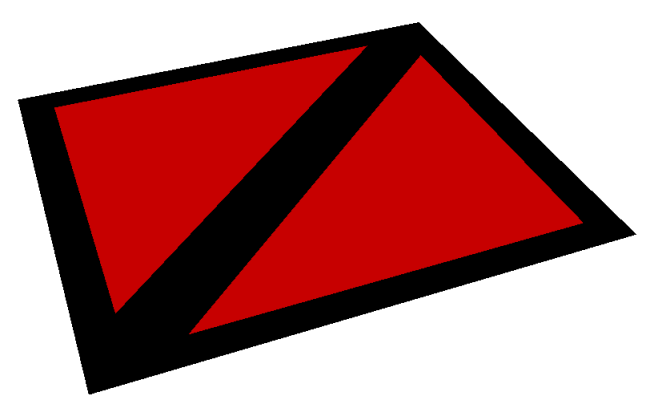

(a) 2 faces

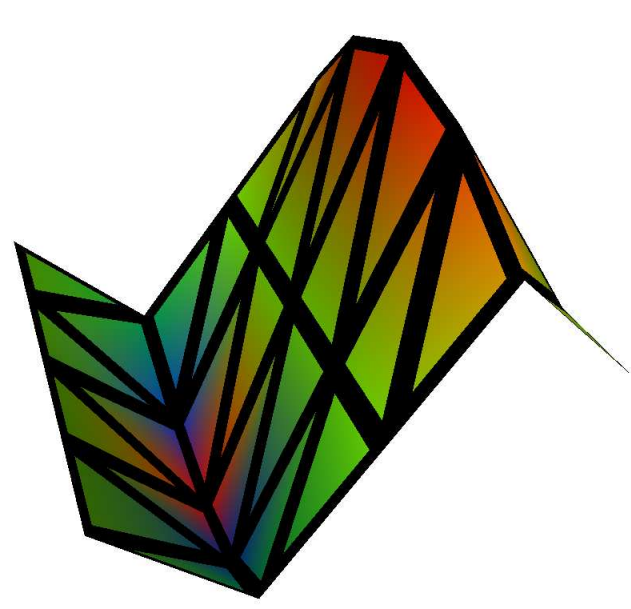

(c)32 faces

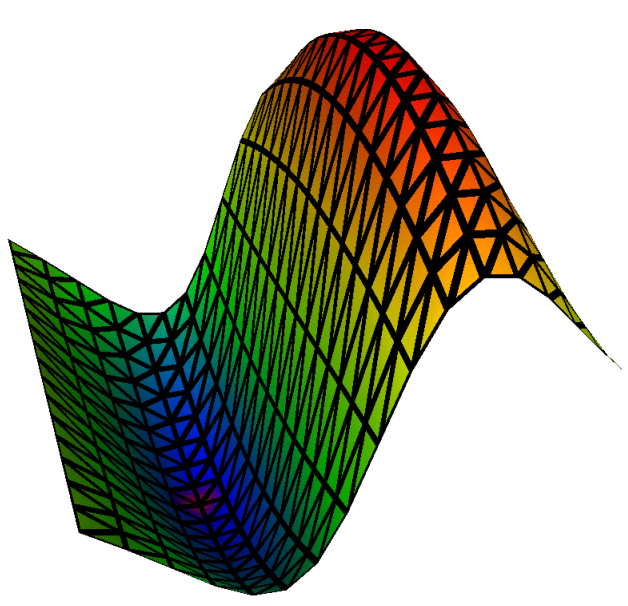

(e)2048 faces

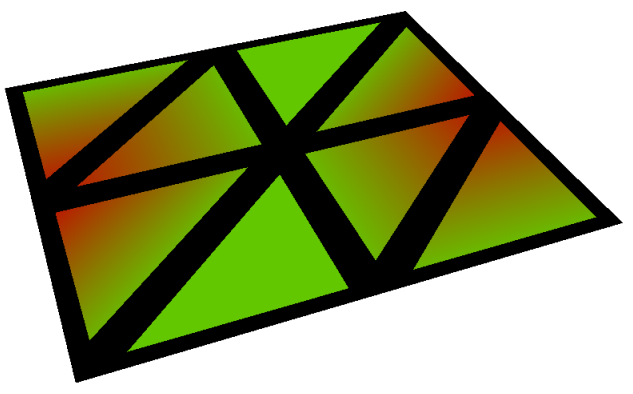

(b)8 faces

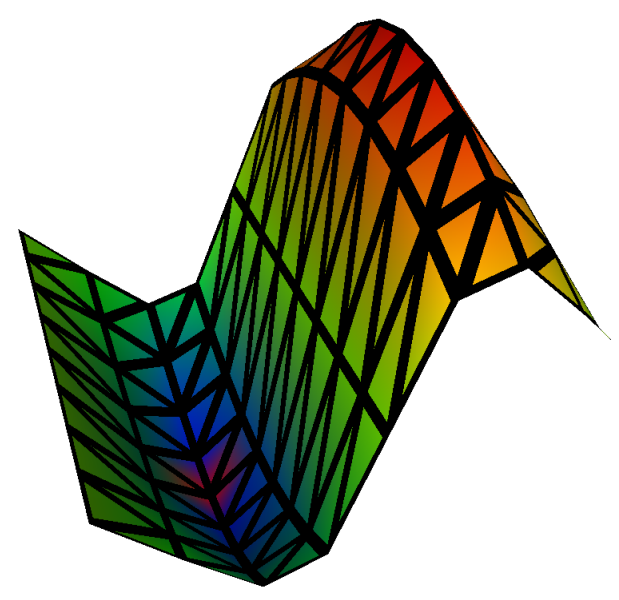

(d)128 faces

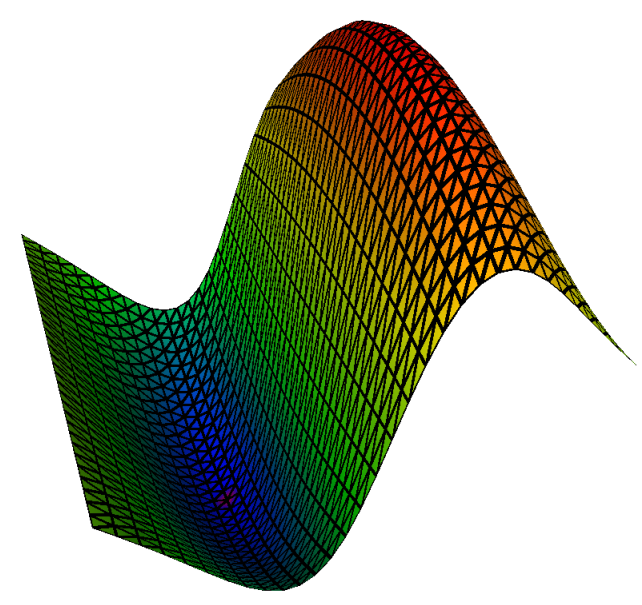

(f)8192 faces

Figura 6.2: Subdivisão de uma malha com dois triângulos para aproximar a função $z=\sin (\pi x) \cos y+0.1 \sin (2 \pi x)$ 


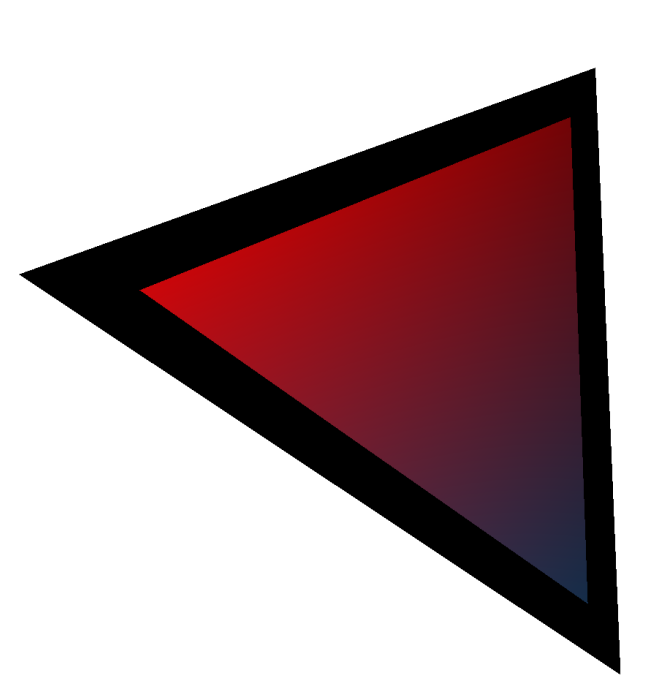

(a)4 faces

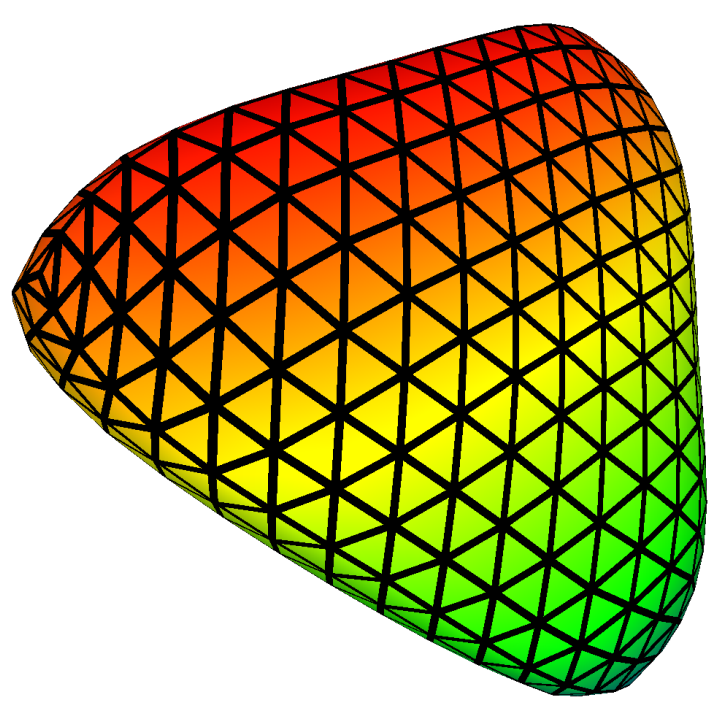

(c)1024 faces

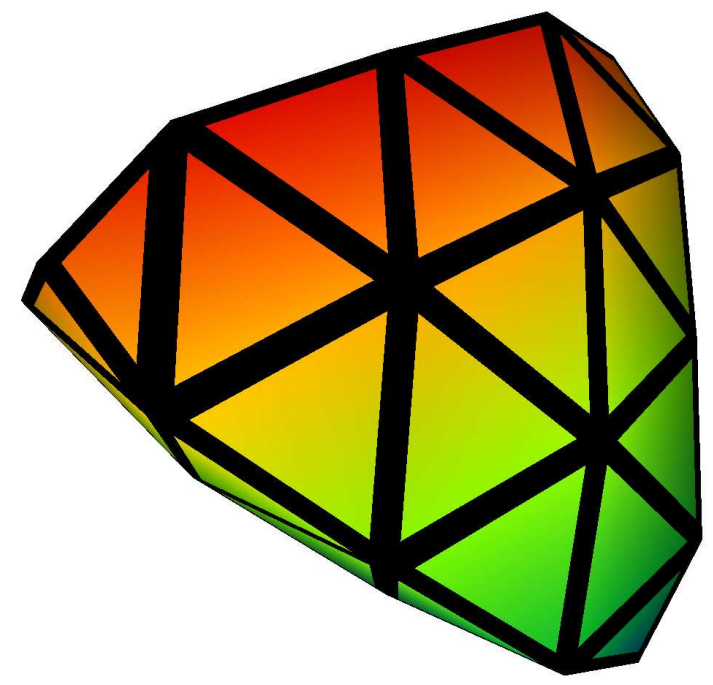

(b) 64 faces

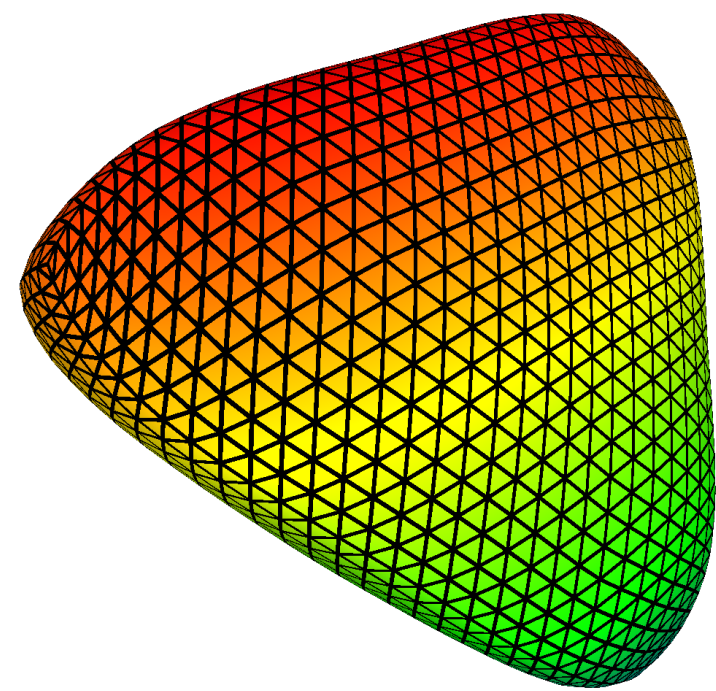

(d)4096 faces

Figura 6.3: Subdivisão de uma um tetraedro regular usando Butterfly 


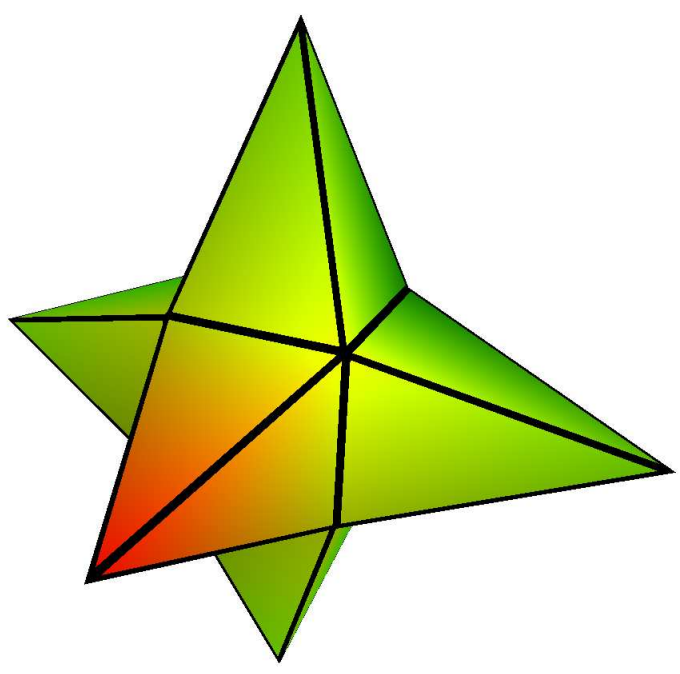

(a) 24 faces

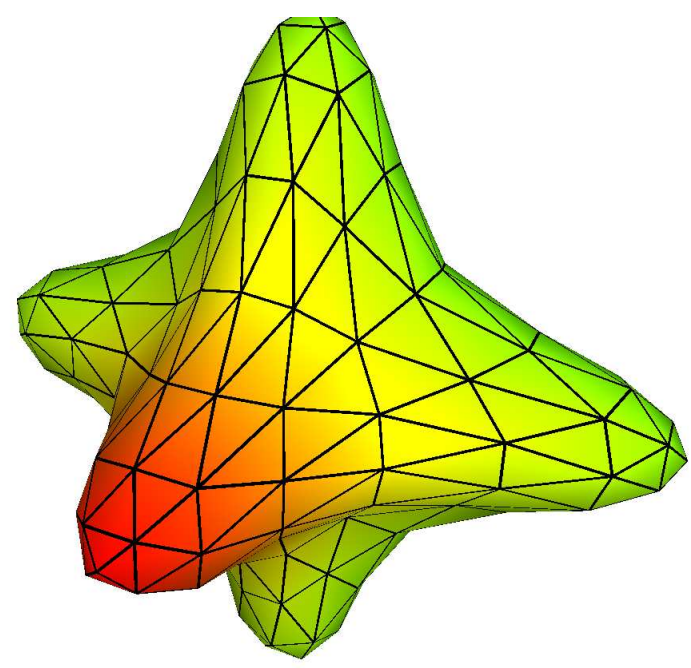

(c)384 faces

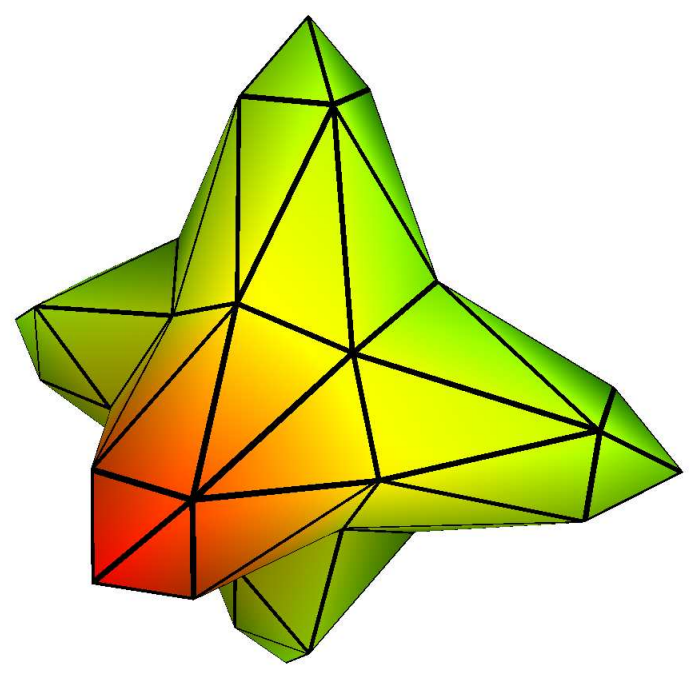

(b)96 faces

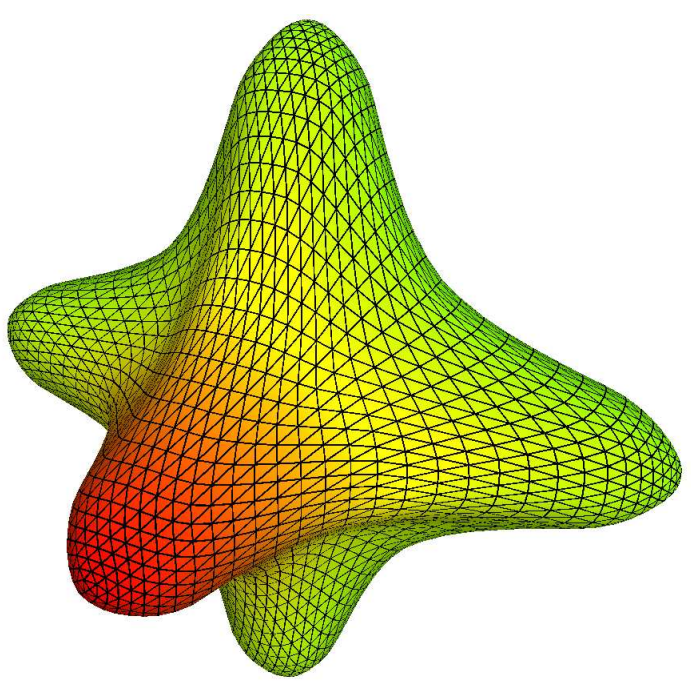

(d)6144 faces

Figura 6.4: Subdivisão de uma estrela de 6 pontas usando Butterfly 


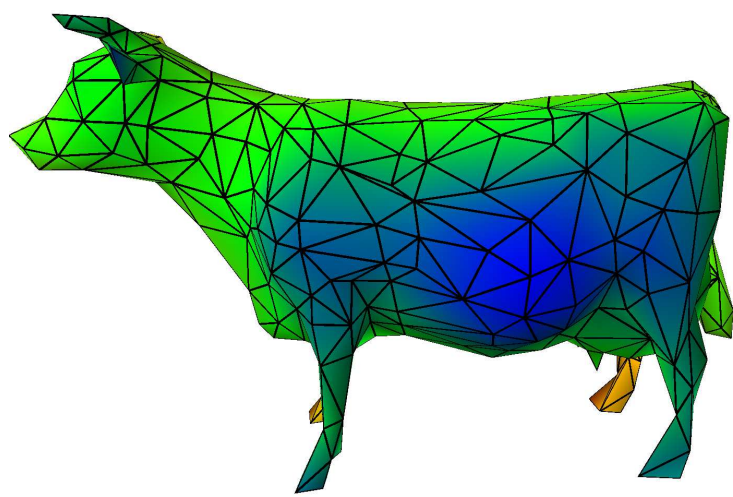

(a) 940 faces

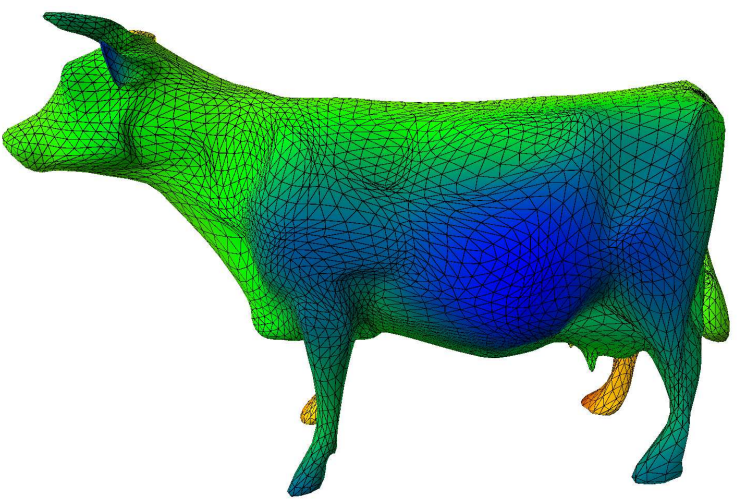

(c) 15040 faces

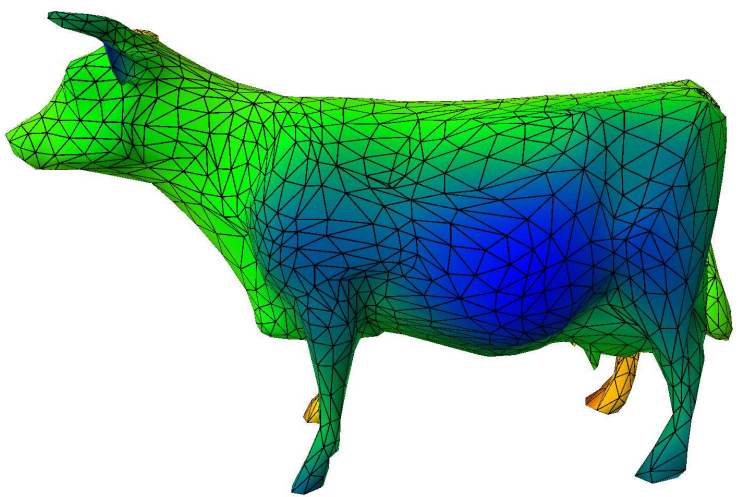

(b)3760 faces

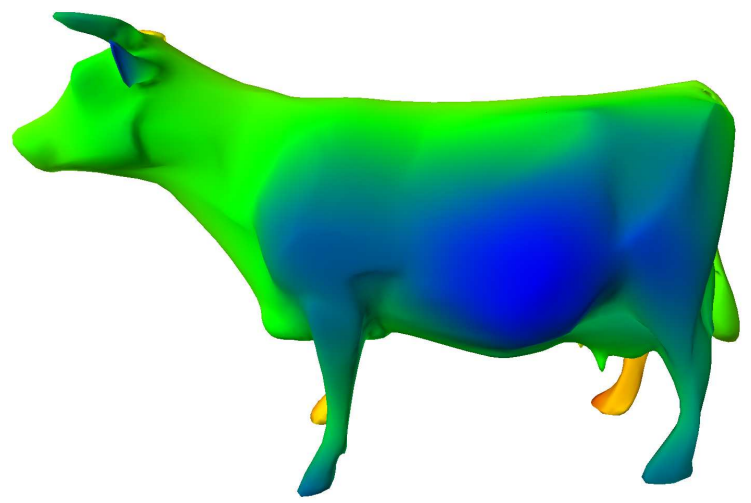

(d)Imagem final com 15040 faces

Figura 6.5: Subdivisão do modelo cow usando Butterfly. 


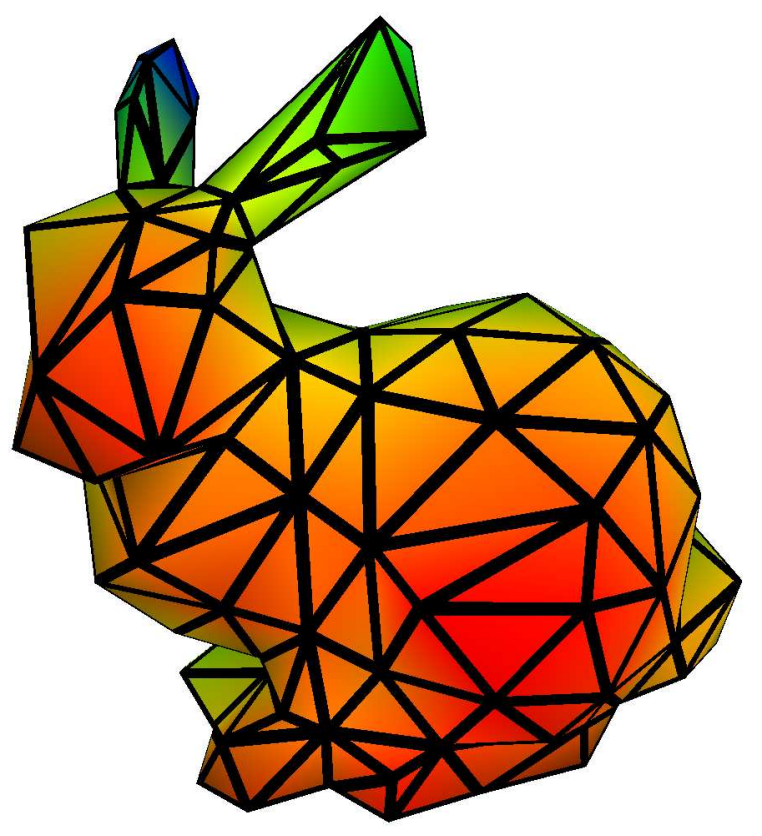

(a)340 faces

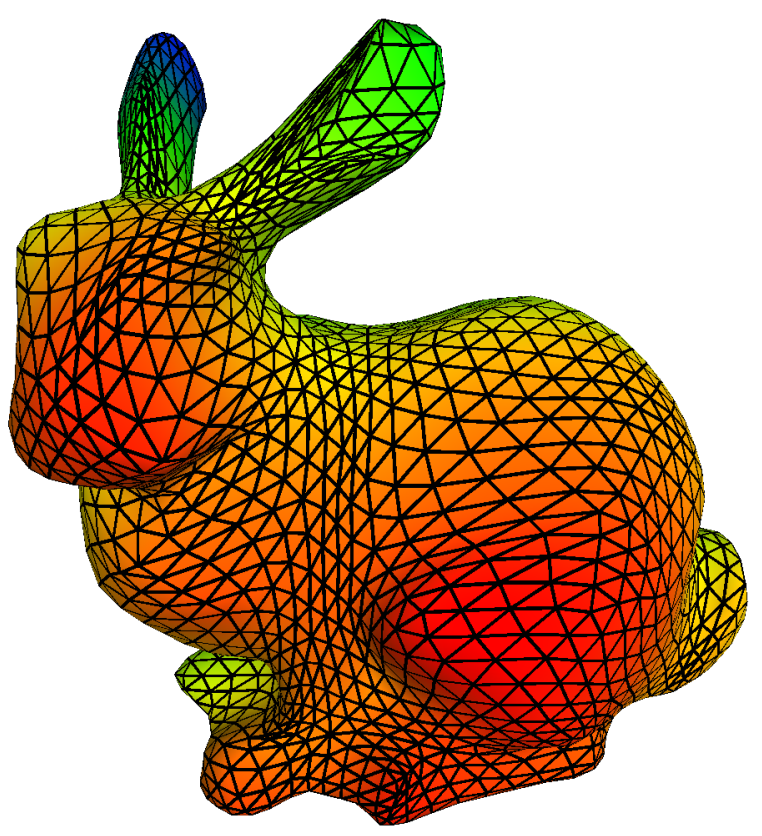

(c)5440 faces

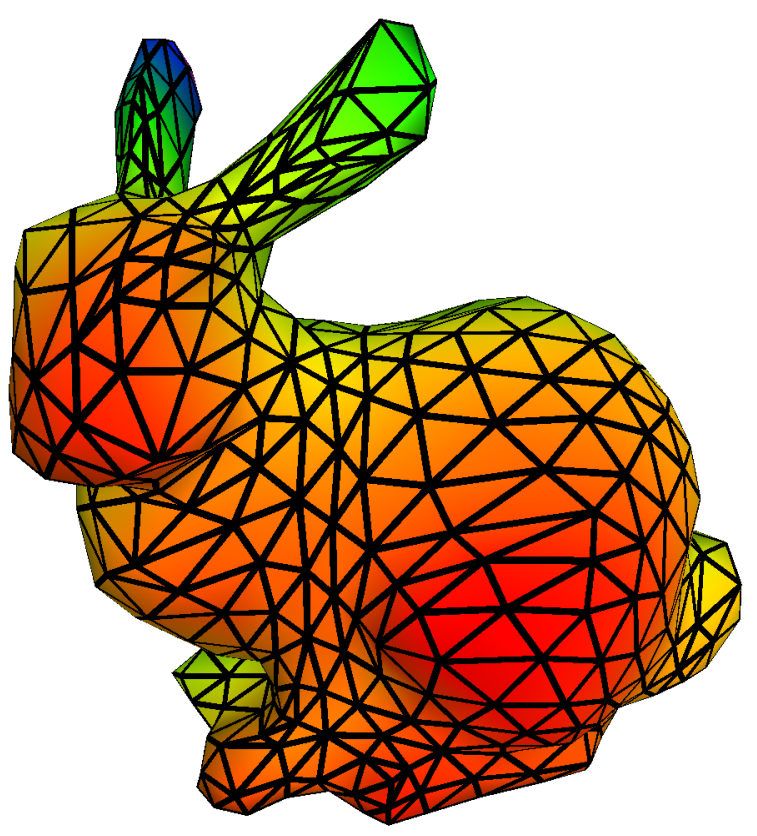

(b) 1360 faces

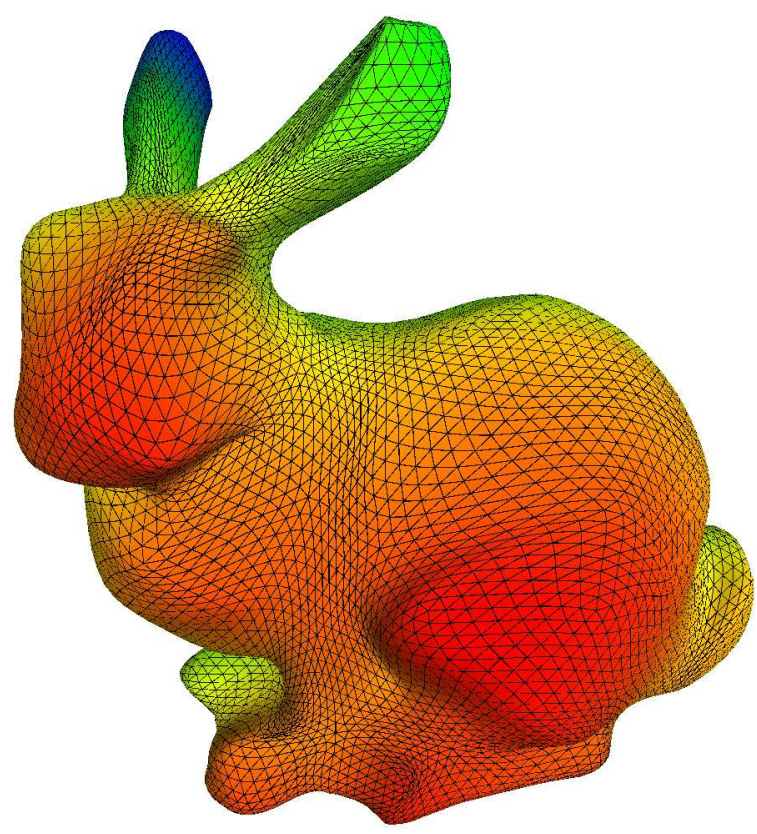

(d)21760 faces

Figura 6.6: Quatro níveis de subdivisão do modelo bunny usando Butterfly. 


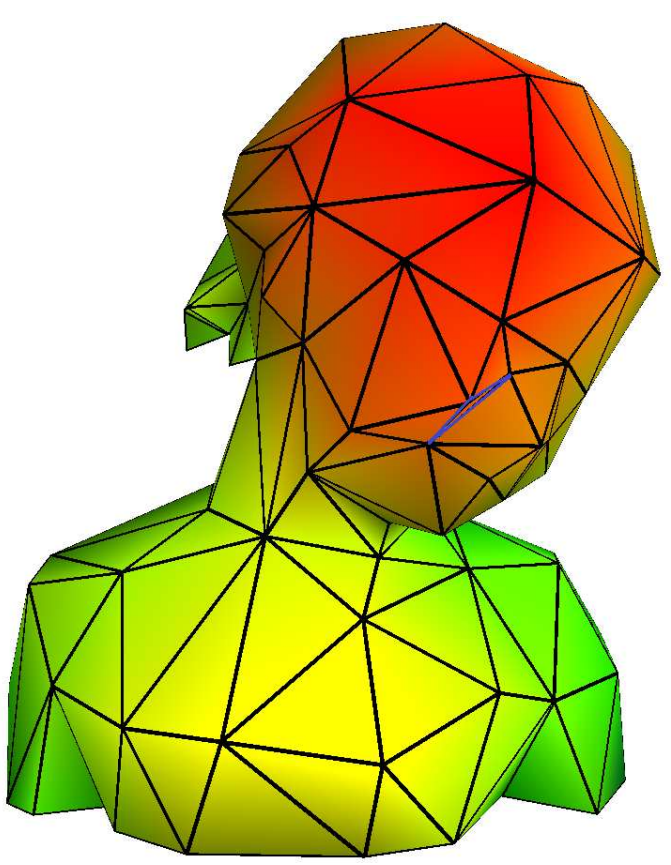

(a)262 faces

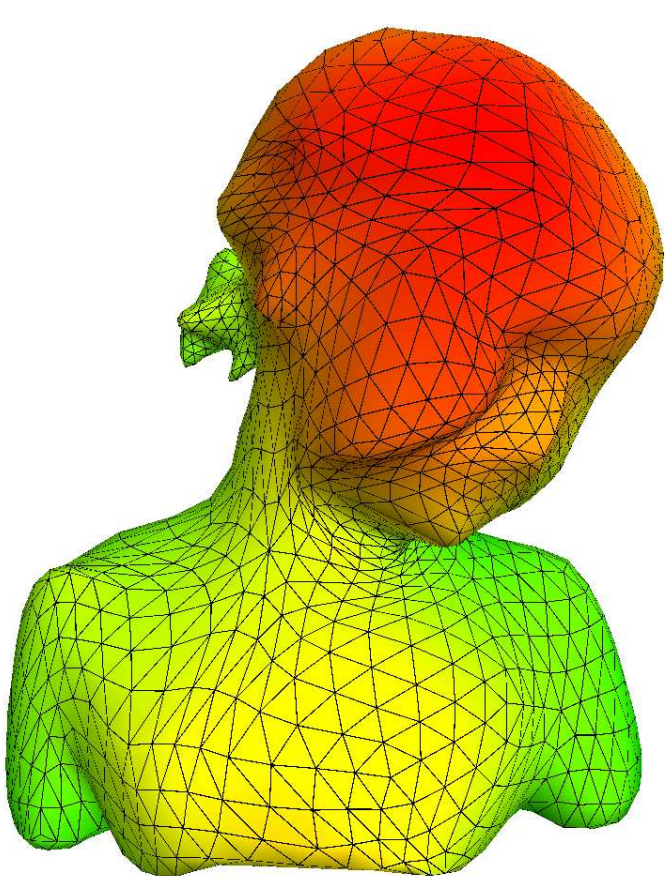

(c)4192 faces

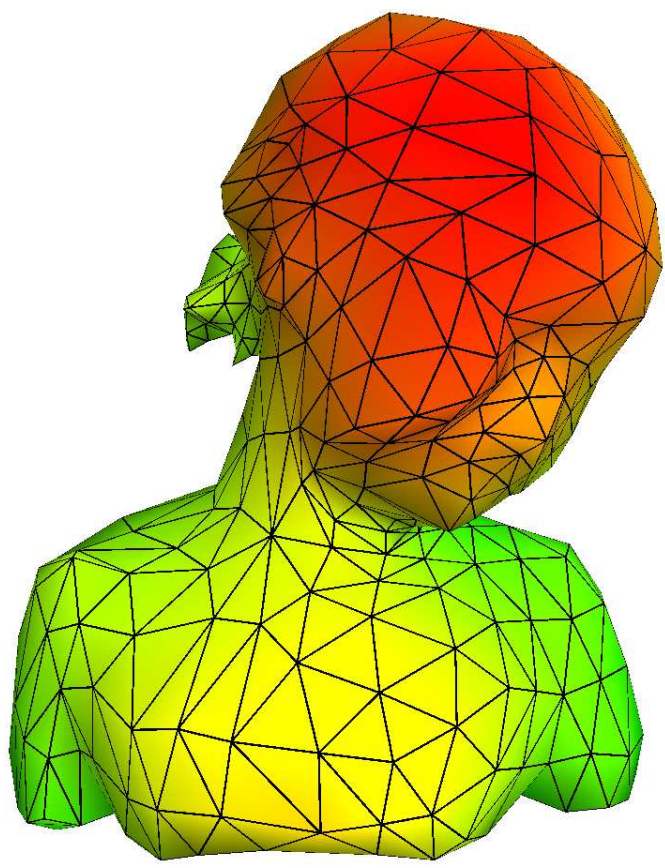

(b) 1048 faces

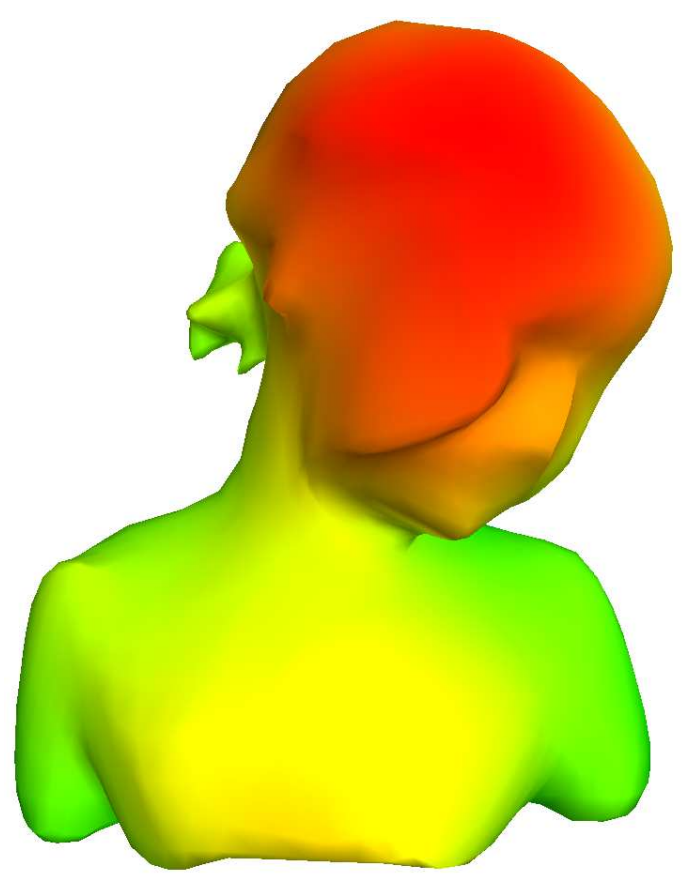

(d)Imagem final com 4192 faces

Figura 6.7: Subdivisão do modelo bimba usando Butterfly. O triângulo muito fino na em 6.7(a) provoca um efeito indesejado na subdivisão 


\section{2}

\section{Subdivisão adaptativa}

Como na subdivisão global, avaliamos a eficiência do algoritmo de subdivisão adaptativa aplicando-o recursivamente em uma malha triangular de uma superfície plana com bordo. Subdividimos recursivamente um triângulo onde os novos vértices criados pelo processo de subdivisão são sempre posicionados sobre o ponto médio das arestas.

\begin{tabular}{|c|c|c|c|c|c|}
\hline$|S|$ & $\left|E^{r}(S)\right|$ & $\begin{array}{c}\text { Faces } \\
\text { em } E^{r}(S)\end{array}$ & $\begin{array}{c}\text { Corners } \\
\text { s/ tab. } C\end{array}$ & $\begin{array}{c}\text { Corners } \\
\text { c/ tab. } C\end{array}$ & $\begin{array}{c}\text { Tem. } \\
\text { calc. } E^{r}(S)\end{array}$ \\
\hline 153 & 302 & 349 & $<0,01 s$ & $<0,01 s$ & $<0,01 s$ \\
\hline 561 & 1.034 & 1.213 & $0.01 s$ & $<0,01 s$ & $<0,01 s$ \\
\hline 2.145 & 3.794 & 4.477 & $0.03 s$ & $<0,01 s$ & $<0,01 s$ \\
\hline 8.385 & 14.498 & 17.149 & $0.19 s$ & $<0,01 s$ & $<0,01 s$ \\
\hline 33.153 & 56.642 & 67.069 & $2,52 s$ & $<0,01 s$ & $<0,01 s$ \\
\hline 131.841 & 223.874 & 265.213 & $37,22 s$ & $<0,01 s$ & $0.07 s$ \\
\hline 525.825 & 890.114 & 1.054 .717 & $\gg 37,22 s$ & $<0,01 s$ & $0.26 s$ \\
\hline 2.100 .225 & 3.549 .698 & 4.206 .589 & $\gg 37,22 s$ & $<0,01 s$ & $1.14 s$ \\
\hline 8.394 .753 & 14.177 .282 & 16.801 .789 & $\gg 37,22 s$ & $0,02 s$ & $4,78 s$ \\
\hline
\end{tabular}

Tabela 6.2: Dados da realização da subdivisão adaptativa com $r=1$. *Core i5 3.10Ghz, 8GB RAM

\begin{tabular}{|c|c|c|c|c|}
\hline$|S|$ & $\left|E^{r}(S)\right|$ & $\begin{array}{c}\text { Faces } \\
\text { em } E^{r}(S)\end{array}$ & $\begin{array}{c}\text { Tem. p/ } \\
\text { calc. faces }\end{array}$ & $\begin{array}{c}\text { Tem. p/ } \\
\text { sub. }\end{array}$ \\
\hline 153 & 302 & 349 & $<0,01 s$ & $<0,01 s$ \\
\hline 561 & 1.034 & 1.213 & $<0,01 s$ & $<0,01 s$ \\
\hline 2.145 & 3.794 & 4.477 & $0,01 s$ & $<0,01 s$ \\
\hline 8.385 & 14.498 & 17.149 & $0,01 s$ & $<0,01 s$ \\
\hline 33.153 & 56.642 & 67.069 & $0,05 s$ & $0,01 s$ \\
\hline 131.841 & 223.874 & 265.213 & $0,19 s$ & $0,12 s$ \\
\hline 525.825 & 890.114 & 1.054 .717 & $0,82 s$ & $0,36 s$ \\
\hline 2.100 .225 & 3.549 .698 & 4.206 .589 & $3,62 s$ & $1,5 s$ \\
\hline 8.394 .753 & 14.177 .282 & 16.801 .789 & $16,02 s$ & $7.3 s$ \\
\hline
\end{tabular}

Tabela 6.3: Dados da realização da subdivisão adaptativa com $r=1$. *Core i5 3.10Ghz, 8GB RAM

As tabelas 6.2 - 6.5 mostram o número de vértices do conjunto $S(|S|)$ da região selecionada para subdivisão, o número de vértices após realizar a expansão de $S$ para $E^{r}(S)\left(\left|E^{r}(S)\right|\right)$, o número de faces formadas pelos vértices do conjunto $E^{r}(S)$ que devem ser subdivididas e os tempos para realizar cada passo do algoritmo de subdivisão incremental: obter os corners dos vértices do conjunto $S$ usando uma Corner Table com e sem a tabela $C$, realizar a expansão de $S$ para $E^{r}(S)$, calcular as faces de $E^{r}(S)$ e por fim aplicar o algoritmo de subdivisão sobre 


\begin{tabular}{|c|c|c|c|c|c|}
\hline$|S|$ & $\left|E^{r}(S)\right|$ & $\begin{array}{c}\text { Faces } \\
\text { em } E^{r}(S)\end{array}$ & $\begin{array}{c}\text { Corners } \\
\text { s/ tab. } C\end{array}$ & $\begin{array}{c}\text { Corners } \\
\text { c/ tab. } C\end{array}$ & $\begin{array}{c}\text { Tem. } \\
\text { calc. } E^{r}(S)\end{array}$ \\
\hline 153 & 719 & 661 & $<0,01 s$ & $<0,01 s$ & $<0,01 s$ \\
\hline 561 & 2.224 & 1.909 & $0,01 s$ & $<0,01 s$ & $<0,01 s$ \\
\hline 2.145 & 7.362 & 5.941 & $0,04 s$ & $<0,01 s$ & $<0,01 s$ \\
\hline 8.385 & 26.286 & 20.149 & $0,3 s$ & $<0,01 s$ & $<0,01 s$ \\
\hline 33.153 & 98.809 & 73.141 & $3,71 s$ & $<0,01 s$ & $0.04 s$ \\
\hline 131.841 & 382.479 & 277.429 & $47,61 s$ & $<0,01 s$ & $0.14 s$ \\
\hline 525.825 & 1.504 .315 & 1.079 .221 & $\gg 37,22 s$ & $<0,01 s$ & $0,59 s$ \\
\hline 2.100 .225 & 5.965 .971 & 4.255 .669 & $\gg 37,22 s$ & $<0,01 s$ & $2,52 s$ \\
\hline 8.394 .753 & 23.761 .219 & 16.900 .021 & $\gg 37,22 s$ & $0,03 s$ & $10.55 s$ \\
\hline
\end{tabular}

Tabela 6.4: Dados da realização da subdivisão adaptativa com $r=5$. *Core i5 3.10Ghz, 8GB RAM

\begin{tabular}{|c|c|c|c|c|}
\hline$|S|$ & $\left|E^{r}(S)\right|$ & $\begin{array}{c}\text { Faces } \\
\text { em } E^{r}(S)\end{array}$ & $\begin{array}{c}\text { Tem. p/ } \\
\text { calc. faces }\end{array}$ & $\begin{array}{c}\text { Tem. p/ } \\
\text { sub. }\end{array}$ \\
\hline 153 & 719 & 661 & $<0,01 s$ & $<0,01 s$ \\
\hline 561 & 2.224 & 1.909 & $<0,01 s$ & $<0,01 s$ \\
\hline 2.145 & 7.362 & 5.941 & $0,01 s$ & $<0,01 s$ \\
\hline 8.385 & 26.286 & 20.149 & $0,02 s$ & $20,01 s$ \\
\hline 33.153 & 98.809 & 73.141 & $0,07 s$ & $0,02 s$ \\
\hline 131.841 & 382.479 & 277.429 & $0,32 s$ & $0,12 s$ \\
\hline 525.825 & 1.504 .315 & 1.079 .221 & $1,34 s$ & $0,38 s$ \\
\hline 2.100 .225 & 5.965 .971 & 4.255 .669 & $5,8 s$ & $1,59 s$ \\
\hline 8.394 .753 & 23.761 .219 & 16.900 .021 & $25.01 s$ & $7.26 s$ \\
\hline
\end{tabular}

Tabela 6.5: Dados da realização da subdivisão adaptativa com $r=5$. *Core i5 3.10Ghz, 8GB RAM

essas faces. As Tabelas 6.2 e 6.3 ilustram estes dados para um valor de $r=1$ e as Tabelas 6.4 e 6.5 para $r=5$.

Pela análise das tabelas é possível perceber que o passo que consome mais tempo é o cálculo das faces formadas por $E^{r}(S)$. Em seguida o cálculo da expansão do conjunto $S$ para o conjunto $E^{r}(S)$ e só então o passo de subdivisão, logo, para uma melhora na eficiência do algoritmo estes dois passos devem ser otimizados.

Como esperado, ao aumentar o valor de $r$ o número de vértices em $E^{r}(S)$ também aumenta, consequentemente aumentando o número de faces a serem subdivididas.

É importante notar que para aplicações que têm como entrada os vértices da região a ser subdividida a Corner Table com a tabela $C$ é essencial para realizar a subdivisão com eficiência, uma vez que as tabelas de tempos mostram um tempo muito alto para obter os corners dos vértices quando o conjunto $S$ atinge a ordem de 100 mil elementos. Em aplicações com modelos massivos, onde a lista de triângulos é muito grande, essa operação se torna muito cara. 
Executamos também a subdivisão adaptativa sobre um icosaedro regular para gerar a malha de uma esfera (Figura 6.8) aplicando vários níveis de subdivisão a partir de um conjunto de vértices usando $r=3$.

Na Figura 6.9, a subvisão adaptativa é usada aproximar uma malha plana da função $z=\sin (\pi x) \cos y+0.1 \sin (2 \pi x)$. Em todos os outrsos exemplos da subdivisão adaptativa, o esquema Butterfly foi utilizado o para posicionar os novos vértices.

Nas figuras $6.10,6.11,6.12,6.13$, um conjunto de vértices $S$ (em destaque) é selecionado para subdivisão e então vários níveis de subdivisão são aplicados sempre subdividindo o sonjunto $S^{\prime}$. Todos os resultados mostram uma transição suave entre a região selecionada para subdvisão e a região que não foi subdividida, além de uma boa qualidade dos triângulos resultantes, salvo os casos com a presença de triângulos muito finos na malha, como pode ser observado na Figura 6.11 . 


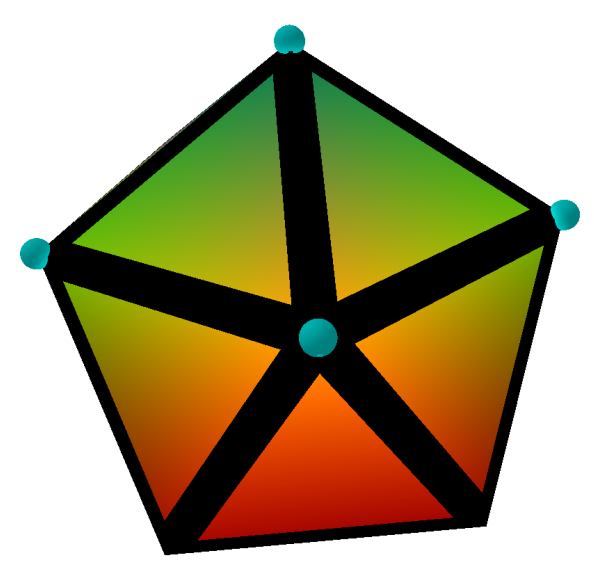

(a)Icosaedro regular com pontos selecionados para subdivisão. 20 faces

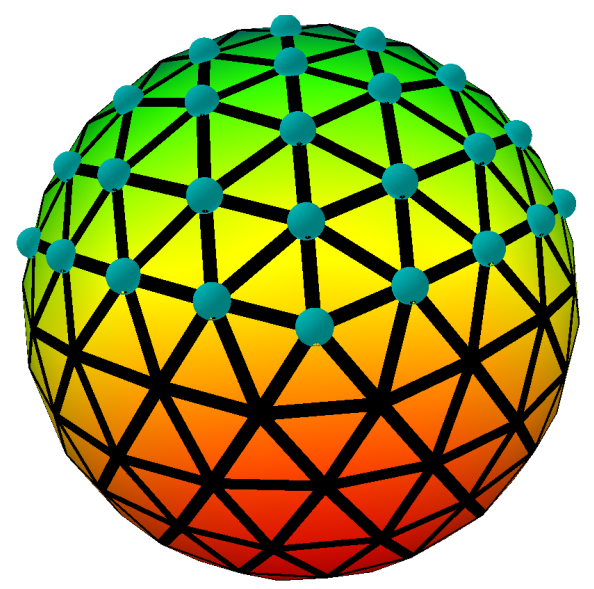

(b)2 leveis de subdivisão com $r=3.292$ faces

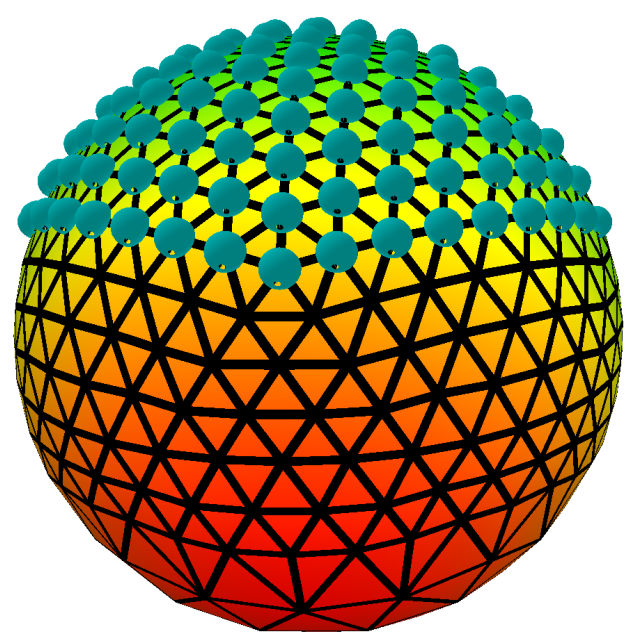

(c)4 leveis de subdivisão com $r=3.752$ faces

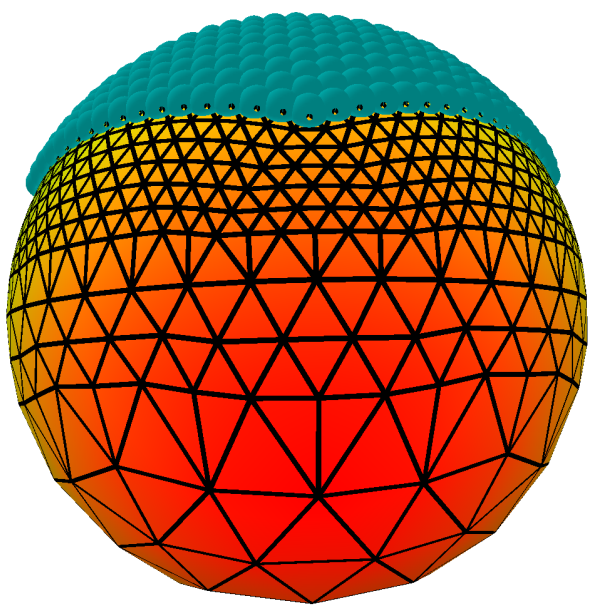

(e)5 leveis de subdivisão com $r=3.1084$ faces

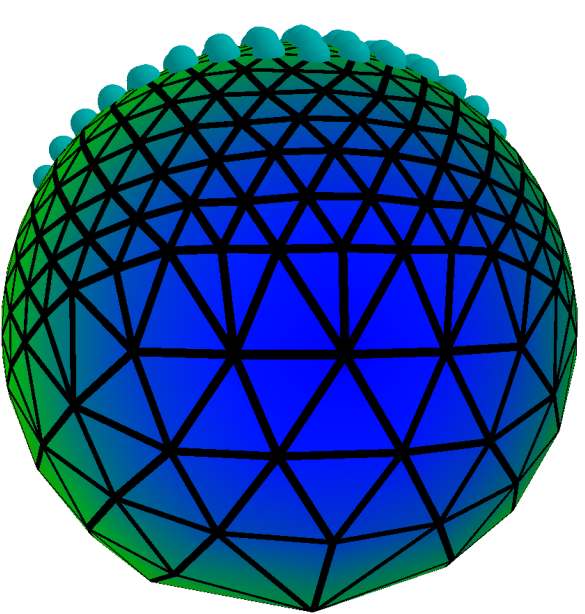

(d)Mesma esfera de c). Evidenciando a mudança de nível de subdivisão.

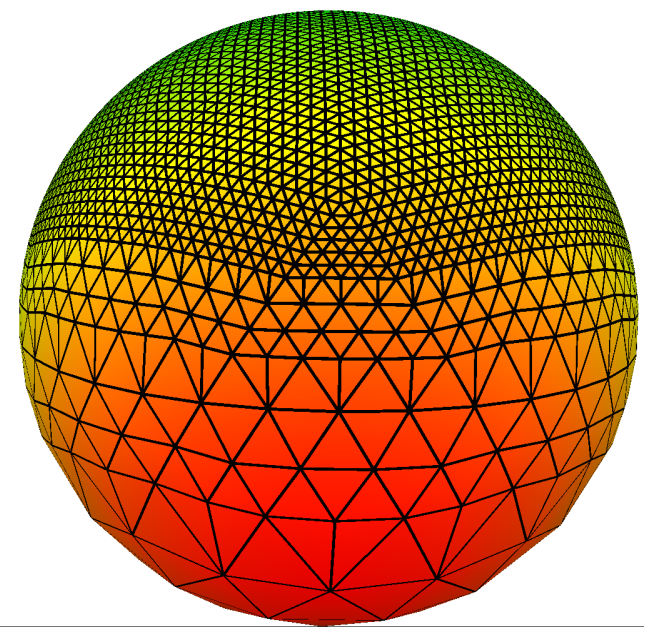

(f)6 leveis de subdivisão com $r=3.4016$ faces faces

Figura 6.8: Vários níveis da subdivisão incremental aplicada sobre um icosaedro regular para geração da malha de uma esfera. 


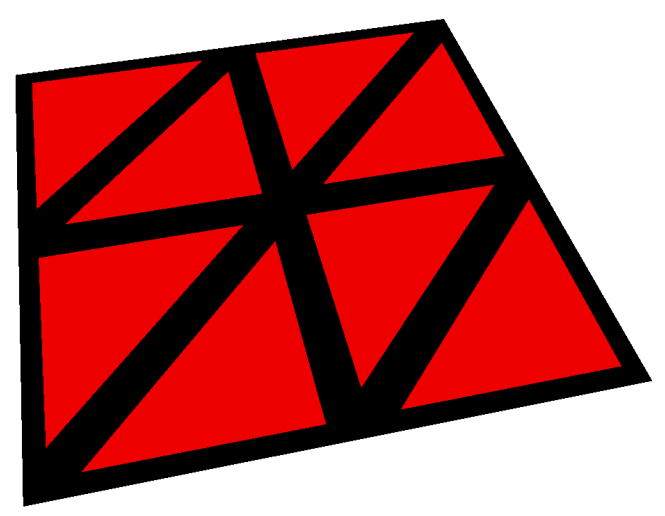

(a) 0 faces

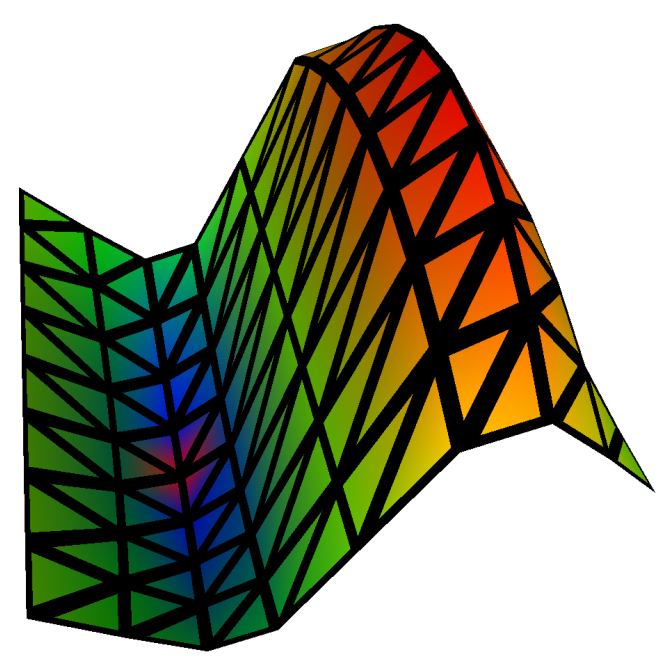

(c)32 faces

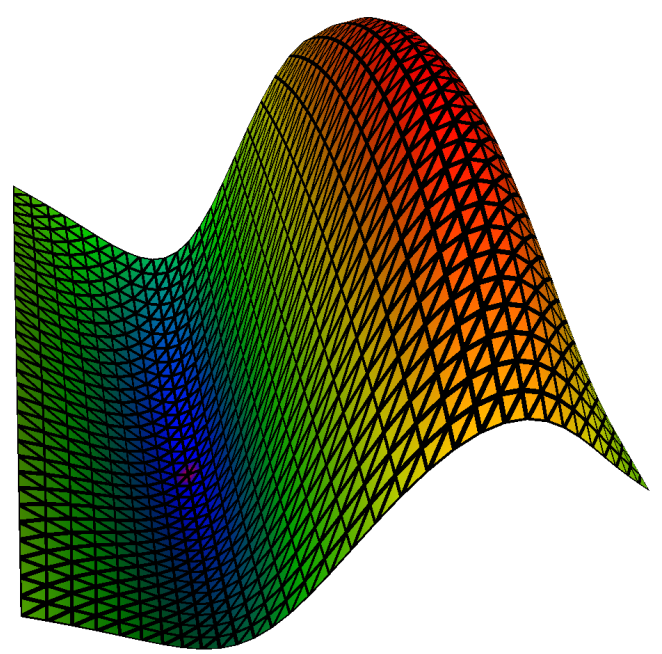

(e)2048 faces

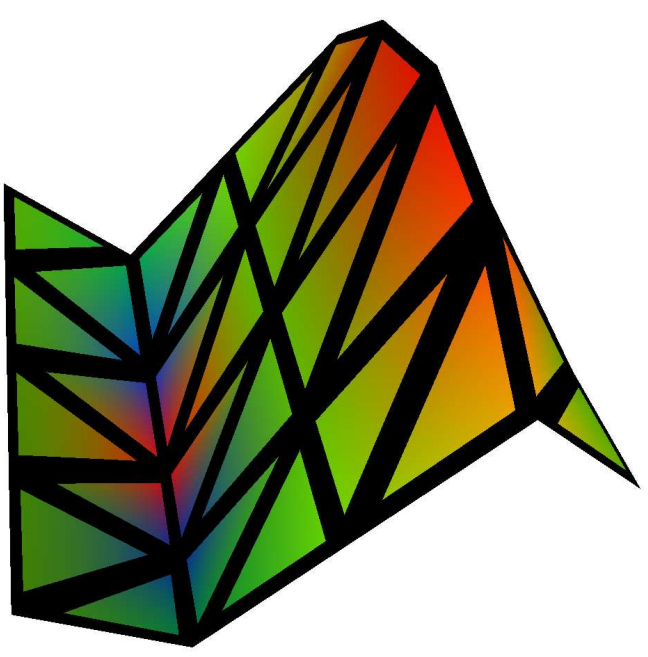

(b)8 faces

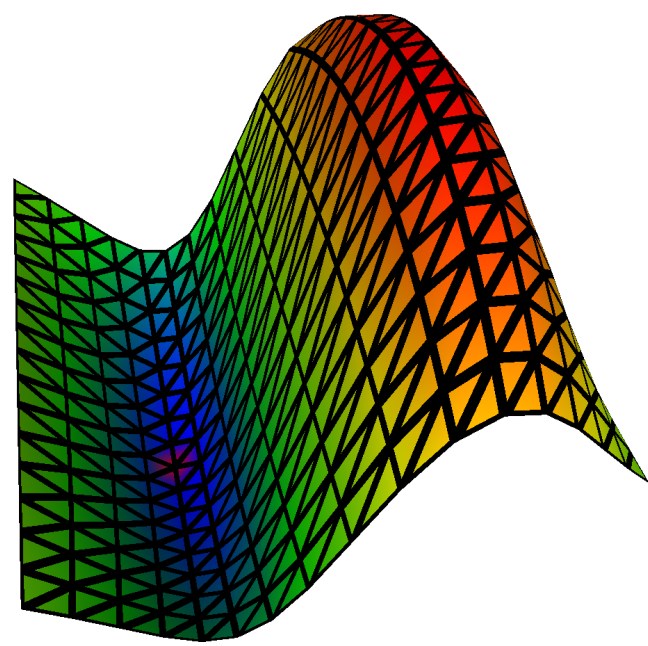

(d) 128 faces

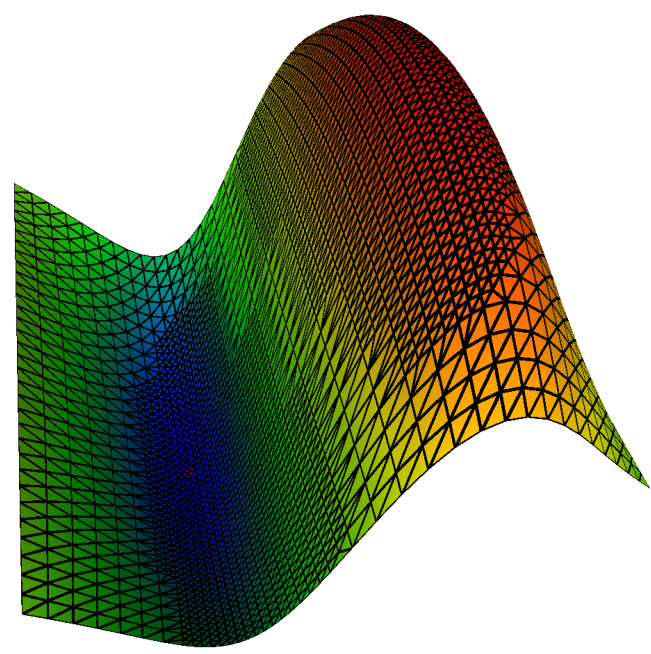

(f) 8192 faces

Figura 6.9: Subdivisão adaptativa de uma malha inicial com 8 triângulos para aproximar a função $z=\sin (\pi x) \cos y+0.1 \sin (2 \pi x)$. A cada passo a região onde o ponto médio de uma aresta dista mais que 0.005 da posição onde deveria está é selecionada para subdivisão 


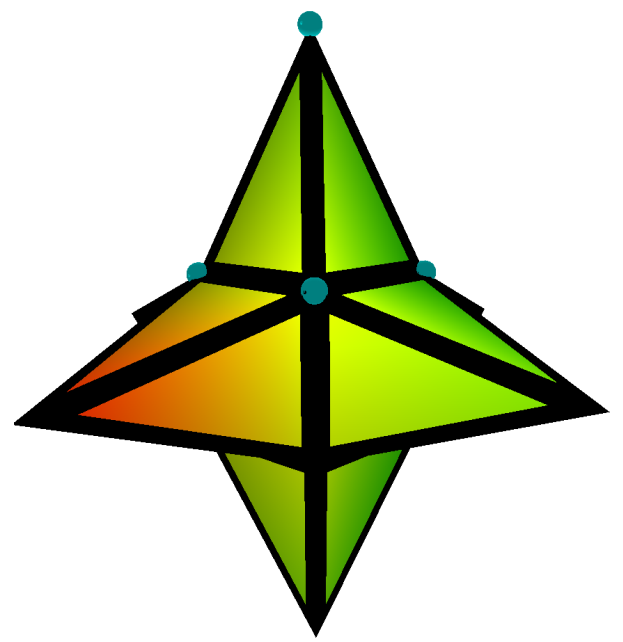

(a)Estrela de 6 pontos. 24 faces.

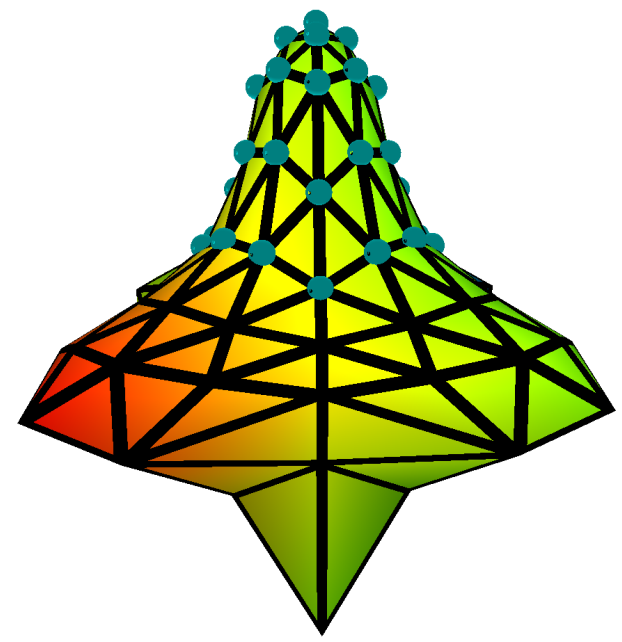

(c)2 níveis de subdivisão com $r=1.208$ faces.

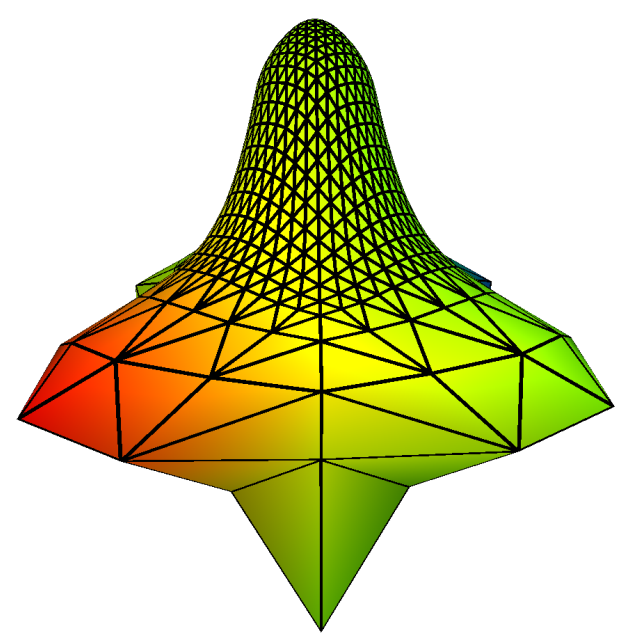

(e)4 níveis de subdivisão com $r=1.1536$ faces

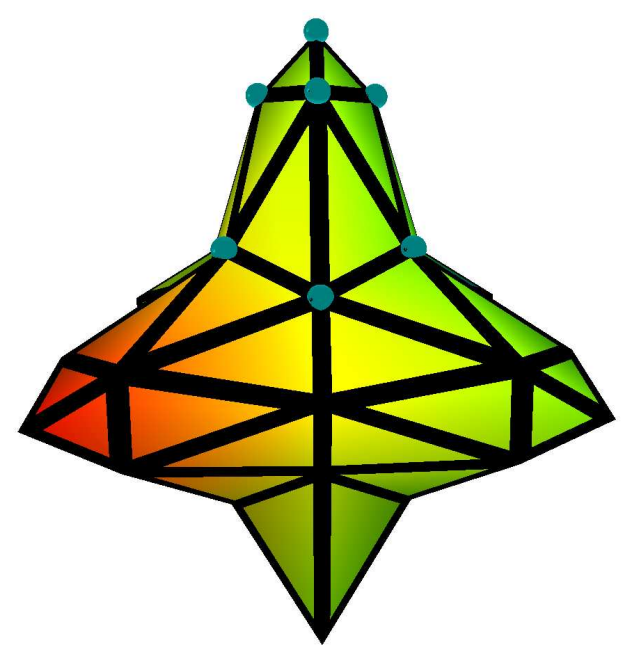

(b)1 nível de subdivisão com $r=1.88$ faces

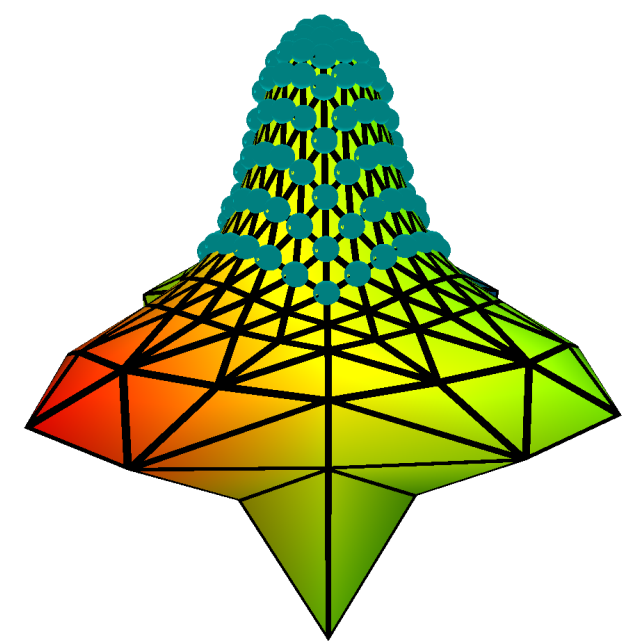

(d)3 níveis de subdivisão com $r=1.528$ faces

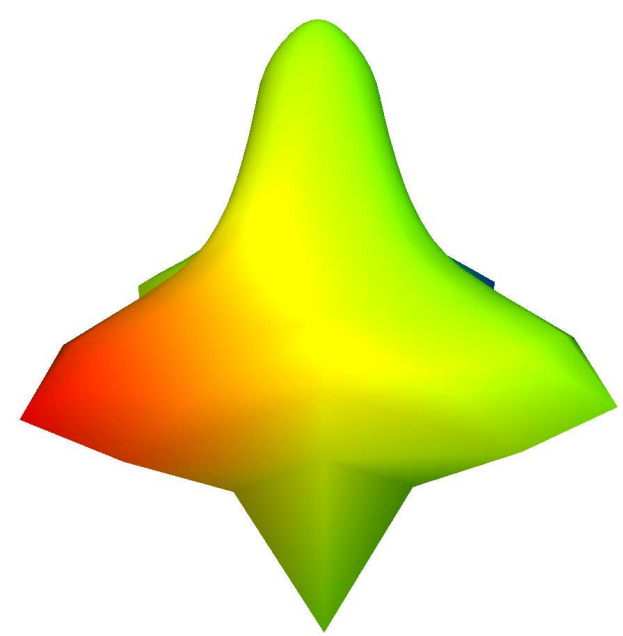

(f)Superfície final com 1536 faces faces

Figura 6.10: Vários níveis da subdivisão incremental aplicada sobre um icosaedro regular para geração da malha de uma esfera. 


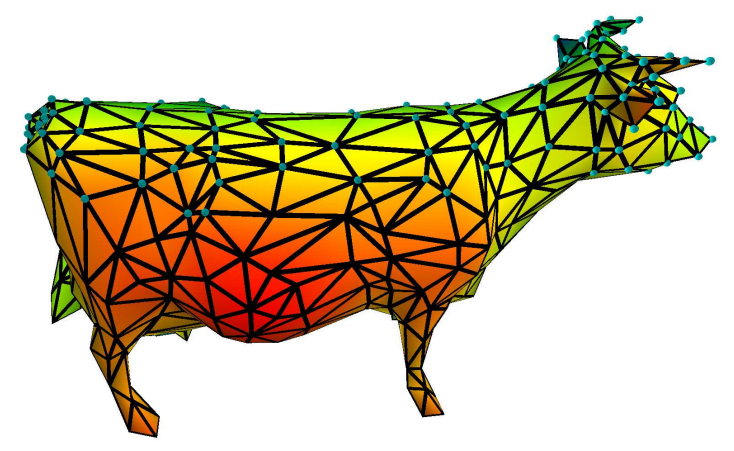

(a)Malha inicial do modelo cow. Os pontos selecionados são da região a ser subdividida, ou seja, o conjunto $S .940$ faces

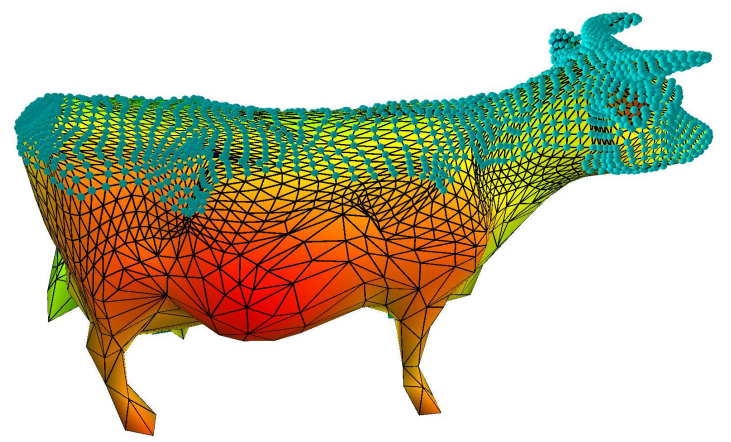

(c)2 níveis de subdivisão com $r=2.8206$ faces.

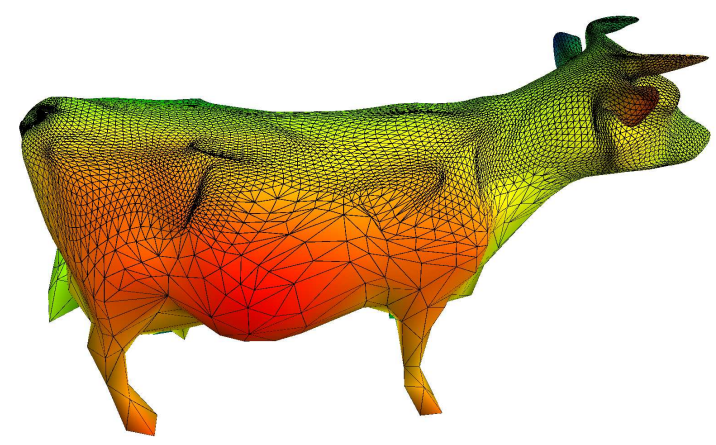

(e)Malha da superfície apresentada em c).

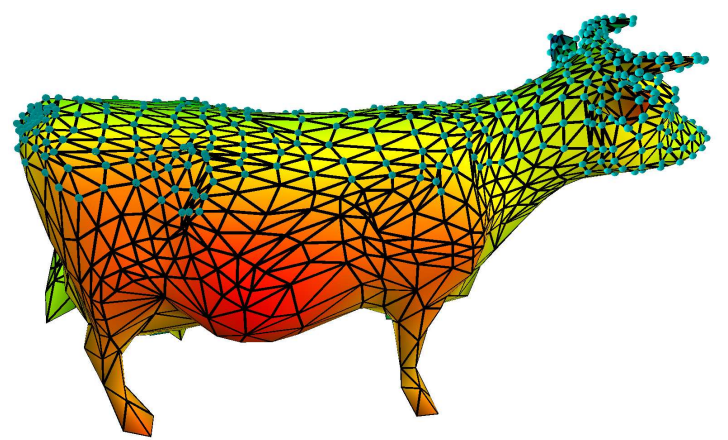

(b) 1 nível de subdivisão com $r=2.2628$ faces.

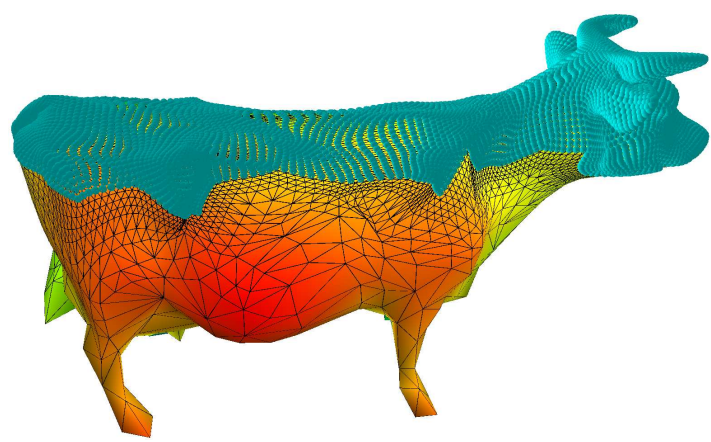

(d)3 níveis de subdivisão com $r=2.28122$ faces

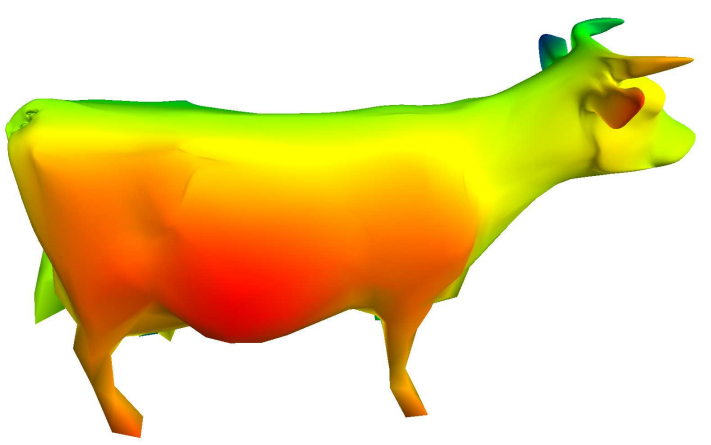

(f)Superfície final com 28122 faces.

Figura 6.11: Subdivisão incremental do modelo cow usando Butterfly para posicionar os novos vértices criados. Os pontos em destaque pertecem ao conjunto $S^{\prime}$, ou seja, o pontos a partir de onde o algoritmo faz a expansão. 


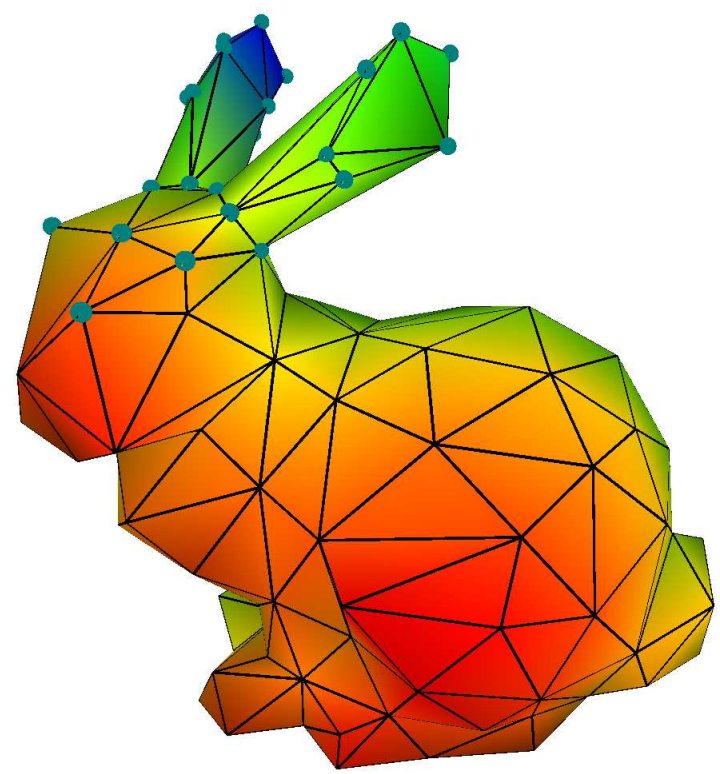

(a)Malha inicial do modelo bunny. Os pontos selecionados são da região a ser subdividida, ou seja, o conjunto $S .340$ faces

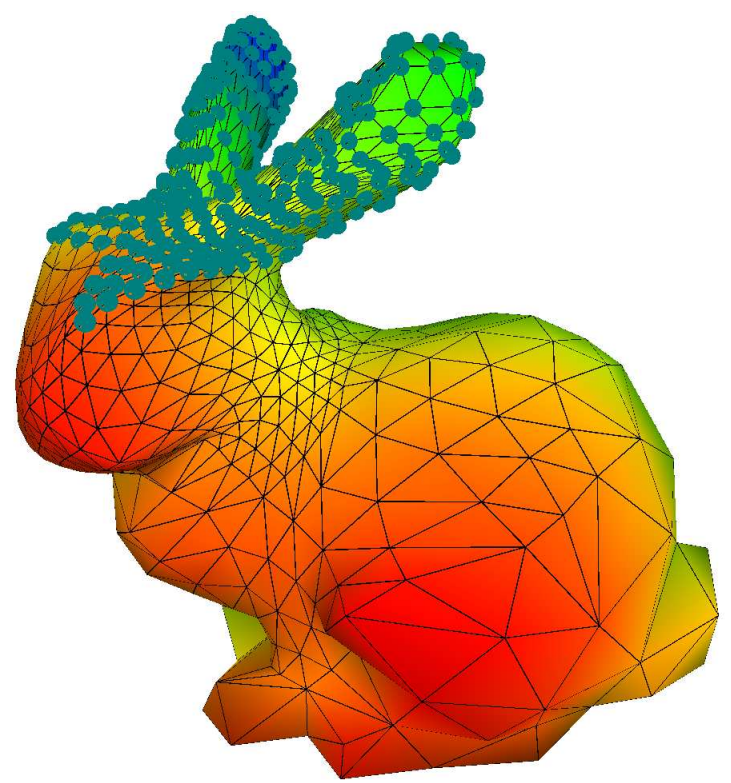

(c) 2 níveis de subdivisão com $r=4.1858$ faces.

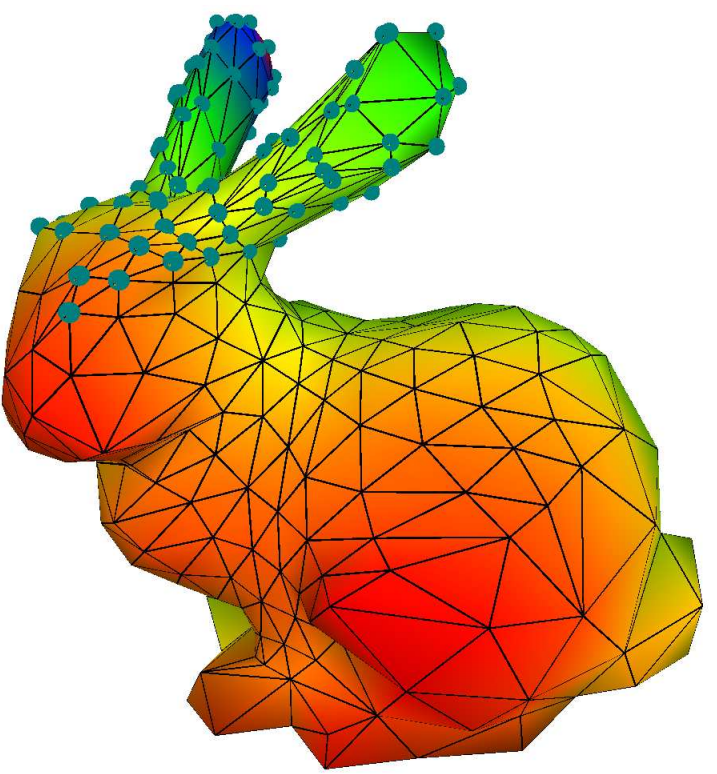

(b)1 nível de subdivisão com $r=4.800$ faces.

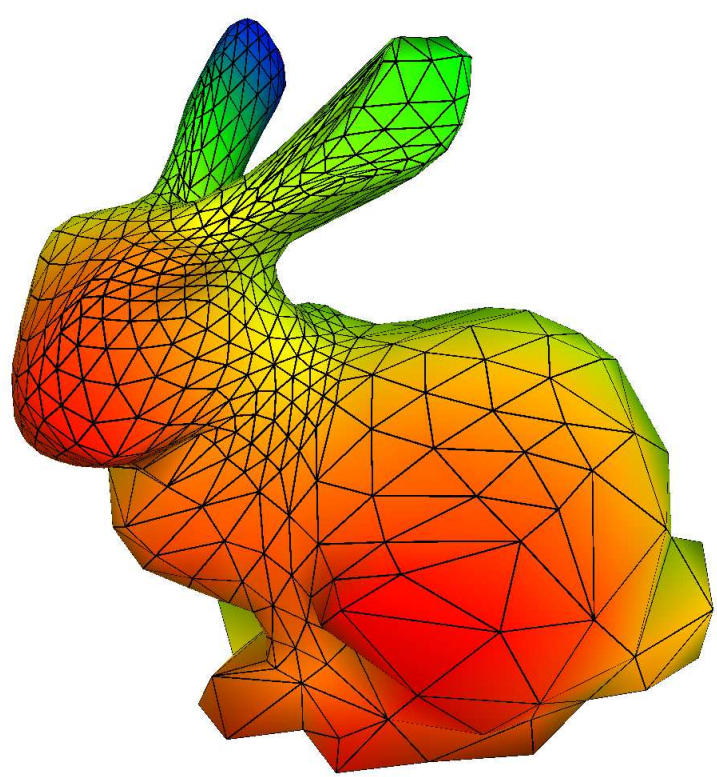

(d)Malha final com 1858 faces

Figura 6.12: Subdivisão incremental do modelo bunny usando Butterfly para posicionar os novos vértices criados. 


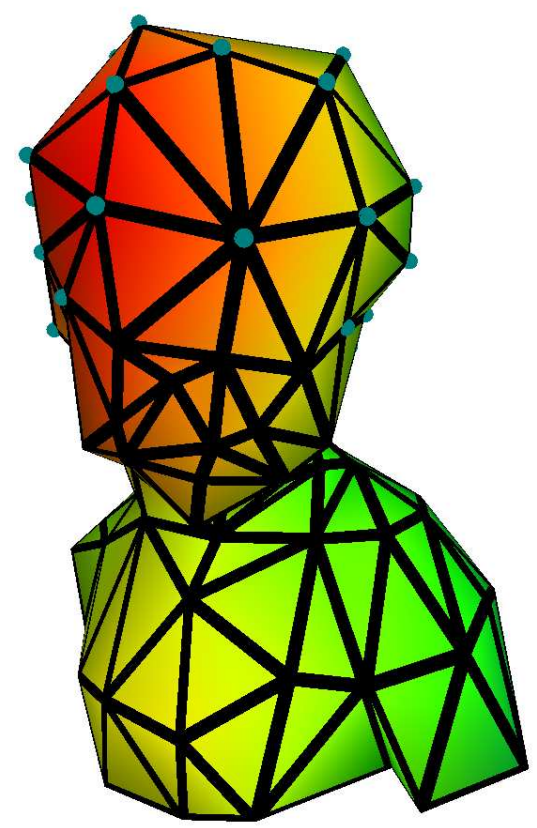

(a)Malha inicial do modelo bimba. Os pontos selecionados são da região a ser subdividida, ou seja, o conjunto $S .340$ faces

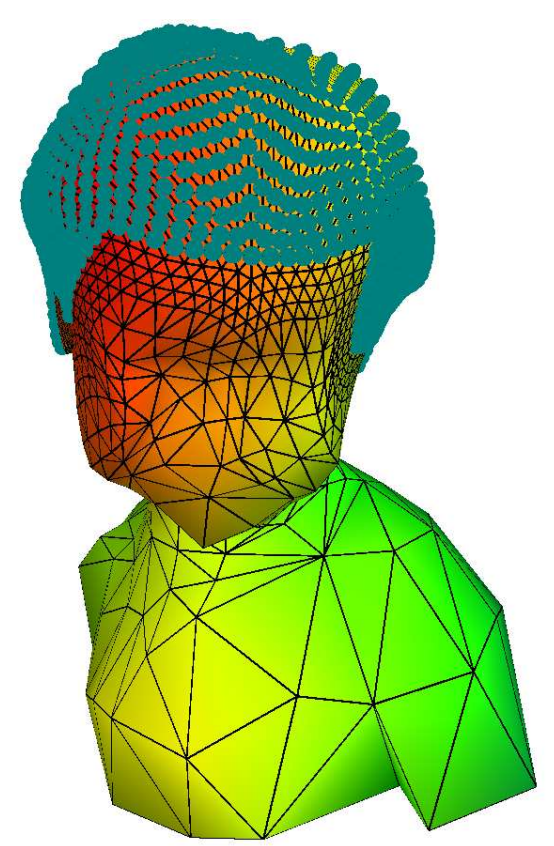

(c)3 níveis de subdivisão com $r=2.7288$ faces.



(b) 1 nível de subdivisão com $r=2.768$ faces.

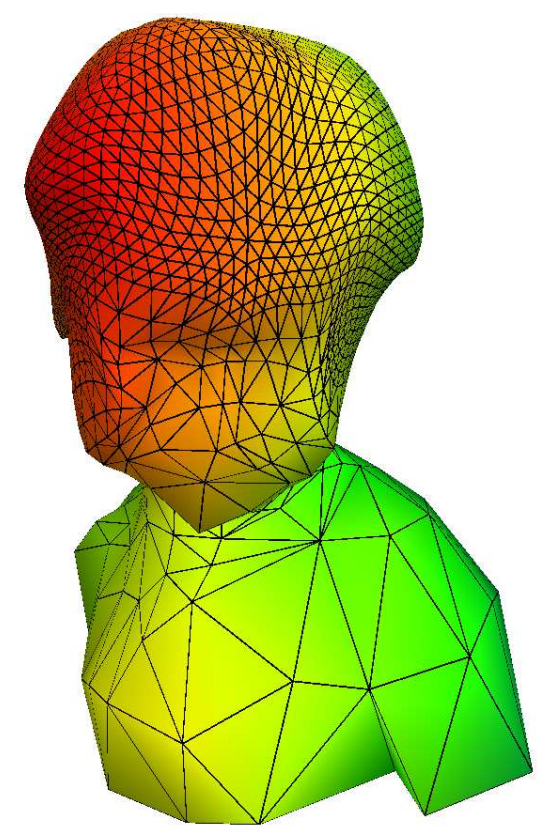

(d)Malha final com 7288 faces

Figura 6.13: Subdivisão incremental do modelo bimba usando Butterfly para posicionar os novos vértices criados. 


\section{7 \\ Conclusão e Trabalhos Futuros}

O objetivo de realizar subdivisão global e adaptativa de forma eficiente usando a Corner Table como estrutura de dados topológica de suporte foi alcançado. A implementação dos operadores estelares inversíveis preservaram a relação entre a triangulação e a estrutura de dados que a implementa, dando suporte a uma implementação simples e eficiente ao mecanismo de undo/redo. Essas ideias todas formam uma sólida base topológica para o desenvolvimento de algoritmos de refinamento e simplificação.

Para todos os testes, a Corner Table deu suporte eficiente à subdivisões global e adaptativa, exceto nos casos onde a região selecionada para a subdivisão adaptativa é definida pelos vértices. Nestes casos a tabela $C$ se faz necessária.

Os algoritmos propostos podem ser feitos independentemente da forma como a geometria dos novos vértices é calculada. No esquema Butterfly a qualidade da malha resultante depende da qualidade da malha inicial. De forma que malhas com triângulos distorcidos produzem dobras. Quando a geometria da superfície é conhecida, o refinamento se mostrou adequado no controle das transições.

Como trabalhos futuros sugerimos uma melhora nos algoritmos que efetuam a expansão do conjunto $S$ para $E^{r}(S)$ e calculam as faces em $E^{r}(S)$. 


\section{Referências Bibliográficas}

[Amresh et al, 2002] AMRESH, A.; FARIN, G. ; RAZDAN, A.. Adaptive subdivision schemes for triangular meshes. In: HIERARCHICAL AND GEOMETRIC METHODS IN SCIENTIFIC VISUALIZATION, p. 319327. Springer-Verlag, 2002. 2.2.1

[Andrew et al, 1983] ANDREW, R. B.; SHERMAN, A. H. ; WEISER, A.. Refinement algorithms and data structures for regular local mesh refinement. In: SCIENTIFIC COMPUTING, volumen 1, p. 3-17. IMACS/North-Holland, 1983. 2.2.1

[Baumgart, 1972] BAUMGART, B. G.. Winged edge polyhedron representation. Technical report, Stanford, CA, USA, 1972. 3

[Braid et al, 1980] BRAID, I. C.; HILLYARD, R. C. ; STROUD, I. A.. Stepwise construction of polyhedra in geometric modeling, p. 123-141. 1980. 3

[Cashman, 2012] CASHMAN, T. J.. Beyond catmull-clark? a survey of advances in subdivision surface methods. Comput. Graph. Forum, 31(1):42-61, Feb. 2012. 2

[Catmull and Clark, 1998] CATMULL, E.; CLARK, J.. Seminal graphics. chapter Recursively generated B-spline surfaces on arbitrary topological meshes, p. 183-188. ACM, New York, NY, USA, 1998. 2.1

[DeRose et al, 1998] DEROSE, T.; KASS, M. ; TRUONG, T.. Subdivision surfaces in character animation. In: PROCEEDINGS OF THE 25TH ANNUAL CONFERENCE ON COMPUTER GRAPHICS AND INTERACTIVE TECHNIQUES, SIGGRAPH '98, p. 85-94, New York, NY, USA, 1998. ACM. 2

[Doo and Sabin, 1978] DOO, D.; SABIN, M.. Behavior of recursive subdivision surfaces near extraordinary points. p. 356-360. ComputerAided Design, 1978. 2.1

[Dyn et al, 1990] DYN, N.; LEVINE, D. ; GREGORY, J. A.. A butterfly subdivision scheme for surface interpolation with tension control. ACM Trans. Graph., 9(2):160-169, Abril 1990. 2.1, 2.1.2 
[Dyn et al, 1993] DYN, N.; S, H. ; LEVIN, D.. Subdivision schemes for surface interpolation. Workshop in Computational Geomtry, 9(2):97-118, Abril 1993. 2.1.2

[Ferreira, 2006] DE OLIVEIRA LAGE FERREIRA, M.. Estruturas de dados topológicas escalonáveis para variedades de dimensão 2 e 3. Master's thesis, PUC Rio, Rio de Janeiro, Fevereiro 2006. (document), 3, 3.1.1, 3.1, 3.2, 3.7, 3.8, 3.9, 3.10, 3.2.3, 3.11, 5

[Jones et al, 1997] JONES, M. T.; PLASSMANN, P. E.. Parallel algorithms for adaptive mesh refinement. SIAM J. Sci. Comput., 18(3):686-708, May 1997. 2.2

[Kobblet, 2000] KOBBELT, L.. $\sqrt{3}$-subdivision. In: PROCEEDINGS OF THE 27TH ANNUAL CONFERENCE ON COMPUTER GRAPHICS AND INTERACTIVE TECHNIQUES, SIGGRAPH '00, p. 103-112, New York, NY, USA, 2000. ACM Press/Addison-Wesley Publishing Co. 2.2, 2.2

[Lickorish1999] LICKORISH, W. B. R.. Simplicial moves on complexes and manifolds. ArXiv Mathematics e-prints, Nov. 1999. 4.1

[Loop, 1987] LOOP, C.. Smooth subdivision surfaces based on triangles. Master's thesis, Department of Mathematics, University of Utah, Salt Lake City, August 1987. 2.1, 2.1.1, 2.1.1

[Mantyla, 1987] MÄNTYLÄ, M.. An introduction to solid modeling. Computer Science Press, Inc., New York, NY, USA, 1987. 3

[Newman, 1926] NEWMAN, M. H. A.. On the foundations of combinatorial analysis situs. In: PROC. ROYAL ACAD., p. 29:610-641, 1926. 4.1

[Pachner, 1991] PACHNER, U.. P1 homeomorphic manifolds are equivalent by elementary shellings. In: EUROP. J. COMBINATORICS, p. 12:129145, 1991. 4.1

[Pakdel and Samavati, 2007] PAKDEL, H.; SAMAVATI, F. F.. Incremental subdivision for triangle meshes. Int. J. Comput. Sci. Eng., 3(1):80-92, July 2007. (document), 2, 2.1, 2.1, 2.1.1, 2.1.1, 2.3, 2.1.2, 2.2, 2.2, 2.2.1, 2.2.1, 2.12, 2.2.2, 2.2.2, 2.15, 5.3

[Rossignac et al, 2001] ROSSIGNAC, J.; SAFONOVA, A. ; SZYMCZAK, A.. 3d compression made simple: Edgebreaker on a corner-table. In: SHAPE MODELING INTERNATIONAL CONFERENCE, p. 278-283, 2001. (document), 3, 3.1, 3.1.1, 3.3, 3.1.1 
[Velho and Zorin, 2001] VELHO, L.; ZORIN, D.. 4-8 subdivision. Comput. Aided Geom. Des., 18(5):397-427, June 2001. 2.2

[Velho, 2003] VELHO, L.. Dynamic adaptive meshes. In: IN DAGSTUHL SEMINAR ON HIERARCHICAL METHODS IN COMPUTER GRAPHICS, 2003. (document), 4.1, 4.1, 4.1

[Vieira, 2003] VIEIRA, A. W.. A topological approach for mesh simplification. Master's thesis, PUC Rio, Rio de Janeiro, Outubro 2003. (document), 3.1, 3.1.1, 3.2, 3.1.1, 3.3, 3.1.1, 3.1.2, 3.1.2, 2, 3.1.2, 3, 3.1.2, 4, 4.2, $6,4.5,9$

[Vieira et al, 2004] VIEIRA, A. W.; LEWINER, T.; VELHO, L.; LOPES, H. ; TAVARES, G.. Stellar mesh simplification using probabilistic optimization. Computer Graphics Forum, 23(4):825-838, october 2004. 1.1

[Zorin et al, 1996] ZORIN, D.; SCHRÖDER, P. ; SWELDENS, W.. Interpolating subdivision for meshes with arbitrary topology. In: PROCEEDINGS OF THE 23RD ANNUAL CONFERENCE ON COMPUTER GRAPHICS AND INTERACTIVE TECHNIQUES, SIGGRAPH '96, p. 189-192, New York, NY, USA, 1996. ACM. (document), 2, 2.1.2, 2.1.2, 2.9

[Zorin at al, 1997] ZORIN, D.; SCHRÖDER, P. ; SWELDENS, W.. Interactive multiresolution mesh editing. In: PROCEEDINGS OF THE 24TH ANNUAL CONFERENCE ON COMPUTER GRAPHICS AND INTERACTIVE TECHNIQUES, SIGGRAPH '97, p. 259-268, New York, NY, USA, 1997. ACM Press/Addison-Wesley Publishing Co. 2.2.1 